\title{
Highly Selective and Stable Isolated Non-Noble Metal Atom \\ Catalysts for Selective Hydrogenation of Acetylene
}

Baoai Fu, ${ }^{1}$ Alan J. McCue, ${ }^{2}$ Yanan Liu, ${ }^{1, *}$ Shaoxia Weng, ${ }^{1}$ Yuanfei Song, ${ }^{1}$ Yufei He, ${ }^{1}$ Junting

$$
\text { Feng }{ }^{1} \text { and Dianqing } \mathrm{Li}^{1, *}
$$

${ }^{1}$ State Key Laboratory of Chemical Resource Engineering, Beijing Engineering Center for

Hierarchical Catalysts, Beijing University of Chemical Technology, Beijing, 100029, China

${ }^{2}$ Department of Chemistry, University of Aberdeen, Aberdeen AB24 3UE, U.K.

\author{
* Corresponding author \\ Address: Box 98, 15 Bei San Huan East Road, Beijing 100029, China \\ Tel: +861064436992 Fax: +861064436992 \\ E-mail address: ynliu@mail.buct.edu.cn (Yanan Liu); lidq@mail.buct.edu.cn (Dianqing Li)
}

\section{Experimental Section}

Materials. $\mathrm{Ni}\left(\mathrm{NO}_{3}\right)_{2} \cdot 6 \mathrm{H}_{2} \mathrm{O},\left(\mathrm{NH}_{4}\right)_{2} \mathrm{MoS}_{4}, \mathrm{NaBH}_{4}, \mathrm{HCl}$ and $\mathrm{Al}_{2} \mathrm{O}_{3}$ were A.R. grade and utilized without any purification. Deionized water was self-prepared with a conductivity of $<10^{-6} \mathrm{~S} / \mathrm{cm}$.

Preparation of $\left[\mathrm{Moz}_{4}\left(\mathrm{H}_{2} \mathrm{O}\right){ }_{9}\right]^{4+}$ precursor. $1.12 \mathrm{~g}$ of $\mathrm{NaBH}_{4}$ (dissolved in $15 \mathrm{~mL}$ of $\left.\mathrm{H}_{2} \mathrm{O}\right)$ and $6 \mathrm{~mol} / \mathrm{L} \mathrm{HC1}(15 \mathrm{~mL})$ were alternatively pipetted into $35 \mathrm{~mL} 110 \mathrm{mmol} / \mathrm{L}$ of $\left(\mathrm{NH}_{4}\right)_{2} \mathrm{MoS}_{4}$ solution under vigorous stirring at room temperature. Then, $60 \mathrm{~mL} 6 \mathrm{~mol} / \mathrm{L}$ $\mathrm{HCl}$ was added to the above obtained suspension at a temperature of $90^{\circ} \mathrm{C}$ for $12 \mathrm{~h}$. During air oxidation, the suspension was transformed into a solution with dark green color. Once the volume of the solution decreased below $100 \mathrm{~mL}$, a further portion of 1 $\mathrm{mol} / \mathrm{L} \mathrm{HCl}$ was added to maintain acidic conditions. Subsequently, $0.37 \mathrm{~g}$ of $\mathrm{Ni}\left(\mathrm{NO}_{3}\right)_{2} \cdot 6 \mathrm{H}_{2} \mathrm{O}$ was dissolved in $10 \mathrm{~mL}$ of deionized water and then mixed with $5 \mathrm{~mL}$ of the $\left[\mathrm{Mo}_{3} \mathrm{~S}_{4}\left(\mathrm{H}_{2} \mathrm{O}\right)_{9}\right]^{4+}$ solution to produce $\left[\mathrm{Mo}_{3} \mathrm{NiS}_{4}\left(\mathrm{H}_{2} \mathrm{O}\right)_{10}\right]^{4+}$ precursor.

Preparation of a series of $\mathrm{Ni}$ and $\mathrm{Mo-based}$ catalysts. $\mathrm{NiMoS} / \mathrm{Al}_{2} \mathrm{O}_{3}$ catalysts with different Ni loadings of 0.6 wt.\%, 2.5 wt. $\%$ and 3.5 wt.\% were prepared. In detail, 1.5 $\mathrm{g}$ of $\mathrm{Al}_{2} \mathrm{O}_{3}$ power or sphere was added into an appropriate volume of 
$\left[\mathrm{Mo}_{3} \mathrm{NiS}_{4}\left(\mathrm{H}_{2} \mathrm{O}\right)_{10}\right]^{4+}$ solution under stirring at $50^{\circ} \mathrm{C}$ for $4 \mathrm{~h}$, followed by drying at $60^{\circ} \mathrm{C}$ for $12 \mathrm{~h}$. The obtained sample was treated at $200^{\circ} \mathrm{C}$ in $\mathrm{N}_{2}$ for $1 \mathrm{~h}$ and then at $300^{\circ} \mathrm{C}$ in $\mathrm{H}_{2}$ for $2 \mathrm{~h}$ to obtain $\mathrm{Ni}_{1} \mathrm{MoS} / \mathrm{Al}_{2} \mathrm{O}_{3}$ or $\mathrm{Ni}_{1} \mathrm{MoS} / \mathrm{S}-\mathrm{Al}_{2} \mathrm{O}_{3}$ catalyst. Other samples were prepared in a similar manner but using $\left[\mathrm{Mo}_{3} \mathrm{~S}_{4}\left(\mathrm{H}_{2} \mathrm{O}\right)_{9}\right]^{4+}, \mathrm{Ni}\left(\mathrm{NO}_{3}\right)_{2} \cdot 6 \mathrm{H}_{2} \mathrm{O}$ and $\left(\mathrm{NH}_{4}\right)_{2} \mathrm{MoO}_{4}$ salts as the metal precursors. Following the same thermal treatment described above, $\mathrm{MoS} / \mathrm{Al}_{2} \mathrm{O}_{3}, \mathrm{Ni} / \mathrm{Al}_{2} \mathrm{O}_{3}$ and $\mathrm{Mo} / \mathrm{Al}_{2} \mathrm{O}_{3}$ samples were prepared for contrast. To prepare $\mathrm{NiS} / \mathrm{Al}_{2} \mathrm{O}_{3}$ catalyst, appropriate amounts of $\mathrm{Ni}\left(\mathrm{NO}_{3}\right)_{2} \cdot 6 \mathrm{H}_{2} \mathrm{O}$ and $\mathrm{Na}_{2} \mathrm{~S} \cdot 9 \mathrm{H}_{2} \mathrm{O}$ metal salts were dissolved in $10 \mathrm{~mL}$ deionized water, before $1.5 \mathrm{~g}$ of $\mathrm{Al}_{2} \mathrm{O}_{3}$ support was added and stirred for $4 \mathrm{~h}$. The resulting suspension was dried at $50^{\circ} \mathrm{C}$ for $12 \mathrm{~h}$, and activated using the same treatment conditions described above.

Characterization. Powder X-Ray Diffraction (XRD) data were recorded from 10 to $80^{\circ}$ employing a diffractometer (Shimadzu XRD-6000) operated with $\mathrm{Cu} \mathrm{K} \alpha$ radiation $(\lambda=0.154 \mathrm{~nm})$. Solid state UV-vis diffuse reflectance spectra were collected at room temperature in air using a UV-3600 spectrometer. A Shimadzu ICPS-7500 Inductively Coupled Plasma Emission Spectrometer (ICP-AES) was used to quantitatively determine the amounts of active metals. The thermal properties of catalysts in a flow of air were analyzed using a TG/DTA X70 thermogravimetric analyzer (NETZSCH) with evolved gas signals analysed by mass spectrometry. Cs-corrected STEM images were measured on a JEOL ARM200F microscope equipped with a probe-forming sphericalaberration corrector and a high-angle annular dark-field detector (HAADF), which allows for $\mathrm{Z}$ contrast imaging. Temperature programmed experiments were performed using a Micrometric ChemiSorb instrument with a TCD detector (Micrometric ChemiSorb 2750). For experiments, $0.1 \mathrm{~g}$ sample was placed in the center of quartz tube and pretreated at $200^{\circ} \mathrm{C}$ for $0.5 \mathrm{~h}$ in the flow of Ar to clean the surface. In the terms of TPR, the samples were cooled to room temperature and then heated in a flow of $\mathrm{H}_{2}$ and recorded. For $\mathrm{H}_{2}$-TPD, the samples were kept in $\mathrm{H}_{2}$ flow for $1 \mathrm{~h}$, and then purged by $\mathrm{Ar}$, followed by heating from $25-400^{\circ} \mathrm{C}$ at a rate of $10^{\circ} \mathrm{C} / \mathrm{min}$. A Micrometric ChemiSorb 2920 instrument equipped with $50 \mu \mathrm{L}$ loop was employed to perform CO chemisorption. A $\mathrm{CO} / \mathrm{Ni}$ stoichiometry of 1 was assumed for the calculation of dispersion (Table S1). X-ray Photoelectron Spectroscopy (XPS) characterization was 
conducted on the ESCALAB250 spectrometer with radiation of $\mathrm{Al} \mathrm{K \alpha}$ with the $\mathrm{C} 1 \mathrm{~s}$ peak at $284.6 \mathrm{eV}$ used as the calibration peak. Diffuse Reflectance Fourier Transform Infrared Spectroscopy of $\mathrm{CO}$ and $\mathrm{C}_{3} \mathrm{H}_{4}$ were carried out on a Bruker Tensor 27 instrument with a highly sensitive MCT detector and a diffuse IR heating chamber equipped with $\mathrm{KBr}$ windows. The sample was pressed normally into a pellet for diffuse reflectance and pretreated with $\mathrm{Ar}$ at $60^{\circ} \mathrm{C}$ for $30 \mathrm{~min}$, followed by the recording of a background at a resolution of $4 \mathrm{~cm}^{-1}$ with 64 times scanning. The sample was exposed to a $\mathrm{CO}$ or $\mathrm{C}_{3} \mathrm{H}_{4}$ (with $\mathrm{H}_{2}$ ) flow for another 30 min and purged with He before collecting the spectra. For some datasets the scan was collected after purging was used as the background spectrum in order to explore spectral changes upon heating from $30-120^{\circ} \mathrm{C}$. X-ray Absorption Spectra (XAS) at Ni K-edge were collected at the Singapore Synchrotron Light Source (SSLS) center, where a pair of channel-cut Si (111) crystals were employed in the monochromator. Ni K-edge XANES data were recorded in transmission mode with $\mathrm{Ni}$ foil and $\mathrm{NiO}$ as references. The storage ring was working at an energy of $2.5 \mathrm{GeV}$ with an average electron current of below $200 \mathrm{~mA}$. The acquired EXAFS data were processed according to the standard procedures using ATHENA and ARTEMIS modules implemented in the FEFIT software package.

Computational method. Vienna Ab Initio Package (VASP) $)^{1,2}$ was employed to perform the spin-polarized Density Functional Theory (DFT) calculations within the Generalized Gradient Approximation (GGA) in the PBE formulation ${ }^{3}$. Projected Augmented Wave (PAW) potentials ${ }^{4,5}$ were chosen to describe the ionic cores and take valence electrons into account using a plane wave basis set with a kinetic energy cutoff of $400 \mathrm{eV}$. Partial occupancies of Kohn-Sham orbitals were allowed using a Gaussian smearing method with a width of $0.05 \mathrm{eV}$. The electronic energy was considered as selfconsistent once the energy change was smaller than $10^{-7} \mathrm{eV}$. A geometry optimization was considered convergent when the energy change and force were smaller than $10^{-6}$ $\mathrm{eV}$ and $0.02 \mathrm{eV} / \AA \AA$, respectively. Grimme's DFT-D3 methodology ${ }^{6}$ was used to describe the dispersion interactions.

The equilibrium lattice constants of hexagonal $\mathrm{Mo}_{3} \mathrm{~S}_{4}$ unit cell were optimized, when using a $3 \times 3 \times 3$ Monkhorst-Pack k-point grid for Brillouin zone sampling, to be 
$\mathrm{a}=\mathrm{b}=9.167 \AA, \mathrm{c}=10.848 \AA, \alpha=\beta=90^{\circ}$, and $\gamma=120^{\circ}$. These values were then used to construct an $\mathrm{Mo}_{3} \mathrm{~S}_{4}(101)$ surface model with $\mathrm{p}(1 \mathrm{x} 2)$ periodicity in the $\mathrm{x}$ and $\mathrm{y}$ directions and one stoichiometric layer in the $\mathrm{z}$ direction by vacuum depth of $15 \AA$ in order to separate the surface slab from its periodic duplicates. This $\mathrm{Mo}_{3} \mathrm{~S}_{4}(101)$ surface model contains $24 \mathrm{Mo}$ and $32 \mathrm{~S}$ atoms. Ni atom bonded with $\mathrm{S}$ and Mo atom on the edge site. During structural optimizations, a $2 \times 1 \times 1 \mathrm{k}$-point grid in the Brillouin zone was used for k-point sampling and the top stoichiometric layer was allowed to fully relax while the bottom one was fixed. The transition state of an elementary reaction step was located by the Nudged Elastic Band (NEB) method ${ }^{7}$. In the NEB method, the path between the reactants and products was discretized into a series of five structural images. The intermediate images were relaxed until the perpendicular forces were smaller than $0.02 \mathrm{eV} / \AA$. The adsorption energy ( $\left.\mathrm{E}_{\mathrm{ads}}\right)$ of adsorbate A was defined as:

$E_{\text {ads }}=E_{A / s u r f}-E_{\text {surf }}-E_{A}(g)$

where $\mathrm{E}_{\mathrm{A} / \text { surf }}, \mathrm{E}_{\text {surf }}$ and $\mathrm{E}_{\mathrm{A}(\mathrm{g})}$ are the energy of adsorbate $\mathrm{A}$ adsorbed on the surface, the energy of clean surface, and the energy of isolated A molecule in a cubic periodic box with a side length of $20 \AA$ and a $1 \times 1 \times 1$ Monkhorst-Pack k-point grid for Brillouin zone sampling, respectively.

Catalytic Testing. Catalytic behavior was evaluated in a fixed-bed microreactor at 0.1 MPa pressure. In detail, $0.5 \mathrm{~g}$ of sample was diluted with quartz sand (Aldrich, 40-70 mesh) and placed in the reactor with the inlet gas stream of $0.15 \% \mathrm{C}_{2} \mathrm{H}_{2} / 15.0 \mathrm{C}_{2} \mathrm{H}_{4} / 3.03 \%$ $\mathrm{H}_{2}$ diluted by nitrogen to obtain a gas hourly space velocity of $9900 \mathrm{~h}^{-1}$. The reactant and product concentrations were analyzed by online gas chromatography (GC) with a flame ionization detector using a PLOT capillary column $(50 \mathrm{~m} \times 0.53 \mathrm{~mm})$. Propane was used as an internal standard. Multiple data point were collected for a given set of conditions (i.e., different temperatures) to ensure reproducibility. Acetylene conversion and ethylene selectivity were calculated as follows:

$$
\text { Acetylene conversion }=\frac{\text { Acetylene(inlet)-Acetylene(outlet) }}{\text { Acetylene(inlet) }} \quad \text { equation S1 }
$$




$$
\text { Ethylene selectivity }=\frac{C_{2} H_{4} \text { (outlet) }-C_{2} H_{4} \text { (inlet) }}{C_{2} H_{2} \text { (inlet) }-C_{2} H_{2} \text { (outlet) }}
$$

equation S2

The carbon balance of the reaction was $100 \pm 0.5 \%$. To test the catalyst stability, additional tests were performed for a continuous $16 \mathrm{~h}$ period at a constant temperature of $105^{\circ} \mathrm{C}$.

\section{Results and Discussion}

In order to judge whether $\left[\mathrm{Mo}_{3} \mathrm{~S}_{4}\left(\mathrm{H}_{2} \mathrm{O}\right)_{9}\right]^{4+}$ and $\left[\mathrm{Mo}_{3} \mathrm{NiS}_{4}\left(\mathrm{H}_{2} \mathrm{O}\right)_{10}\right]^{4+}$ clusters were synthesized successfully, the as-synthesized solutions were diluted and UV-Vis spectra were collected. Fig. S1A shows two bands at 373.9 and $620.3 \mathrm{~nm}$, which are characteristic of the $\left[\mathrm{Mo}_{3} \mathrm{~S}_{4}\left(\mathrm{H}_{2} \mathrm{O}\right)_{9}\right]^{4+}$ cluster and confirms the successful preparation of this metal cluster. However, the absorption intensity at $620.3 \mathrm{~nm}$ is relatively weak which may indicate that the $\mathrm{Mo}^{4+}$ species in the $\left[\mathrm{Mo}_{3} \mathrm{~S}_{4}\left(\mathrm{H}_{2} \mathrm{O}\right)_{9}\right]^{4+}$ cluster is partially oxidized to $\mathrm{Mo}^{6+}$. After introducing Ni into $\left[\mathrm{Mo}_{3} \mathrm{~S}_{4}\left(\mathrm{H}_{2} \mathrm{O}\right)_{9}\right]^{4+}$ cluster, a more noticeable absorbance appears at $662.0 \mathrm{~nm}$ (Fig. S1B). This is consistent with previous reports on the $\left[\mathrm{Mo}_{3} \mathrm{NiS}_{4}\left(\mathrm{H}_{2} \mathrm{O}\right)_{10}\right]^{4+}$ cluster $^{8}$.
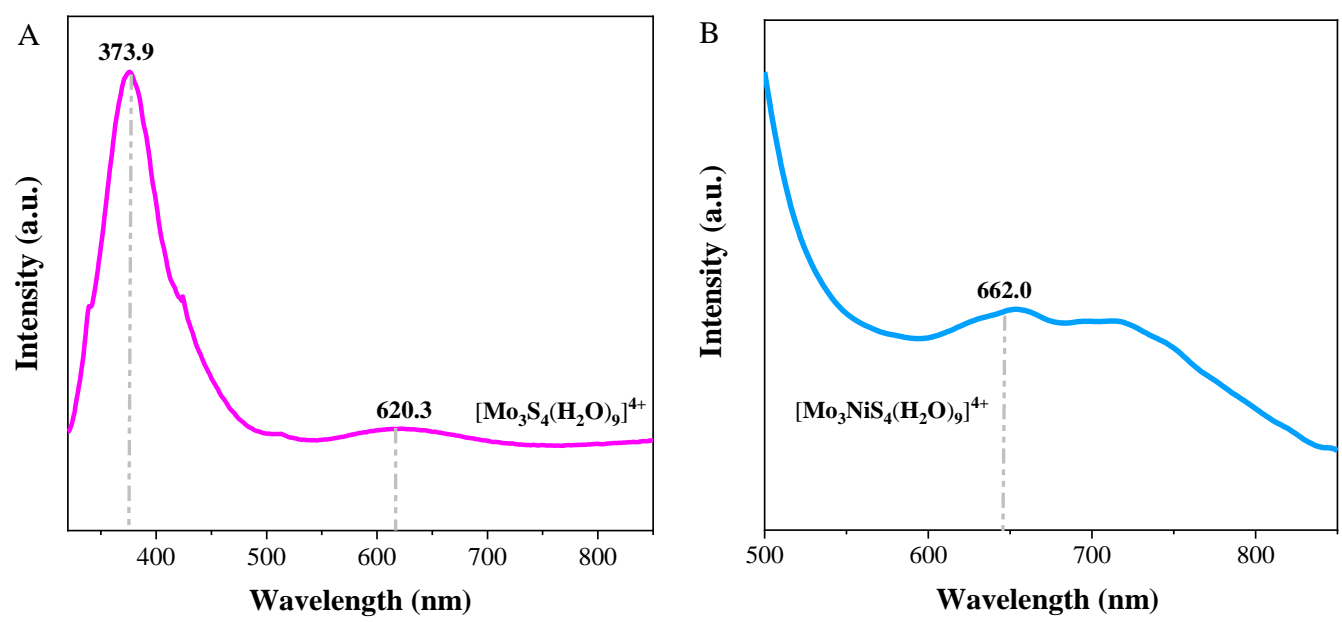

Fig.S1 UV-vis spectra of (A) $\left[\mathrm{Mo}_{3} \mathrm{~S}_{4}\left(\mathrm{H}_{2} \mathrm{O}\right)_{9}\right]^{4+}$ and (B) $\left[\mathrm{Mo}_{3} \mathrm{NiS}_{4}\left(\mathrm{H}_{2} \mathrm{O}\right)_{10}\right]^{4+}$ clusters

The $\left[\mathrm{Mo}_{3} \mathrm{NiS}_{4}\left(\mathrm{H}_{2} \mathrm{O}\right)_{10}\right]^{4+}$ cluster was subsequently supported on $\mathrm{Al}_{2} \mathrm{O}_{3}$ to obtain a $\left[\mathrm{Mo}_{3} \mathrm{NiS}_{4}\left(\mathrm{H}_{2} \mathrm{O}\right)_{10}\right]^{4+} / \mathrm{Al}_{2} \mathrm{O}_{3}$ precursor. Using TG-MS analysis it was possible to analyze the structural changes during a thermal treatment in nitrogen. As shown in Fig. S2, when the temperature rises above $c a \cdot 150^{\circ} \mathrm{C}$ an obvious weight loss occurs which corresponds to the removal of $\mathrm{H}_{2} \mathrm{O}$ (confirmed by MS). The water loss is not 
accompanied by loss of sulfur at any temperature. Consequently, $\left.\mathrm{Mo}_{3} \mathrm{NiS}_{4}\left(\mathrm{H}_{2} \mathrm{O}\right)_{10}\right]^{4+} / \mathrm{Al}_{2} \mathrm{O}_{3}$ precursor was treated at $200^{\circ} \mathrm{C}$ in $\mathrm{N}_{2}$ for $1 \mathrm{~h}$ to facilitate the removal of a large amount of water whilst retaining sulfur before a final treatment at $300{ }^{\circ} \mathrm{C}$ in $\mathrm{H}_{2}$ for $2 \mathrm{~h}$ to obtain $\mathrm{Ni}_{1} \mathrm{MoS} / \mathrm{Al}_{2} \mathrm{O}_{3}$ catalyst.

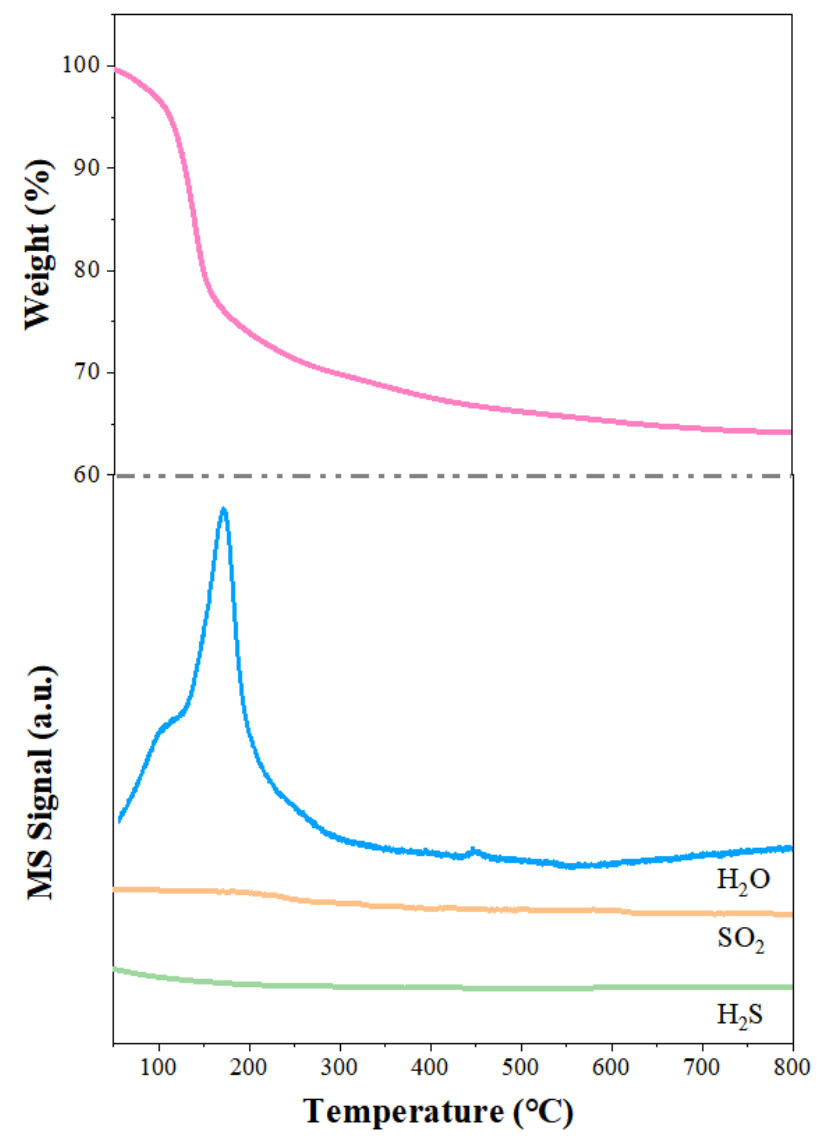

Fig. S2 TG-MS spectra of $\left[\mathrm{Mo}_{3} \mathrm{NiS}_{4}\left(\mathrm{H}_{2} \mathrm{O}\right)_{10}\right]^{4+} / \mathrm{Al}_{2} \mathrm{O}_{3}$ precursors obtained in $\mathrm{N}_{2}$ atmosphere
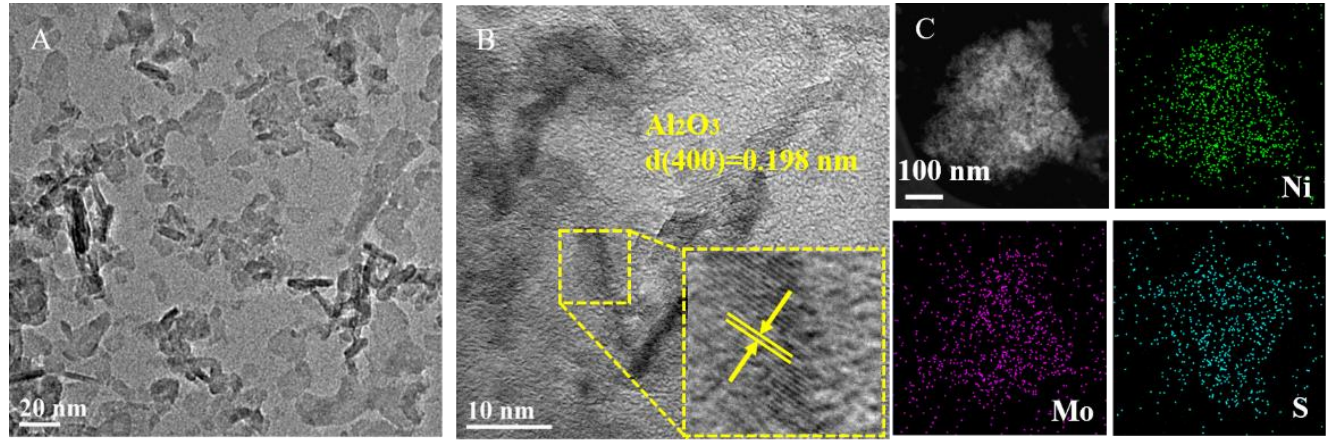

Fig. S3 (A, B) Representative HRTEM images (C) STEM-EDS elemental mapping of $\left[\mathrm{Mo}_{3} \mathrm{NiS}_{4}\left(\mathrm{H}_{2} \mathrm{O}\right)_{10}\right]^{4+} / \mathrm{Al}_{2} \mathrm{O}_{3}$ precursor. 

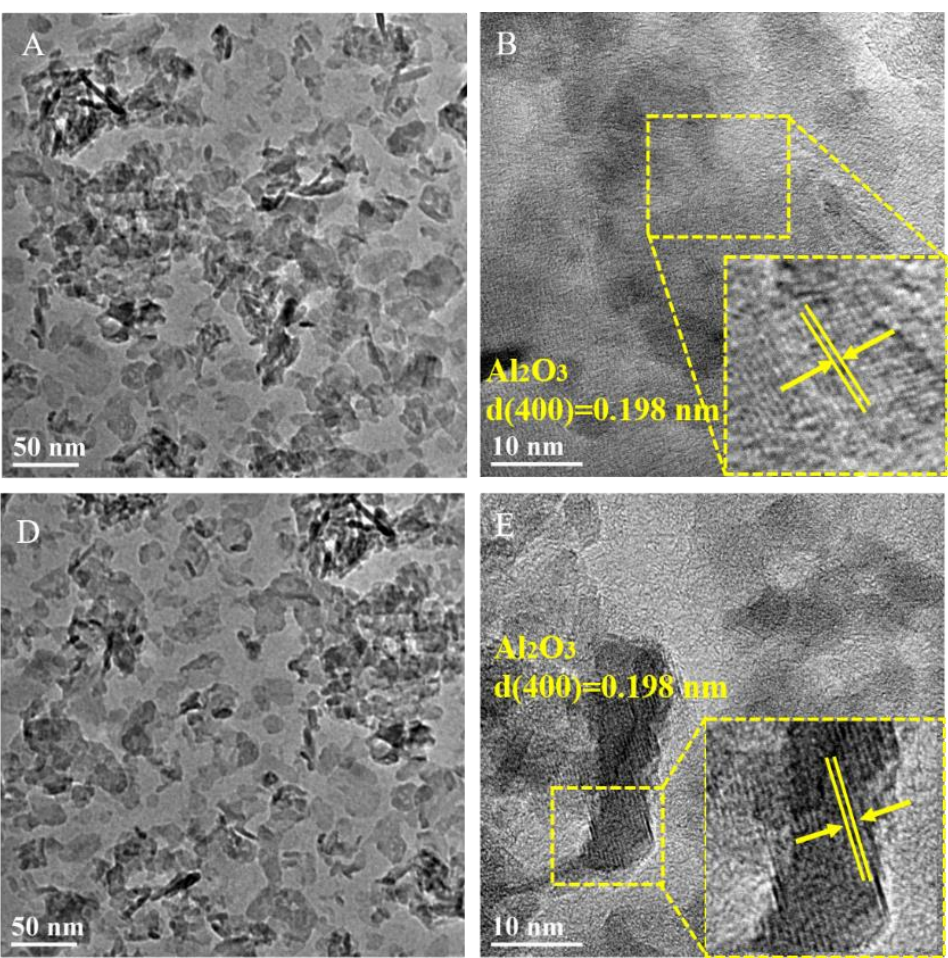
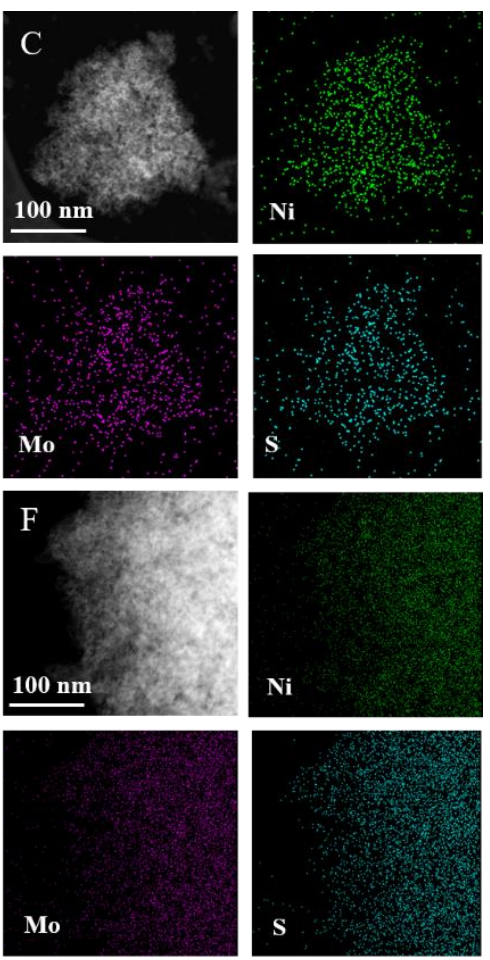

Fig. S4 (A, B, D, E) Representative HRTEM images and (C, F) STEM-EDS elemental mapping of $\mathrm{Ni}{ }_{1} \mathrm{MoS} / \mathrm{Al}_{2} \mathrm{O}_{3}$ catalyst in different regions

Fig. S5 shows the XRD patterns of the catalysts. Peaks observed at $32.5^{\circ}, 36.9^{\circ}$, $39.4^{\circ}, 45.8^{\circ}, 60.4^{\circ}$ and $67.0^{\circ}$ are the typical $\mathrm{Al}_{2} \mathrm{O}_{3}$ support used in this work. ${ }^{9}$ For $\mathrm{MoS} / \mathrm{Al}_{2} \mathrm{O}_{3}$ sample, diffraction peaks are observed at $31.5^{\circ}, 35.4^{\circ}$ and $46.7^{\circ}$ which is consistent with the presence of a $\mathrm{Mo}_{3} \mathrm{~S}_{4}$ phase (JCPDS No. 82-1709). The as-prepared $\left[\mathrm{Mo}_{3} \mathrm{NiS}_{4}\left(\mathrm{H}_{2} \mathrm{O}\right)_{10}\right]^{4+} / \mathrm{Al}_{2} \mathrm{O}_{3}$ sample (i.e., without any thermal treatment) presents an XRD pattern which is basically same as that of $\left[\mathrm{Mo}_{3} \mathrm{~S}_{4}\left(\mathrm{H}_{2} \mathrm{O}\right)_{10}\right]^{4+} \cdot{ }^{10,11}$ This indicates that the structure of cluster is essentially retained after the introduction of Ni. After thermal treatment of this sample, the characteristic peaks of $\mathrm{Al}_{2} \mathrm{O}_{3}$ support and $\mathrm{Mo}_{3} \mathrm{~S}_{4}$ still appear but without any observation from Ni species. It is therefore speculated that $\mathrm{Ni}$ enters the $\mathrm{Mo}_{3} \mathrm{~S}_{4}$ cluster and bonds Mo or $\mathrm{S}$ atoms and thus forms an isolated Ni site. In the $\mathrm{NiS} / \mathrm{Al}_{2} \mathrm{O}_{3}$ sample, it can be seen that diffraction peaks appear at $29.0^{\circ}, 32.2^{\circ}$, $37.0^{\circ}, 39.2^{\circ}$ and $45.8^{\circ}$ which are assigned to the (110), (040), (130), (112) and (132) facets in a $\mathrm{Ni}_{3} \mathrm{~S}_{2}$ phase (ICSD No. 180767). Since the loading of Mo in our $\mathrm{Mo} / \mathrm{Al}_{2} \mathrm{O}_{3}$ sample is only $0.5 \%$, any Mo phase would appear to be below what can be detected by XRD. As such, the $\mathrm{Mo} / \mathrm{Al}_{2} \mathrm{O}_{3}$ sample only shows the characteristic peaks of $\mathrm{Al}_{2} \mathrm{O}_{3}$. 

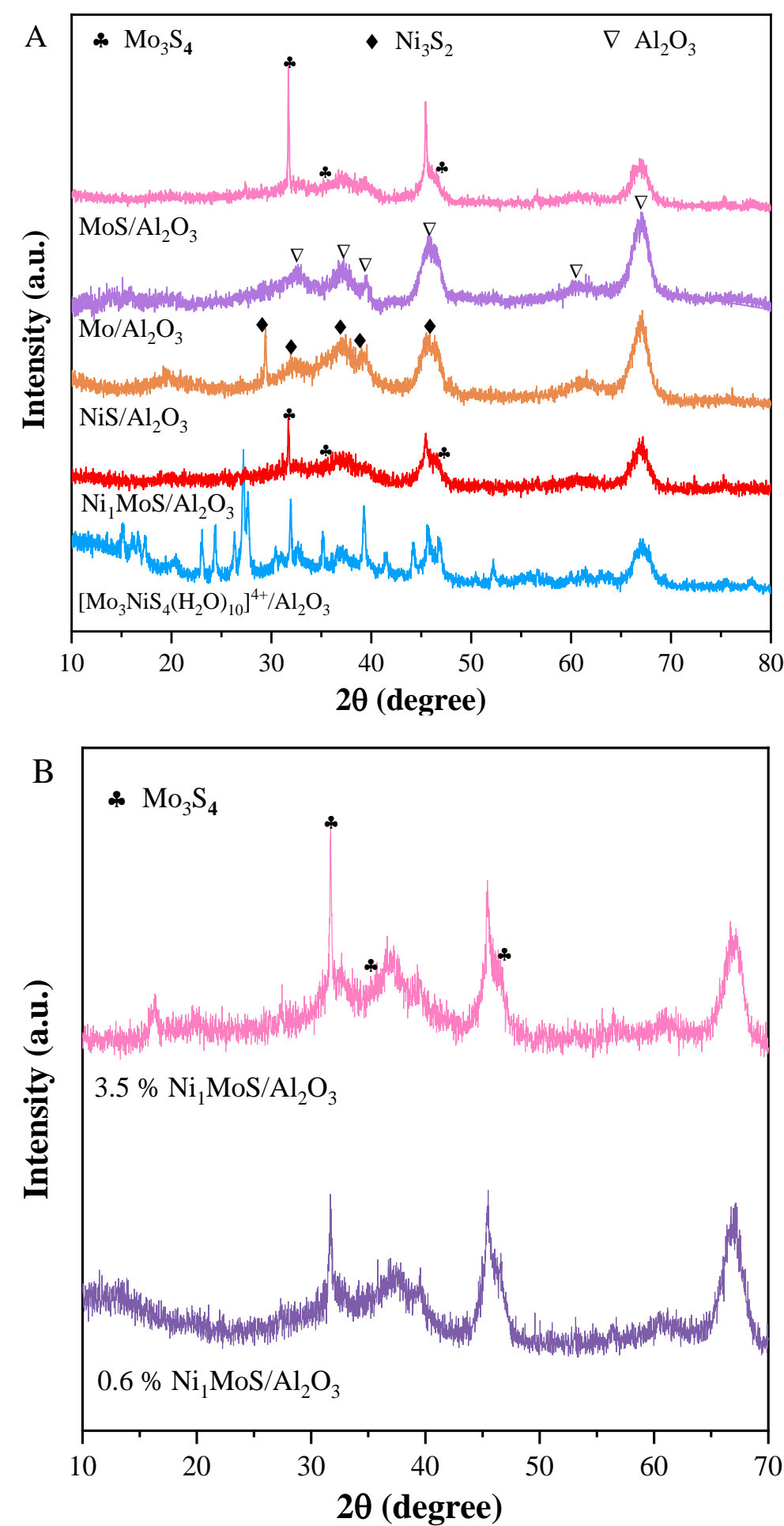

Fig. S5 XRD patterns of (A) $\mathrm{Al}_{2} \mathrm{O}_{3}$ supported Ni-based catalysts and (B) $\mathrm{Ni}_{1} \mathrm{MoS} / \mathrm{Al}_{2} \mathrm{O}_{3}$ catalyst with different Ni metal loading involving $0.6 \mathrm{wt} . \%$ and $3.5 \mathrm{wt} . \%$ 

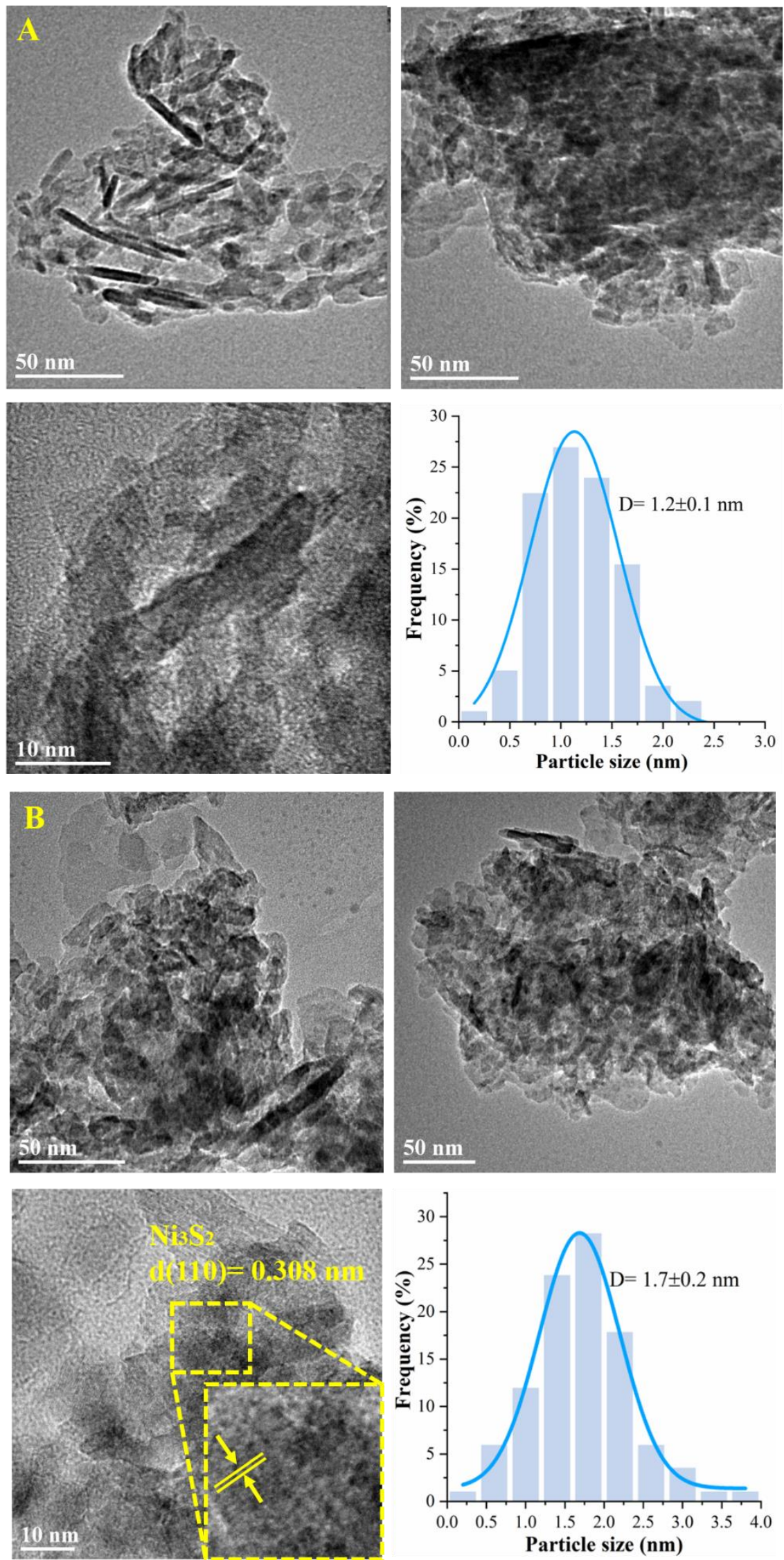

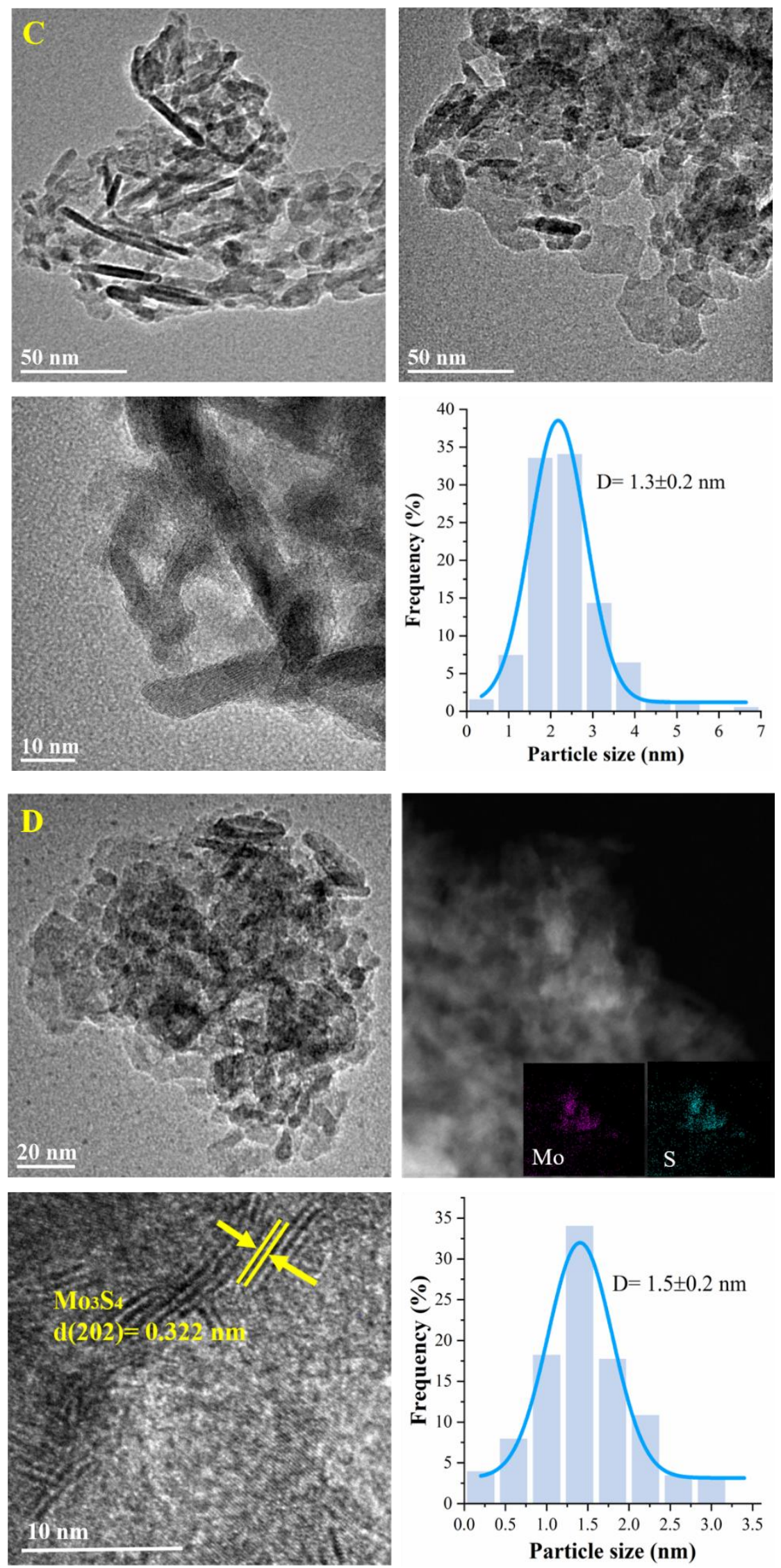

Fig. S6 Representative HRTEM images and size distribution of (A) $\mathrm{Ni} / \mathrm{Al}_{2} \mathrm{O}_{3}$, (B) $\mathrm{NiS} / \mathrm{Al}_{2} \mathrm{O}_{3}$, (C) $\mathrm{Mo} / \mathrm{Al}_{2} \mathrm{O}_{3}$ and (D) $\mathrm{MoS} / \mathrm{Al}_{2} \mathrm{O}_{3}$ catalysts 
Table S1 Physicochemical properties of the catalysts

\begin{tabular}{cccc}
\hline Catalysts & Ni loading $(\%)$ & Size $(\mathbf{n m})^{\mathbf{a}}$ & $\mathbf{D}(\%)^{\mathbf{b}}$ \\
\hline $0.5 \% \mathrm{MoNiS}_{\mathrm{Al}} \mathrm{O}_{3}$ & 0.6 & - & 100 \\
$2.5 \% \mathrm{MoNiS} / \mathrm{Al}_{2} \mathrm{O}_{3}$ & 2.5 & - & 100 \\
$3.5 \% \mathrm{MoNiS}^{\mathrm{A}} \mathrm{Al}_{2} \mathrm{O}_{3}$ & 3.5 & - & 100 \\
$\mathrm{Ni} / \mathrm{Al}_{2} \mathrm{O}_{3}$ & 2.2 & $1.2 \pm 0.1$ & 29.8 \\
$\mathrm{NiS} / \mathrm{Al}_{2} \mathrm{O}_{3}$ & 2.4 & $1.7 \pm 0.2$ & 20.1 \\
$\mathrm{Mo} / \mathrm{Al}_{2} \mathrm{O}_{3}$ & - & $1.3 \pm 0.2$ & 17.7 \\
$\mathrm{MoS} / \mathrm{Al}_{2} \mathrm{O}_{3}$ & - & $1.5 \pm 0.2$ & 27.6 \\
\hline
\end{tabular}

a Mean nanoparticle size is determined by HRTEM images.

${ }^{\mathrm{b}}$ Calculated by the particle sizes based on spherical geometry.

Ni dispersion (D) was calculated from

$$
d=\frac{6 A}{\rho \sigma L D}
$$

equation $\mathrm{S} 3$

where $\mathrm{d}$ is the average palladium particle size $(\mathrm{nm})$, A is atomic mass, $\rho$ is the density of $\mathrm{Ni}$ atom, $\mathrm{L}$ is the Avogadro constant, and $\sigma$ is average surface area occupied by one $\mathrm{Ni}$ atom.
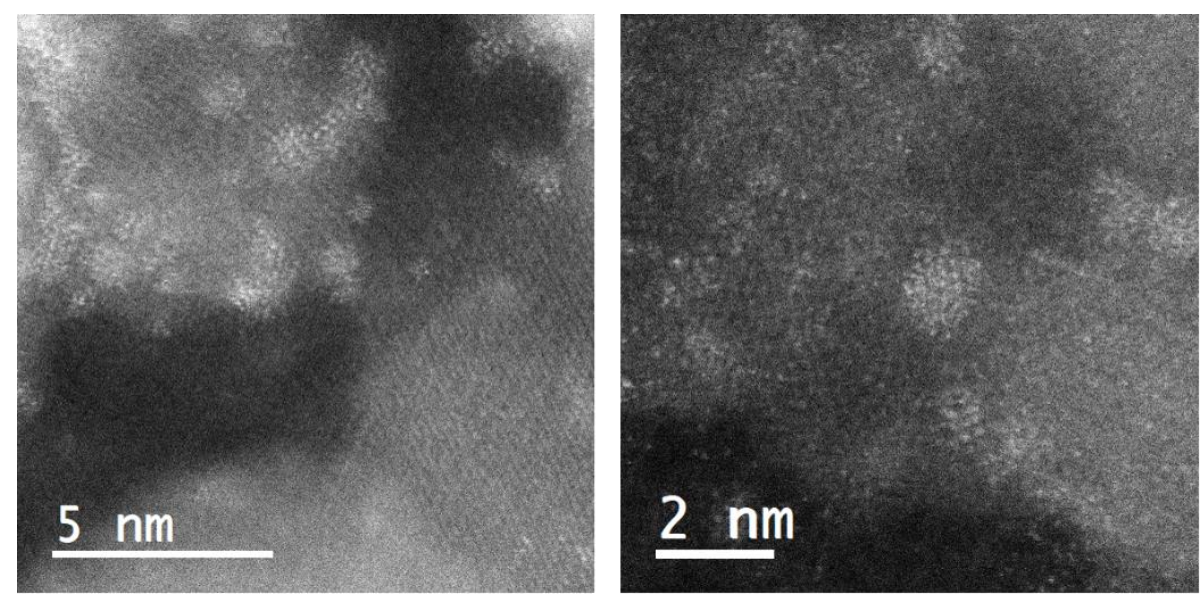

Fig. S7 AC-HAADF-STEM images of $\mathrm{Ni}_{1} \mathrm{MoS} / \mathrm{Al}_{2} \mathrm{O}_{3}$ catalyst in different regions 

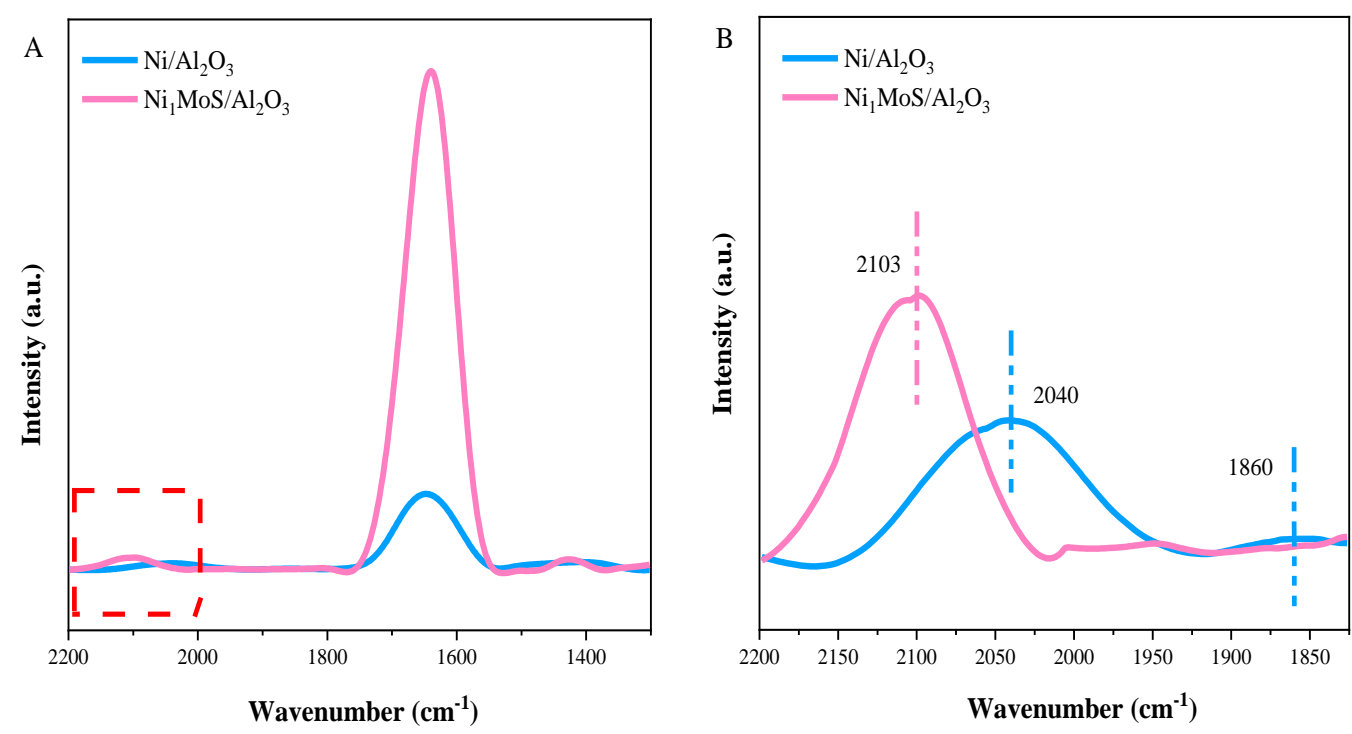

Fig. S8 (A) CO infrared spectra (B) Enlarged region in the dotted area in figure A of $\mathrm{Ni} 1 \mathrm{MoS} / \mathrm{Al}_{2} \mathrm{O}_{3}$ and $\mathrm{Ni}$ nanoparticles on $\mathrm{Al}_{2} \mathrm{O}_{3}$

X-ray Photoelectron Spectroscopy (XPS) was performed in order to investigate the electronic structures of $\mathrm{Ni}$ species. Ni $2 \mathrm{p}$ spectra of the catalysts possess two spin splitting peaks involving Ni 2 $\mathrm{p}_{3 / 2}$ and $\mathrm{Ni} 2 \mathrm{p}_{1 / 2}$ signals, which can be deconvoluted by using a Gaussian fitting method. As shown in Fig. S9, a characteristic peak is observed at ca. $856.5 \mathrm{eV}$ in Ni/Al $\mathrm{O}_{3}$ and $\mathrm{NiS} / \mathrm{Al}_{2} \mathrm{O}_{3}$ catalysts, attributed to the presence of an oxidized Ni species. Two important points could be extracted from this. Firstly, there are no characteristic peaks at $852.7 \mathrm{eV}$, which suggests that a metallic $\mathrm{Ni}$ species is absent in $\mathrm{Ni} / \mathrm{Al}_{2} \mathrm{O}_{3}$ (likely due to re-oxidation in the air storage). Secondly, the binding energy of oxidized $\mathrm{Ni}$ species in $\mathrm{NiS} / \mathrm{Al}_{2} \mathrm{O}_{3}$ catalysts is similar to that reported for $\mathrm{Ni}^{2+}$ in $\mathrm{NiO}$. This indicates that there is no obvious electron transfer between $\mathrm{Ni}$ and $\mathrm{S}$. However, the bands of oxidized $\mathrm{Ni}$ species in $\mathrm{Ni}_{1} \mathrm{MoS} / \mathrm{Al}_{2} \mathrm{O}_{3}$ occurs at $\sim 0.7 \mathrm{eV}$ lower than that of $\mathrm{NiS} / \mathrm{Al}_{2} \mathrm{O}_{3}$. This in turn suggests that the electronic configurations of $\mathrm{Ni}$ sites within the $\mathrm{Mo}_{3} \mathrm{~S}_{4}$ cluster are redistributed to form $\mathrm{Ni}^{\delta+}$ species $(\delta=0-2)$. 


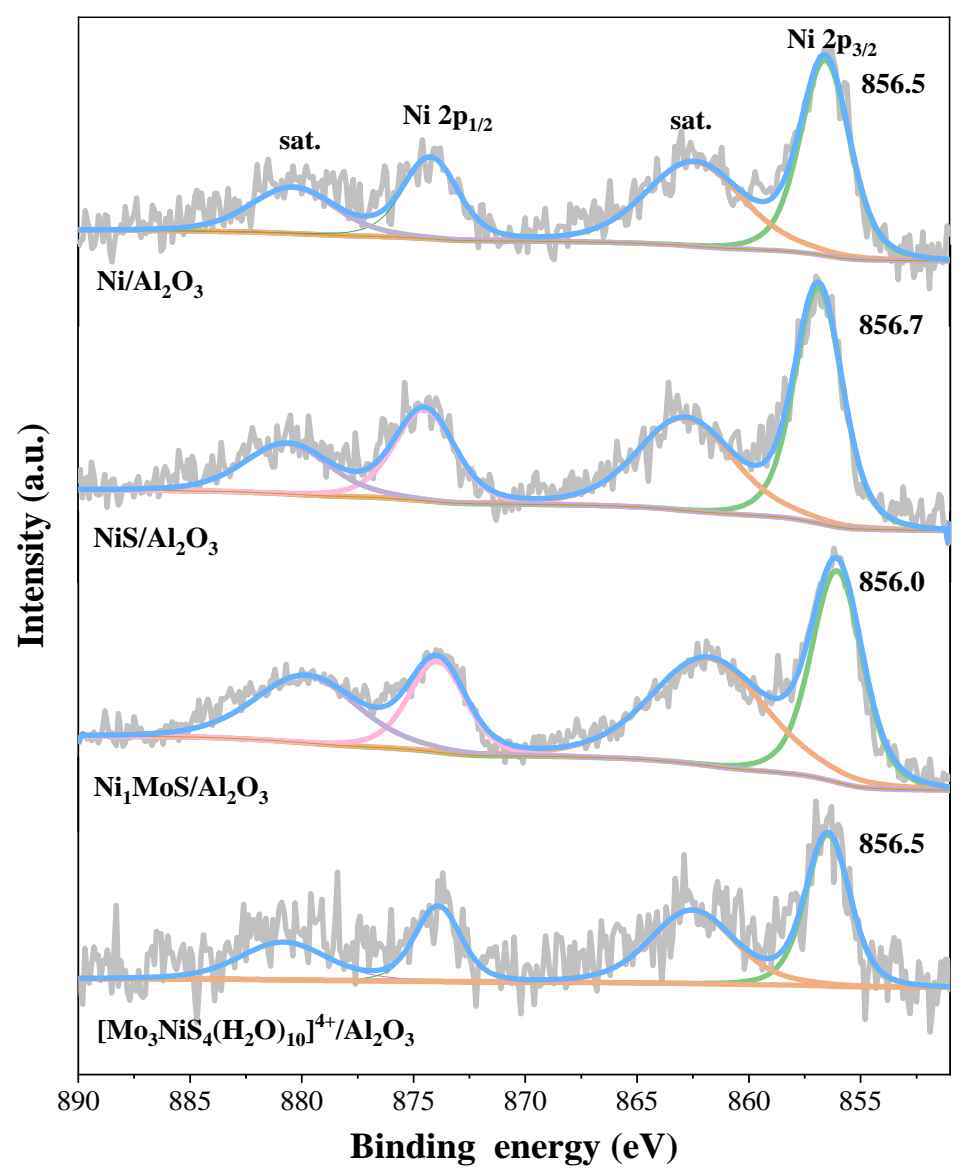

Fig. S9 Ni 2p XP spectra of Ni-based catalysts

Table S2 EXAFS parameters fitted for the samples

\begin{tabular}{ccccccc}
\hline Samples & $\begin{array}{c}\text { Scattering } \\
\text { pair }\end{array}$ & $\mathrm{CN}^{\mathrm{a}}$ & $\mathrm{r}^{\mathrm{b}}(\AA)$ & $\begin{array}{c}\Delta \mathrm{E}^{\mathrm{c}} \\
(\mathrm{eV})\end{array}$ & $\sigma^{2 \mathrm{~d}}$ & $\mathrm{R}^{\mathrm{e}}$ \\
\hline \multirow{2}{*}{$\mathrm{Ni}_{1} \mathrm{MoS} / \mathrm{Al}_{2} \mathrm{O}_{3}$} & $\mathrm{Ni}-\mathrm{S}$ & 2.7 & 2.17 & -4.7 & 0.0061 & \\
& $\mathrm{Ni}-\mathrm{Mo}$ & 1.7 & 3.20 & -4.5 & 0.0066 & 0.009 \\
& $\mathrm{Ni}-\mathrm{S}$ & 3.9 & 2.18 & -5.2 & 0.0063 & \\
$\mathrm{NiS} / \mathrm{Al}_{2} \mathrm{O}_{3}$ & $\mathrm{Ni}-\mathrm{Ni}$ & 8.8 & 2.51 & -5.3 & 0.0067 & 0.006 \\
&
\end{tabular}

${ }^{\text {a }}$ Coordination number $(\mathrm{CN})$

${ }^{\mathrm{b}}$ Atomic distance $(\mathrm{r})$

${ }^{\mathrm{c}}$ Energy difference in the absorption threshold between the reference and target samples $(\Delta \mathrm{E})$

${ }^{\mathrm{d}}$ Debye-Waller factor $\left(\sigma^{2}\right) \quad{ }^{\mathrm{e}}$ Factor value reflecting the fitting quality for XAFS analysis 

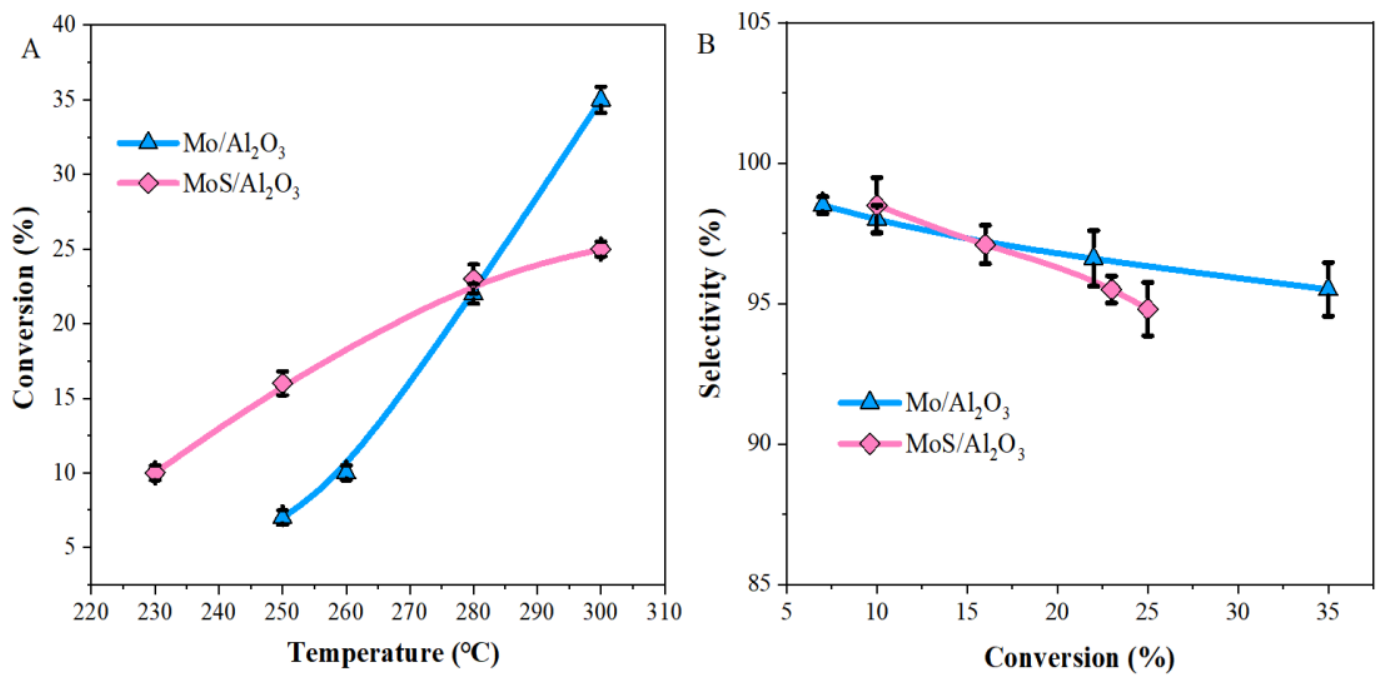

Fig. S10 (A) Acetylene conversion as a function of reaction temperature and (B) ethylene selectivity as a function of acetylene conversion over Mo-based catalysts. Error bars represent standard deviation from three independent measurements (Reaction conditions: $0.5 \mathrm{~g}$ of catalyst, $20: 1$ ratio of $\mathrm{H}_{2} / \mathrm{C}_{2} \mathrm{H}_{2}$, total flow $\left.=165 \mathrm{~mL} \mathrm{~min}^{-1}, \mathrm{SV}=9900 \mathrm{~h}^{-1}\right)$.

Table S3 Comparison of catalytic performance over non-noble metal-based catalysts

\begin{tabular}{|c|c|c|c|c|c|c|c|}
\hline Catalysts & $\mathrm{T}\left({ }^{\circ} \mathrm{C}\right)$ & $\begin{array}{c}\mathrm{H}_{2} / \\
\mathrm{C}_{2} \mathrm{H}_{2}\end{array}$ & $\begin{array}{l}\text { SV } \\
\left(\mathrm{h}^{-1}\right)\end{array}$ & $\begin{array}{l}\text { Conv. } \\
\text { (\%) }\end{array}$ & $\begin{array}{l}\text { Sel. } \\
(\%)\end{array}$ & $\begin{array}{c}\text { Rate } \\
(\mathrm{mL} \\
\left.\min ^{-1} \mathrm{~g}_{\mathrm{Ni}}^{-1}\right)\end{array}$ & $\begin{array}{c}\mathrm{Ea} \\
(\mathrm{kJ} / \mathrm{mol})\end{array}$ \\
\hline $\mathrm{Ni}_{1} \mathrm{MoS} / \mathrm{Al}_{2} \mathrm{O}_{3}$ & 125 & $20: 1$ & 9900 & 100 & 90 & $2000^{\mathrm{a}}$ & 28.3 \\
\hline $\mathrm{NiCu} / \mathrm{SiO}_{2}{ }^{12}$ & 140 & $4: 1$ & 30000 & 100 & 10 & 2500 & -- \\
\hline $\mathrm{Ni} / \mathrm{MCM}-41^{13}$ & 250 & $2: 1$ & 8000 & 100 & 47 & 1667 & -- \\
\hline $\mathrm{Ni}_{0.25} / \mathrm{SiO}_{2}{ }^{14}$ & 200 & $20: 1$ & 60000 & 100 & -200 & 1586 & -- \\
\hline $\mathrm{AgNi}_{0.125} / \mathrm{SiO}_{2}{ }^{14}$ & 200 & $20: 1$ & 60000 & 100 & 31.4 & 973 & -- \\
\hline Ni SAs/N-C 15 & 200 & $10: 1$ & 6000 & 96 & 90 & -- & -- \\
\hline $\mathrm{MoP}^{16}$ & 240 & $10: 1$ & 36000 & 99 & 73 & -- & -- \\
\hline $\mathrm{NiZn} / \mathrm{SiO}_{2}{ }^{17}$ & 200 & $10: 1$ & 128000 & 15 & 80 & 1000 & 27.5 \\
\hline $\mathrm{NiGa}^{18}$ & 190 & $10: 1$ & 144000 & 90 & 82 & 216 & -- \\
\hline $\mathrm{AgNi}_{0.5} / \mathrm{SiO}_{2}{ }^{19}$ & 180 & $20: 1$ & 60000 & 100 & 55 & 917 & 52.8 \\
\hline $\mathrm{CuNi}_{0.125} / \mathrm{SiO}_{2}{ }^{19}$ & 180 & $20: 1$ & 60000 & 100 & 50 & 2000 & 54.5 \\
\hline $\mathrm{Ni}_{6} \mathrm{In} / \mathrm{SiO}_{2}{ }^{20}$ & 180 & $5: 1$ & 36000 & 100 & 65 & 150 & -- \\
\hline
\end{tabular}




\begin{tabular}{cccccccc}
\hline $\mathrm{Ni} / \mathrm{CeO}_{2}{ }^{21}$ & 110 & $4: 1$ & 21000 & 100 & 65 & 117 & -- \\
$\mathrm{CeNi}_{3.75} \mathrm{Ga}_{1.25}{ }^{22}$ & 90 & $139: 1$ & -- & 100 & 40 & -- & -- \\
$\mathrm{NiCu} / \mathrm{MMO}^{23}$ & 160 & $2: 1$ & 8040 & 100 & 70 & -- & 34.2 \\
$\mathrm{Au} / \mathrm{CNA}^{24}$ & 300 & $5: 1$ & 17200 & 25 & 90 & -- & -- \\
$\mathrm{Au} / \mathrm{Al}_{2} \mathrm{O}_{3}{ }^{24}$ & 300 & $5: 1$ & 17200 & 8 & 70 & -- & -- \\
\hline
\end{tabular}

${ }^{\mathrm{a}}$ In order to compare with literature, reaction rate of $\mathrm{Ni}_{1} \mathrm{MoS} / \mathrm{Al}_{2} \mathrm{O}_{3}$ catalyst in acetylene semihydrogenation is expressed as the volume of acetylene converted per min per gram Ni.

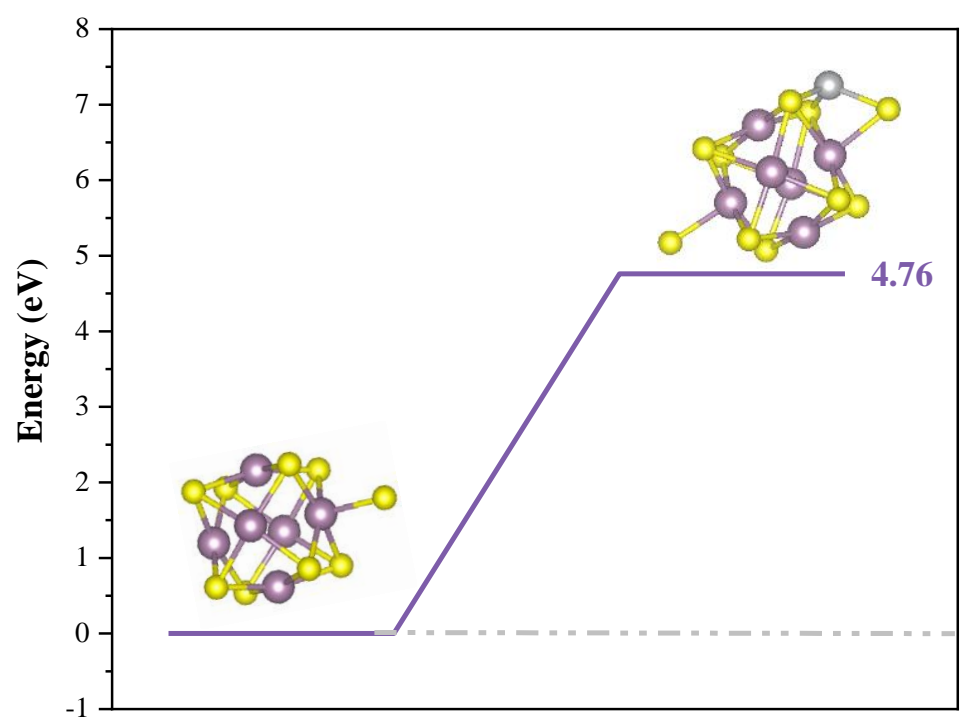

Fig. S11 Stability of Ni single atom anchored in $\mathrm{Mo}_{3} \mathrm{~S}_{4}$. The insets show the structures of the corresponding model.

TG-MS analysis was performed over fresh and spent $\mathrm{MoNiS} / \mathrm{Al}_{2} \mathrm{O}_{3}$ catalysts in air. As shown in Fig. S12, the weight loss for fresh $\mathrm{Ni}_{1} \mathrm{MoS} / \mathrm{Al}_{2} \mathrm{O}_{3}$ was about $5 \%$, which is thought to originate from the carbonate loss from the surface of the alumina support. There is no significant difference in the absolute weight loss of the fresh and spent samples (used for $16 \mathrm{~h}$ ). This suggests that only a small or negligible amount of carbonaceous species form during reaction, which demonstrates that the $\mathrm{Ni}{ }_{1} \mathrm{MoS} / \mathrm{Al}_{2} \mathrm{O}_{3}$ catalyst reported here provides excellent resistance towards carbon deposition. This in turn would imply that the catalytically active Ni species displays excellent stability as 
compared with traditionally supported $\mathrm{Ni}$ catalysts which deactivate due to carbon deposition during acetylene hydrogenation.

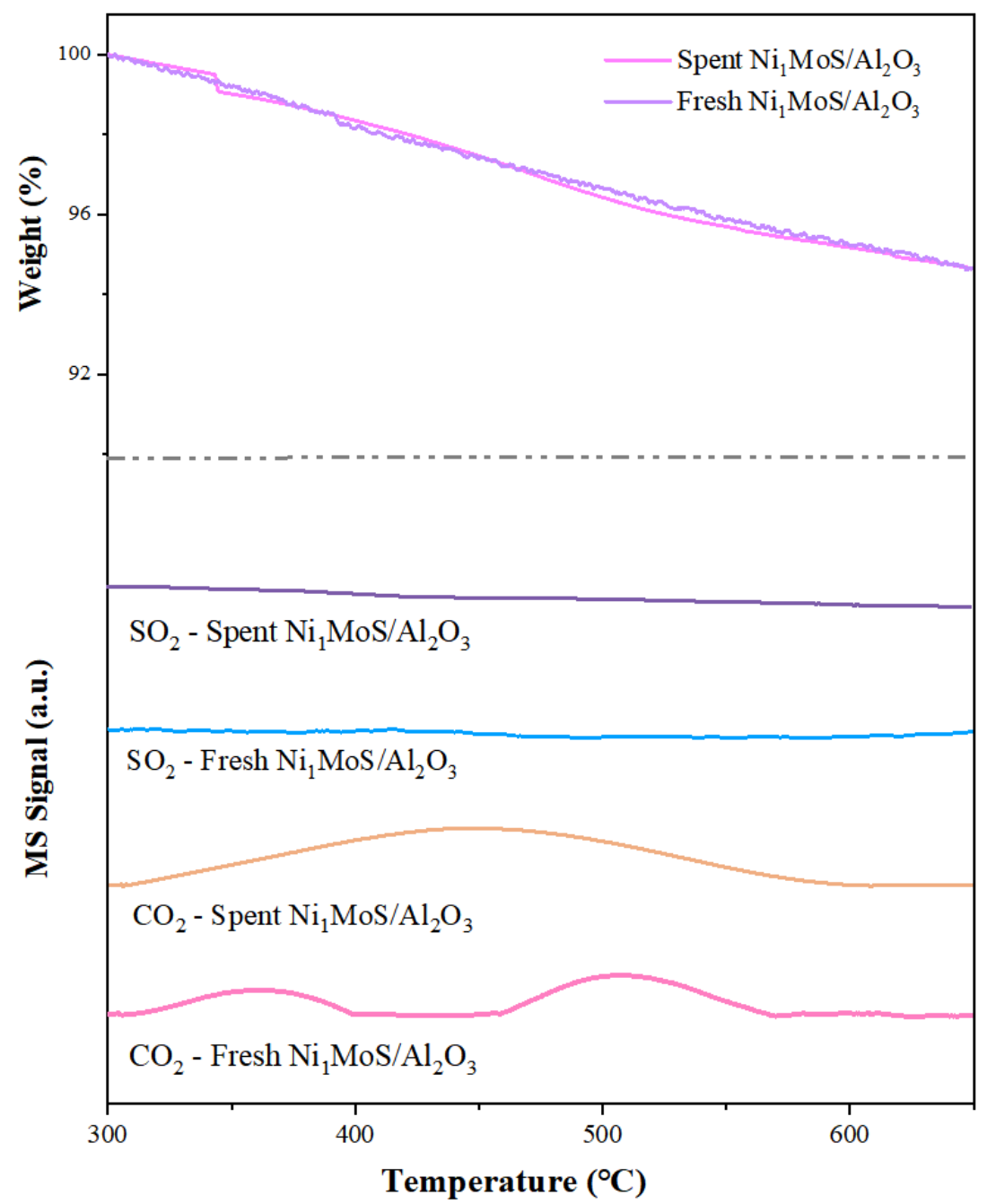

Fig. S12 TG-MS spectra obtained in air atmosphere of $\mathrm{Ni}_{1} \mathrm{MoS} / \mathrm{Al}_{2} \mathrm{O}_{3}$ before and after reaction
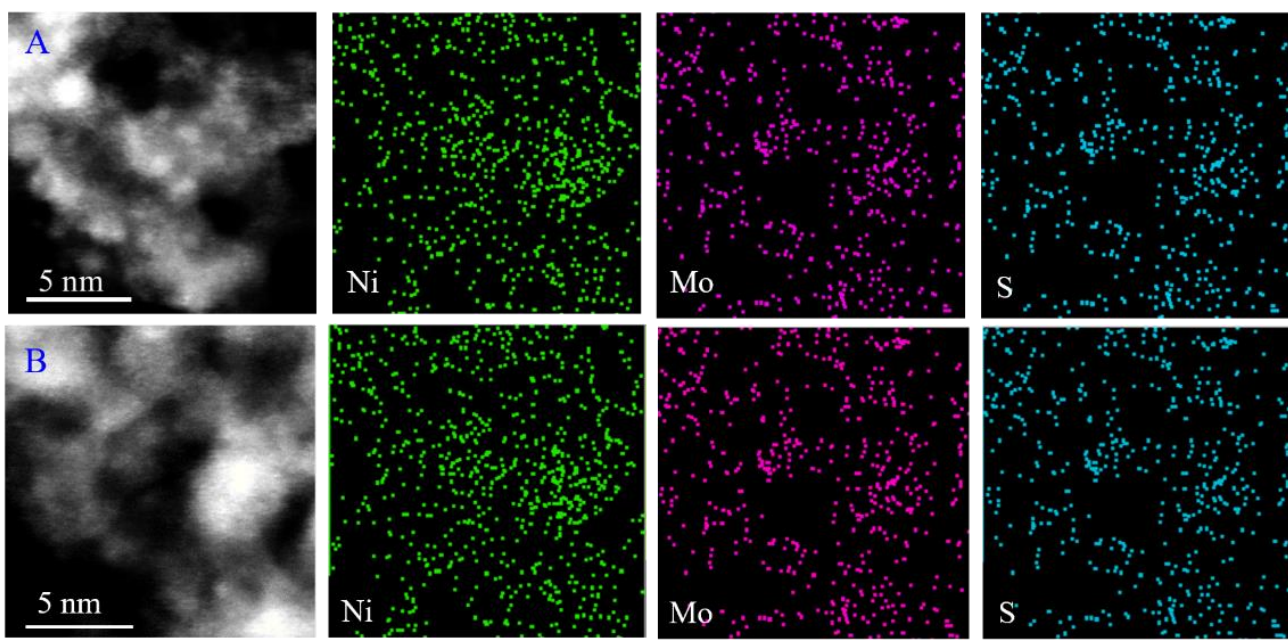

Fig. S13 (A, B) Aberration-corrected STEM images and mapping of spent $\mathrm{Ni}_{1} \mathrm{MoS} / \mathrm{Al}_{2} \mathrm{O}_{3}$ catalyst in different regions 


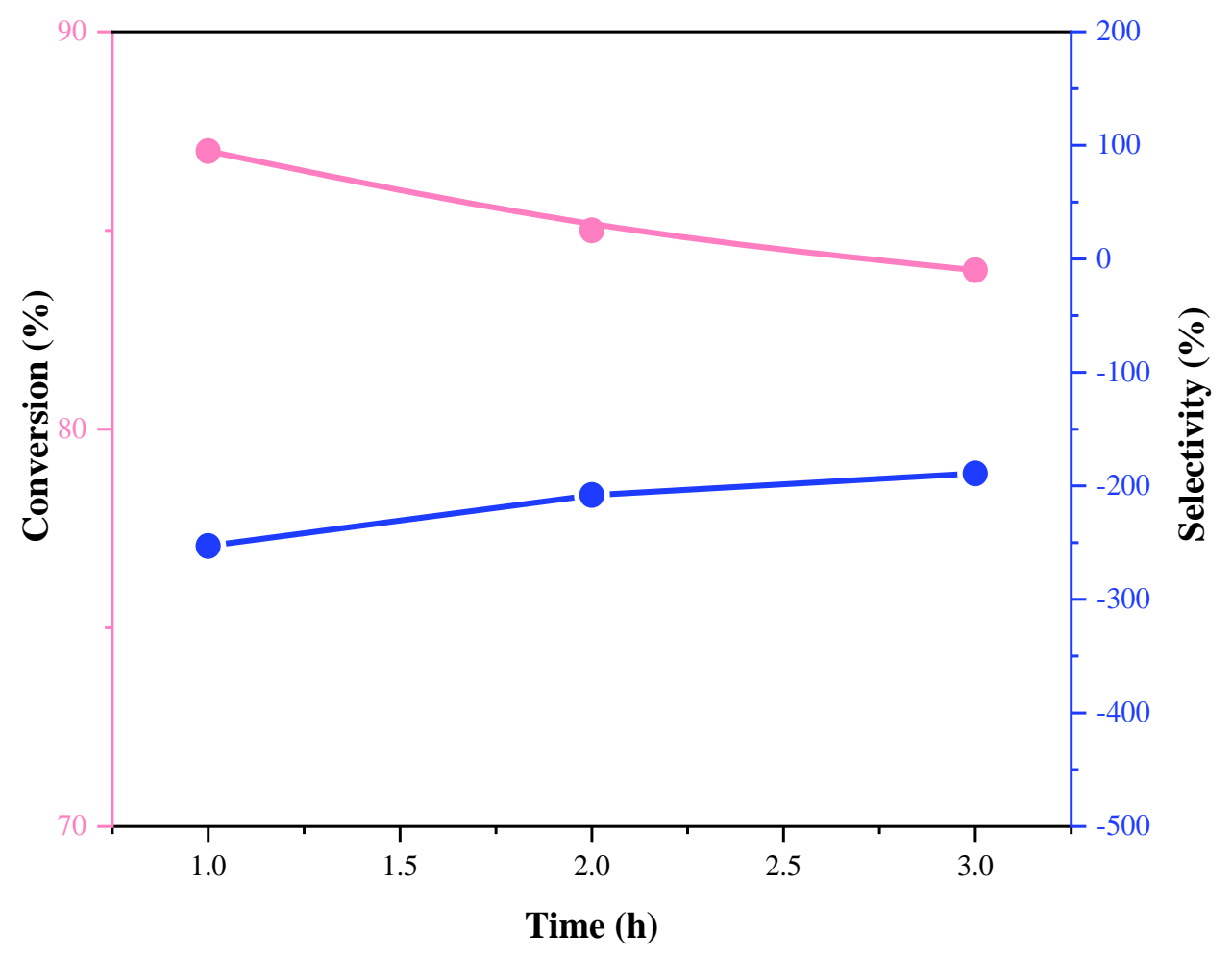

Fig. S14 Time on stream of monometallic Ni nanoparticles on $\mathrm{Al}_{2} \mathrm{O}_{3}$ for $3 \mathrm{~h}$

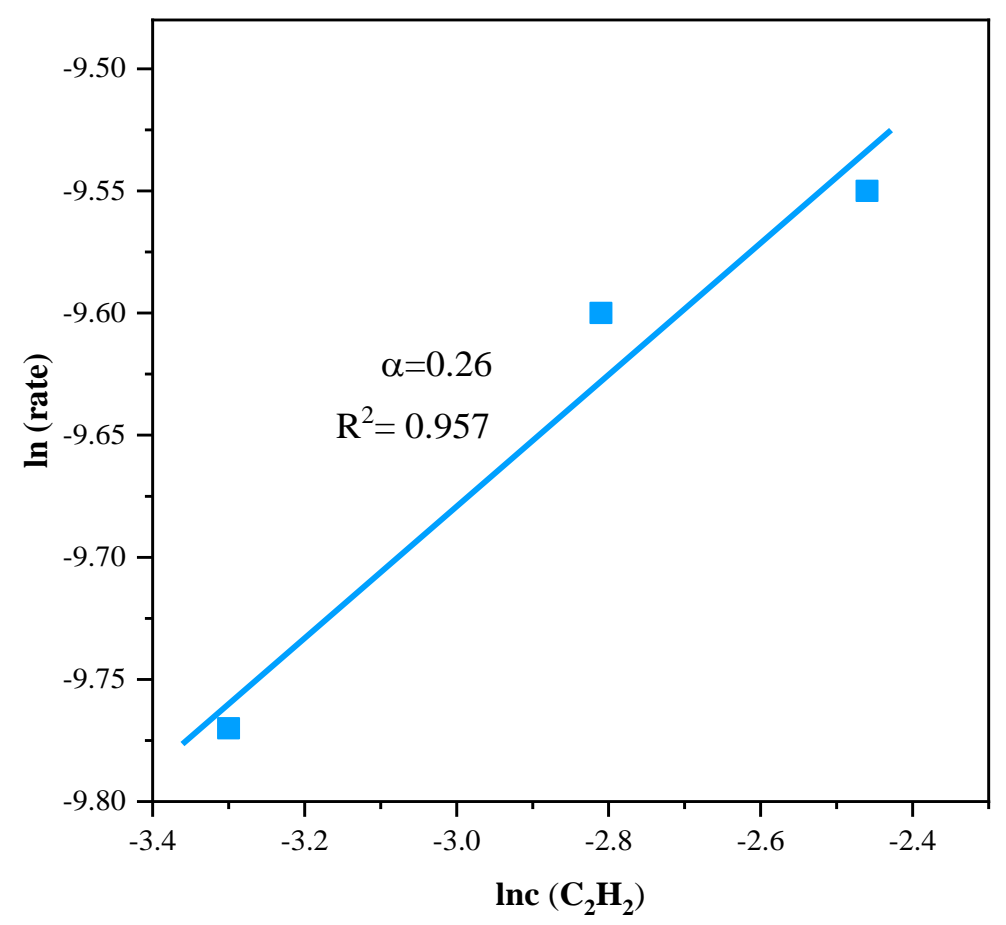

Fig. S15 Logarithmic dependence of reaction rate on the initial concentration of acetylene for determining the reaction order on Ni-based catalyst 

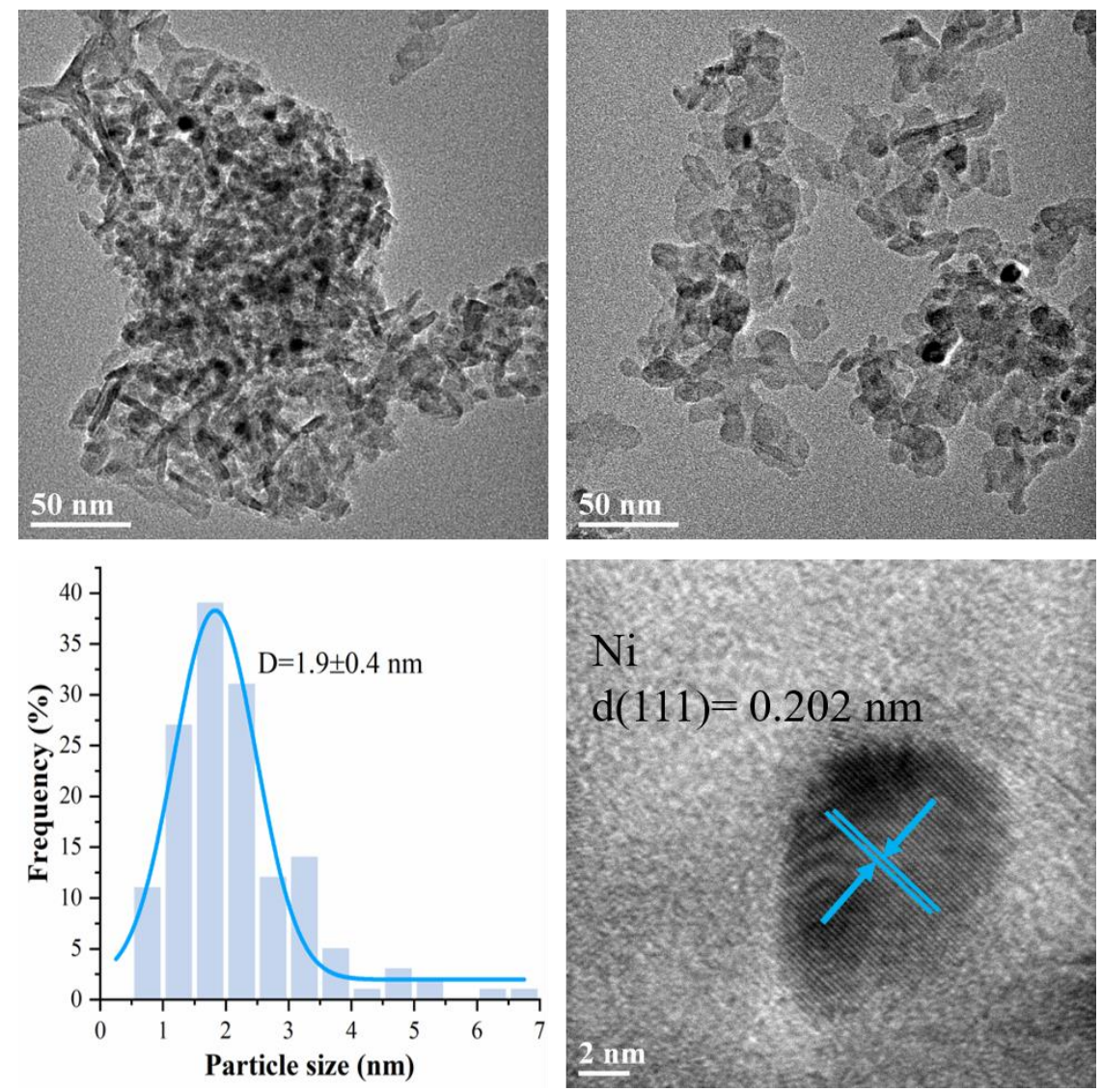

Fig. S16 STEM images and size distribution of spent $\mathrm{Ni}$ nanoparticles on $\mathrm{Ni} / \mathrm{Al}_{2} \mathrm{O}_{3}$ catalyst
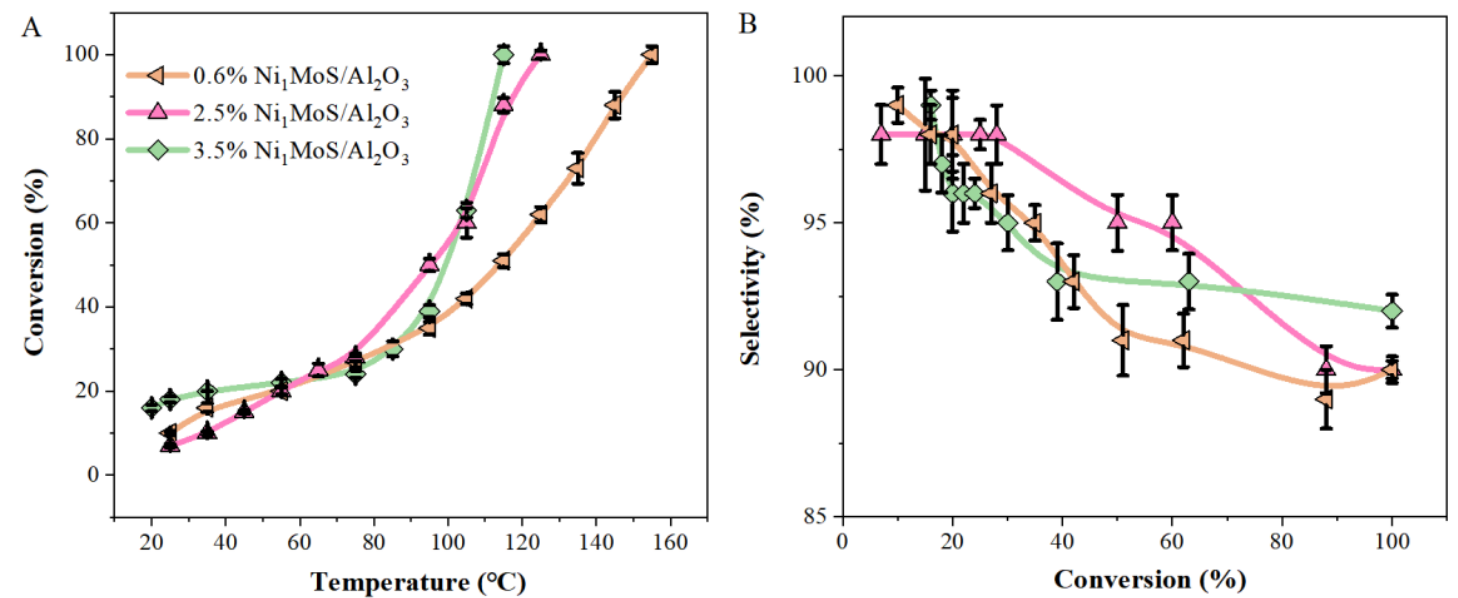

Fig. S17 The plots of (A) acetylene conversion versus temperature and (B) ethylene selectivity versus acetylene conversion over $\mathrm{Ni}_{1} \mathrm{MoS} / \mathrm{Al}_{2} \mathrm{O}_{3}$ catalyst with different $\mathrm{Ni}$ metal loading involving 0.6 wt. $\%, 2.5$ wt. $\%$ and 3.5 wt. $\%$ 

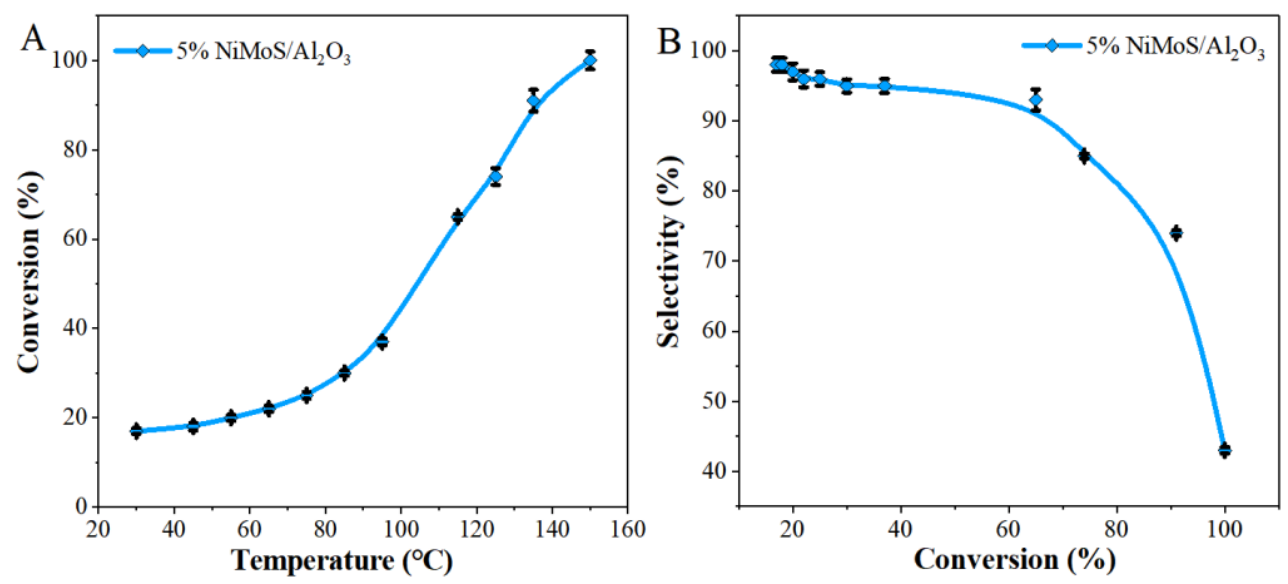

Fig. S18 (A) Acetylene conversion as a function of reaction temperature and (B) ethylene selectivity as a function of acetylene conversion over $5 \% \mathrm{NiMoS} / \mathrm{Al}_{2} \mathrm{O}_{3}$. Error bars represent standard deviation from three independent measurements (Reaction conditions: $0.5 \mathrm{~g}$ of catalyst, 20:1 ratio of $\mathrm{H}_{2} / \mathrm{C}_{2} \mathrm{H}_{2}$, total flow $=165 \mathrm{~mL} \mathrm{~min}^{-1}, \mathrm{SV}=9900 \mathrm{~h}^{-1}$ ).
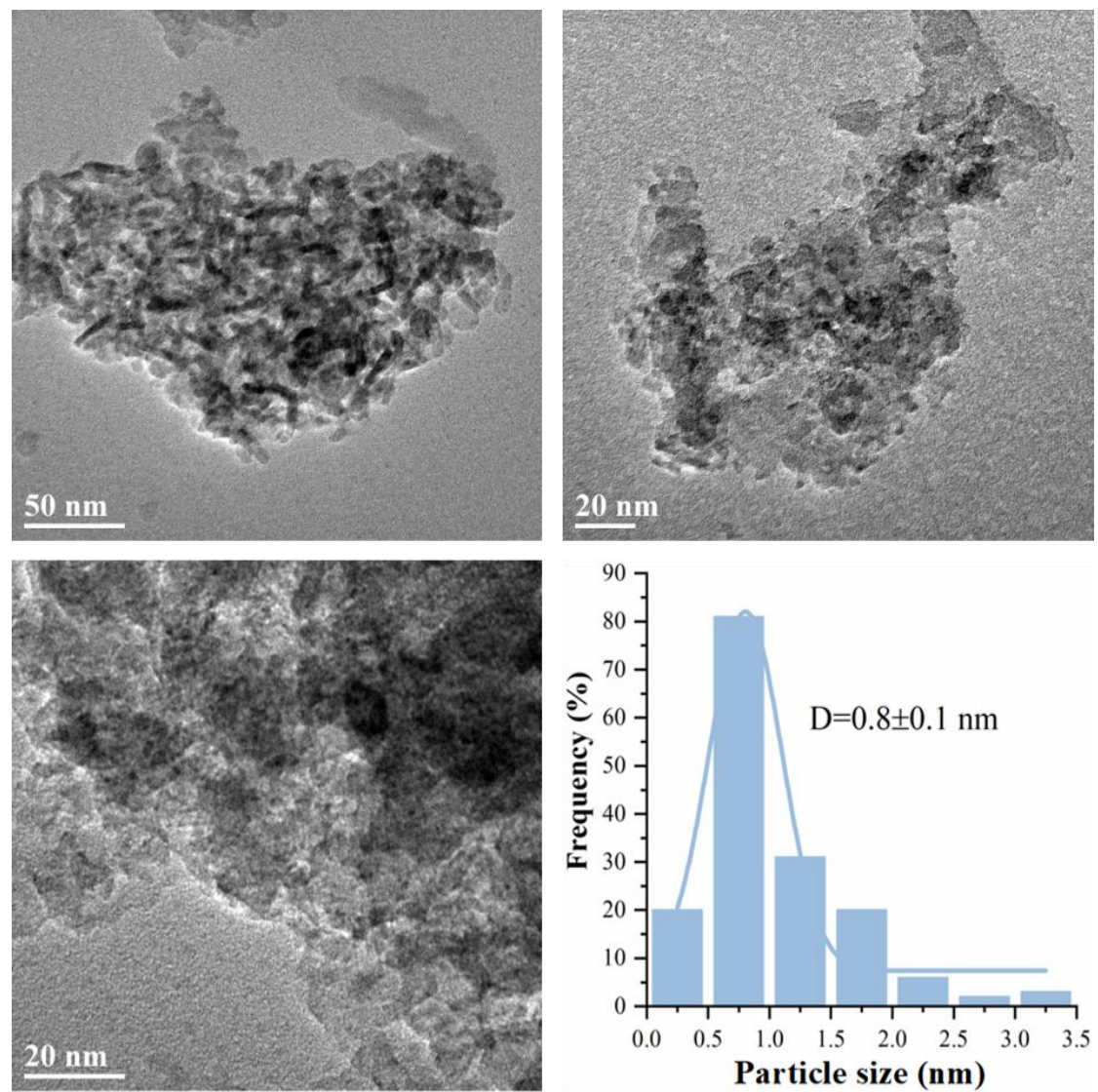

Fig. S19 Representative HRTEM images and size distribution of $5 \% \mathrm{NiMoS} / \mathrm{Al}_{2} \mathrm{O}_{3}$ 


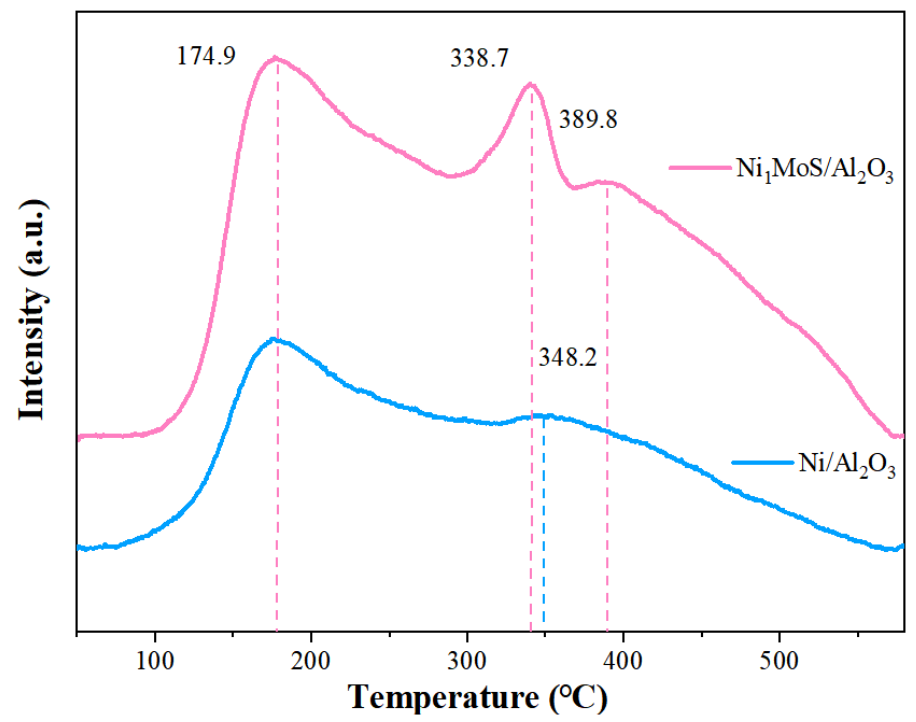

Fig. $\mathbf{S 2 0} \mathrm{H}_{2}-\mathrm{TPD}$ profiles of $\mathrm{Ni}_{1} \mathrm{MoS} / \mathrm{Al}_{2} \mathrm{O}_{3}$ and $\mathrm{Ni} / \mathrm{Al}_{2} \mathrm{O}_{3}$ catalysts

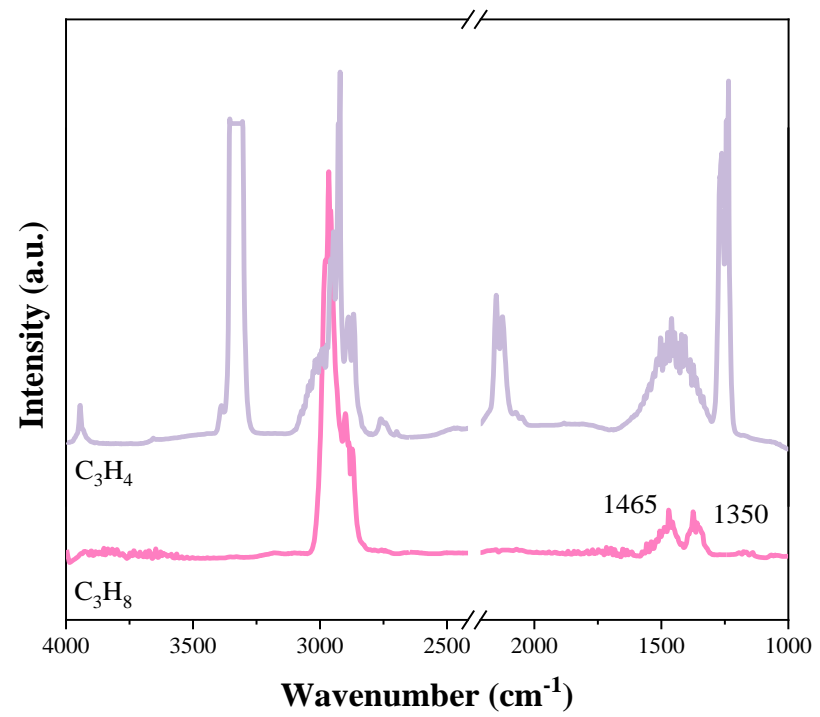

Fig. S21 Infrared spectra of propyne and propane

Moreover, the structure files in CIF format has been also provided as below:

data_VESTA_phase_1

_chemical_name_common

$\mathrm{Ni}_{1} \mathrm{MoS}$

_cell_length_a

6.410100

_cell_length_b

12.820300

_cell_length_c

26.121099

_cell_angle_alpha

90.000000

_cell_angle_beta

90.000000

_cell_angle_gamma

91.297401

_cell_volume

2146.066112

_space_group_name_H-M_alt

'P 1' 


\begin{tabular}{|c|c|c|c|c|c|}
\hline \\
\hline & & & & & \\
\hline \multicolumn{6}{|c|}{$\begin{array}{l}\text { _space_group_IT_number } \\
\text { loop_ } \\
\text { _space_group_symop_operation_xyz }\end{array}$} \\
\hline \multirow{2}{*}{\multicolumn{6}{|c|}{ 'x, y, z' }} \\
\hline \multirow{2}{*}{\multicolumn{6}{|c|}{ loop_ }} \\
\hline & & & & & \\
\hline \multicolumn{6}{|c|}{ _atom_site_label } \\
\hline \multicolumn{6}{|c|}{ _atom_site_occupancy } \\
\hline \multicolumn{6}{|c|}{ _atom_site_fract_x } \\
\hline \multicolumn{6}{|c|}{ _atom_site_fract_y } \\
\hline \multicolumn{6}{|c|}{ _atom_site_fract_z } \\
\hline \multicolumn{6}{|c|}{ _atom_site_adp_type } \\
\hline \multicolumn{6}{|c|}{ _atom_site_B_iso_or_equiv } \\
\hline \multicolumn{6}{|c|}{ _atom_site_type_symbol } \\
\hline Ni1 & 1.0 & 0.506668 & 0.571486 & 0.481500 & Biso $1.000000 \mathrm{Ni}$ \\
\hline Mo1 & 1.0 & 0.569501 & 0.210644 & 0.303268 & Biso $1.000000 \mathrm{Mo}$ \\
\hline Mo2 & 1.0 & 0.553590 & 0.203930 & 0.057550 & Biso $1.000000 \mathrm{Mo}$ \\
\hline \multirow{2}{*}{$\begin{array}{l}\text { Mo3 } \\
\text { Mo4 }\end{array}$} & 1.0 & 0.775967 & 0.287495 & 0.387782 & Biso $1.000000 \mathrm{Mo}$ \\
\hline & 1.0 & 0.755790 & 0.280730 & 0.140960 & Biso $1.000000 \mathrm{Mo}$ \\
\hline Mo5 & 1.0 & 0.434016 & 0.379575 & 0.353437 & Biso $1.000000 \mathrm{Mo}$ \\
\hline Mo6 & 1.0 & 0.412350 & 0.376210 & 0.105210 & Biso $1.000000 \mathrm{Mo}$ \\
\hline Mo7 & 1.0 & 0.410837 & 0.269797 & 0.445011 & Biso $1.000000 \mathrm{Mo}$ \\
\hline Mo8 & 1.0 & 0.376910 & 0.261330 & 0.199500 & Biso $1.000000 \mathrm{Mo}$ \\
\hline Mo9 & 1.0 & 0.199471 & 0.197890 & 0.362548 & Biso $1.000000 \mathrm{Mo}$ \\
\hline Mo10 & 1.0 & 0.174710 & 0.184520 & 0.116090 & Biso $1.000000 \mathrm{Mo}$ \\
\hline Mo11 & 1.0 & 0.539699 & 0.106574 & 0.399493 & Biso $1.000000 \mathrm{Mo}$ \\
\hline Mo12 & 1.0 & 0.518150 & 0.089050 & 0.151830 & Biso $1.000000 \mathrm{Mo}$ \\
\hline Mo13 & 1.0 & 0.572720 & 0.716697 & 0.301337 & Biso $1.000000 \mathrm{Mo}$ \\
\hline Mo14 & 1.0 & 0.553590 & 0.703930 & 0.057550 & Biso $1.000000 \mathrm{Mo}$ \\
\hline Mo15 & 1.0 & 0.773569 & 0.785171 & 0.387476 & Biso $1.000000 \mathrm{Mo}$ \\
\hline Mo16 & 1.0 & 0.755790 & 0.780730 & 0.140960 & Biso $1.000000 \mathrm{Mo}$ \\
\hline Mo17 & 1.0 & 0.434627 & 0.881582 & 0.355865 & Biso $1.000000 \mathrm{Mo}$ \\
\hline Mo18 & 1.0 & 0.412350 & 0.876210 & 0.105210 & Biso $1.000000 \mathrm{Mo}$ \\
\hline Mo19 & 1.0 & 0.417679 & 0.767366 & 0.446859 & Biso $1.000000 \mathrm{Mo}$ \\
\hline Mo20 & 1.0 & 0.376910 & 0.761330 & 0.199500 & Biso $1.000000 \mathrm{Mo}$ \\
\hline Mo21 & 1.0 & 0.211813 & 0.697025 & 0.362899 & Biso $1.000000 \mathrm{Mo}$ \\
\hline Mo22 & 1.0 & 0.174710 & 0.684520 & 0.116090 & Biso $1.000000 \mathrm{Mo}$ \\
\hline Mo23 & 1.0 & 0.553081 & 0.606972 & 0.392135 & Biso $1.000000 \mathrm{Mo}$ \\
\hline Mo24 & 1.0 & 0.518150 & 0.589050 & 0.151830 & Biso $1.000000 \mathrm{Mo}$ \\
\hline S1 & 1.0 & 0.850160 & 0.122594 & 0.346088 & Biso $1.000000 \mathrm{~S}$ \\
\hline S2 & 1.0 & 0.830550 & 0.112740 & 0.100790 & Biso $1.000000 \mathrm{~S}$ \\
\hline S3 & 1.0 & 0.603173 & 0.433738 & 0.436409 & Biso $1.000000 \mathrm{~S}$ \\
\hline S4 & 1.0 & 0.583830 & 0.419330 & 0.186690 & Biso $1.000000 \mathrm{~S}$ \\
\hline S5 & 1.0 & 0.239719 & 0.295239 & 0.284121 & Biso $1.000000 \mathrm{~S}$ \\
\hline S6 & 1.0 & 0.219570 & 0.284900 & 0.038280 & Biso $1.000000 \mathrm{~S}$ \\
\hline
\end{tabular}




\begin{tabular}{|c|c|c|c|c|c|}
\hline S7 & 1.0 & 0.120635 & 0.362307 & 0.407704 & Biso $1.000000 \mathrm{~S}$ \\
\hline S8 & 1.0 & 0.099960 & 0.352520 & 0.156260 & Biso $1.000000 \mathrm{~S}$ \\
\hline S9 & 1.0 & 0.361258 & 0.052874 & 0.318679 & Biso $1.000000 \mathrm{~S}$ \\
\hline S10 & 1.0 & 0.346680 & 0.045920 & 0.070350 & Biso $1.000000 \mathrm{~S}$ \\
\hline S11 & 1.0 & 0.733176 & 0.198560 & 0.469881 & Biso $1.000000 \mathrm{~S}$ \\
\hline S12 & 1.0 & 0.710930 & 0.180350 & 0.218760 & Biso $1.000000 \mathrm{~S}$ \\
\hline S13 & 1.0 & 0.763855 & 0.374094 & 0.305942 & Biso $1.000000 \mathrm{~S}$ \\
\hline S14 & 1.0 & 0.740810 & 0.370400 & 0.059330 & Biso $1.000000 \mathrm{~S}$ \\
\hline S15 & 1.0 & 0.204374 & 0.114691 & 0.445760 & Biso $1.000000 \mathrm{~S}$ \\
\hline S16 & 1.0 & 0.189690 & 0.094850 & 0.197710 & Biso $1.000000 \mathrm{~S}$ \\
\hline S17 & 1.0 & 0.858424 & 0.623154 & 0.340593 & Biso $1.000000 \mathrm{~S}$ \\
\hline S18 & 1.0 & 0.830550 & 0.612740 & 0.100790 & Biso $1.000000 \mathrm{~S}$ \\
\hline S19 & 1.0 & 0.610747 & 0.932519 & 0.435114 & Biso $1.000000 \mathrm{~S}$ \\
\hline S20 & 1.0 & 0.583830 & 0.919330 & 0.186690 & Biso $1.000000 \mathrm{~S}$ \\
\hline S21 & 1.0 & 0.241525 & 0.795721 & 0.284924 & Biso $1.000000 \mathrm{~S}$ \\
\hline S22 & 1.0 & 0.219570 & 0.784900 & 0.038280 & Biso $1.000000 \mathrm{~S}$ \\
\hline S23 & 1.0 & 0.123445 & 0.859850 & 0.410639 & Biso $1.000000 \mathrm{~S}$ \\
\hline S24 & 1.0 & 0.099960 & 0.852520 & 0.156260 & Biso $1.000000 \mathrm{~S}$ \\
\hline S25 & 1.0 & 0.368275 & 0.553741 & 0.315409 & Biso $1.000000 \mathrm{~S}$ \\
\hline S26 & 1.0 & 0.346680 & 0.545920 & 0.070350 & Biso $1.000000 \mathrm{~S}$ \\
\hline S27 & 1.0 & 0.741211 & 0.697879 & 0.473156 & Biso $1.000000 \mathrm{~S}$ \\
\hline S28 & 1.0 & 0.710930 & 0.680350 & 0.218760 & Biso $1.000000 \mathrm{~S}$ \\
\hline S29 & 1.0 & 0.762966 & 0.879054 & 0.307608 & Biso $1.000000 \mathrm{~S}$ \\
\hline S30 & 1.0 & 0.740810 & 0.870400 & 0.059330 & Biso $1.000000 \mathrm{~S}$ \\
\hline S31 & 1.0 & 0.203292 & 0.614260 & 0.448704 & Biso $1.000000 \mathrm{~S}$ \\
\hline S32 & 1.0 & 0.189690 & 0.594850 & 0.197710 & Biso $1.000000 \mathrm{~S}$ \\
\hline
\end{tabular}

data_VESTA_phase_1

_chemical_name_common

\section{$\mathrm{C}_{2} \mathrm{H}_{2} *-\mathrm{Ni}_{1} \mathrm{MoS}$}

_cell_length_a

6.410100

12.820300

_cell_length_b

26.121099

_cell_length_c

90.000000

90.000000

_cell_angle_beta

91.297401

_cell_angle_gamma

2146.066112

_space_group_name_H-M_alt

'P 1'

_space_group_IT_number

1

loop_

_space_group_symop_operation_xyz

'x, y, z'

loop_

_atom_site_label 


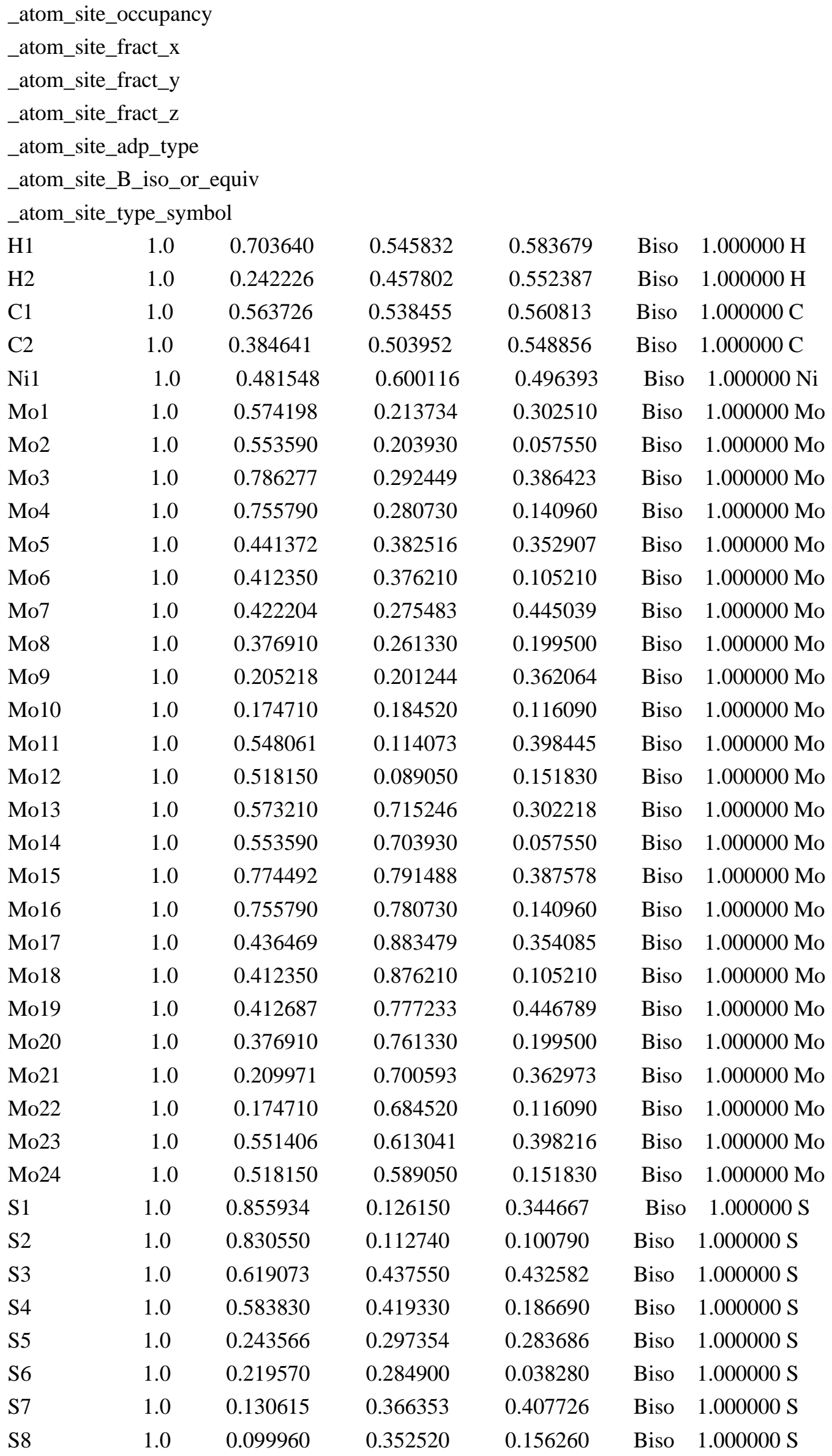




$\begin{array}{lllllll}\text { S9 } & 1.0 & 0.364636 & 0.056549 & 0.317999 & \text { Biso } & 1.000000 \mathrm{~S} \\ \text { S10 } & 1.0 & 0.346680 & 0.045920 & 0.070350 & \text { Biso } & 1.000000 \mathrm{~S} \\ \text { S11 } & 1.0 & 0.744565 & 0.206061 & 0.469123 & \text { Biso } & 1.000000 \mathrm{~S} \\ \text { S12 } & 1.0 & 0.710930 & 0.180350 & 0.218760 & \text { Biso } & 1.000000 \mathrm{~S} \\ \text { S13 } & 1.0 & 0.769987 & 0.377619 & 0.304575 & \text { Biso } & 1.000000 \mathrm{~S} \\ \text { S14 } & 1.0 & 0.740810 & 0.370400 & 0.059330 & \text { Biso } & 1.000000 \mathrm{~S} \\ \text { S15 } & 1.0 & 0.211494 & 0.122193 & 0.445757 & \text { Biso } & 1.000000 \mathrm{~S} \\ \text { S16 } & 1.0 & 0.189690 & 0.094850 & 0.197710 & \text { Biso } & 1.000000 \mathrm{~S} \\ \text { S17 } & 1.0 & 0.854929 & 0.625999 & 0.344410 & \text { Biso } & 1.000000 \mathrm{~S} \\ \text { S18 } & 1.0 & 0.830550 & 0.612740 & 0.100790 & \text { Biso } & 1.000000 \mathrm{~S} \\ \text { S19 } & 1.0 & 0.610580 & 0.939642 & 0.433487 & \text { Biso } & 1.000000 \mathrm{~S} \\ \text { S20 } & 1.0 & 0.583830 & 0.919330 & 0.186690 & \text { Biso } & 1.000000 \mathrm{~S} \\ \text { S21 } & 1.0 & 0.242458 & 0.796481 & 0.284271 & \text { Biso } & 1.000000 \mathrm{~S} \\ \text { S22 } & 1.0 & 0.219570 & 0.784900 & 0.038280 & \text { Biso } & 1.000000 \mathrm{~S} \\ \text { S23 } & 1.0 & 0.123182 & 0.867348 & 0.408261 & \text { Biso } & 1.000000 \mathrm{~S} \\ \text { S24 } & 1.0 & 0.099960 & 0.852520 & 0.156260 & \text { Biso } & 1.000000 \mathrm{~S} \\ \text { S25 } & 1.0 & 0.370444 & 0.556764 & 0.318919 & \text { Biso } & 1.000000 \mathrm{~S} \\ \text { S26 } & 1.0 & 0.346680 & 0.545920 & 0.070350 & \text { Biso } & 1.000000 \mathrm{~S} \\ \text { S27 } & 1.0 & 0.737563 & 0.711773 & 0.474307 & \text { Biso } & 1.000000 \mathrm{~S} \\ \text { S28 } & 1.0 & 0.710930 & 0.680350 & 0.218760 & \text { Biso } & 1.000000 \mathrm{~S} \\ \text { S29 } & 1.0 & 0.764539 & 0.879094 & 0.306448 & \text { Biso } & 1.000000 \mathrm{~S} \\ \text { S30 } & 1.0 & 0.740810 & 0.870400 & 0.059330 & \text { Biso } & 1.000000 \mathrm{~S} \\ \text { S31 } & 1.0 & 0.197608 & 0.624552 & 0.449359 & \text { Biso } & 1.000000 \mathrm{~S} \\ \text { S32 } & 1.0 & 0.189690 & 0.594850 & 0.197710 & \text { Biso } & 1.000000 \mathrm{~S}\end{array}$

data_VESTA_phase_1

_chemical_name_common

$\mathrm{C}_{2} \mathrm{H}_{2}{ }^{*}+\mathrm{H}_{2}-\mathrm{Ni}_{1} \mathrm{MoS}$

_cell_length_a

6.410100

12.820300

26.121099

_cell_length_c

90.000000

_cell_angle_alpha

90.000000

_cell_angle_beta

91.297401

_cell_angle_gamma

2146.066112

_cell_volume

'P 1'

_space_group_name_H-M_alt

1

loop_

_space_group_symop_operation_xyz

'x, y, z'

loop_

_atom_site_label

_atom_site_occupancy

_atom_site_fract_x 


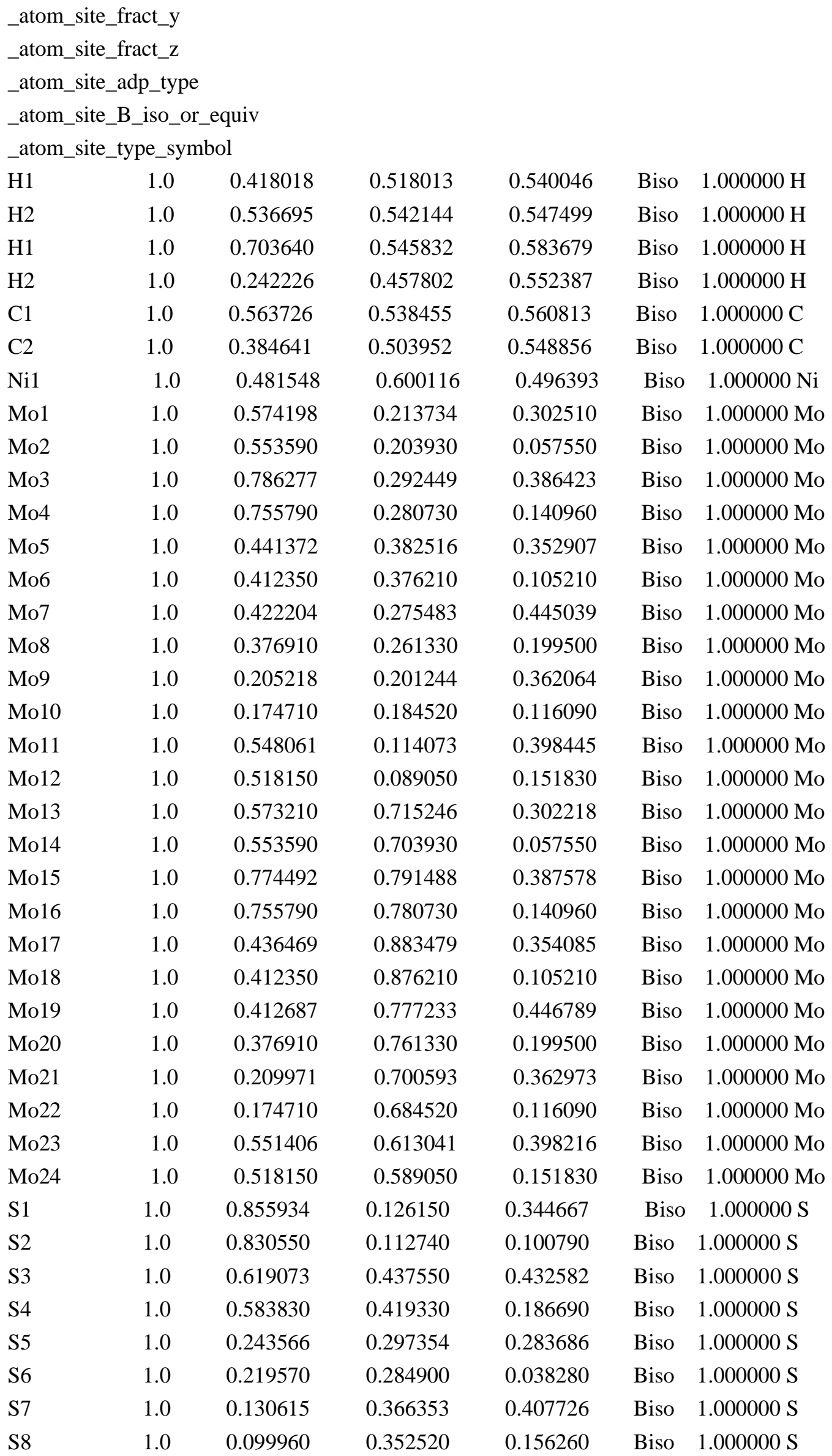




$\begin{array}{lllllll}\text { S9 } & 1.0 & 0.364636 & 0.056549 & 0.317999 & \text { Biso } & 1.000000 \mathrm{~S} \\ \text { S10 } & 1.0 & 0.346680 & 0.045920 & 0.070350 & \text { Biso } & 1.000000 \mathrm{~S} \\ \text { S11 } & 1.0 & 0.744565 & 0.206061 & 0.469123 & \text { Biso } & 1.000000 \mathrm{~S} \\ \text { S12 } & 1.0 & 0.710930 & 0.180350 & 0.218760 & \text { Biso } & 1.000000 \mathrm{~S} \\ \text { S13 } & 1.0 & 0.769987 & 0.377619 & 0.304575 & \text { Biso } & 1.000000 \mathrm{~S} \\ \text { S14 } & 1.0 & 0.740810 & 0.370400 & 0.059330 & \text { Biso } & 1.000000 \mathrm{~S} \\ \text { S15 } & 1.0 & 0.211494 & 0.122193 & 0.445757 & \text { Biso } & 1.000000 \mathrm{~S} \\ \text { S16 } & 1.0 & 0.189690 & 0.094850 & 0.197710 & \text { Biso } & 1.000000 \mathrm{~S} \\ \text { S17 } & 1.0 & 0.854929 & 0.625999 & 0.344410 & \text { Biso } & 1.000000 \mathrm{~S} \\ \text { S18 } & 1.0 & 0.830550 & 0.612740 & 0.100790 & \text { Biso } & 1.000000 \mathrm{~S} \\ \text { S19 } & 1.0 & 0.610580 & 0.939642 & 0.433487 & \text { Biso } & 1.000000 \mathrm{~S} \\ \text { S20 } & 1.0 & 0.583830 & 0.919330 & 0.186690 & \text { Biso } & 1.000000 \mathrm{~S} \\ \text { S21 } & 1.0 & 0.242458 & 0.796481 & 0.284271 & \text { Biso } & 1.000000 \mathrm{~S} \\ \text { S22 } & 1.0 & 0.219570 & 0.784900 & 0.038280 & \text { Biso } & 1.000000 \mathrm{~S} \\ \text { S23 } & 1.0 & 0.123182 & 0.867348 & 0.408261 & \text { Biso } & 1.000000 \mathrm{~S} \\ \text { S24 } & 1.0 & 0.099960 & 0.852520 & 0.156260 & \text { Biso } & 1.000000 \mathrm{~S} \\ \text { S25 } & 1.0 & 0.370444 & 0.556764 & 0.318919 & \text { Biso } & 1.000000 \mathrm{~S} \\ \text { S26 } & 1.0 & 0.346680 & 0.545920 & 0.070350 & \text { Biso } & 1.000000 \mathrm{~S} \\ \text { S27 } & 1.0 & 0.737563 & 0.711773 & 0.474307 & \text { Biso } & 1.000000 \mathrm{~S} \\ \text { S28 } & 1.0 & 0.710930 & 0.680350 & 0.218760 & \text { Biso } & 1.000000 \mathrm{~S} \\ \text { S29 } & 1.0 & 0.764539 & 0.879094 & 0.306448 & \text { Biso } & 1.000000 \mathrm{~S} \\ \text { S30 } & 1.0 & 0.740810 & 0.870400 & 0.059330 & \text { Biso } & 1.000000 \mathrm{~S} \\ \text { S31 } & 1.0 & 0.197608 & 0.624552 & 0.449359 & \text { Biso } & 1.000000 \mathrm{~S} \\ \text { S32 } & 1.0 & 0.189690 & 0.594850 & 0.197710 & \text { Biso } & 1.000000 \mathrm{~S}\end{array}$

data_VESTA_phase_1

_chemical_name_common

TS1

_cell_length_a

6.410100

12.820300

_cell_length_b

26.121099

_cell_length_c

90.000000

_cell_angle_beta

90.000000

91.297401

_cell_angle_gamma

2146.066112

_cell_volume

'P 1'

_space_group_name_H-M_alt

1

loop_

_space_group_symop_operation_xyz

'x, y, z'

loop_

_atom_site_label

_atom_site_occupancy

_atom_site_fract_x 


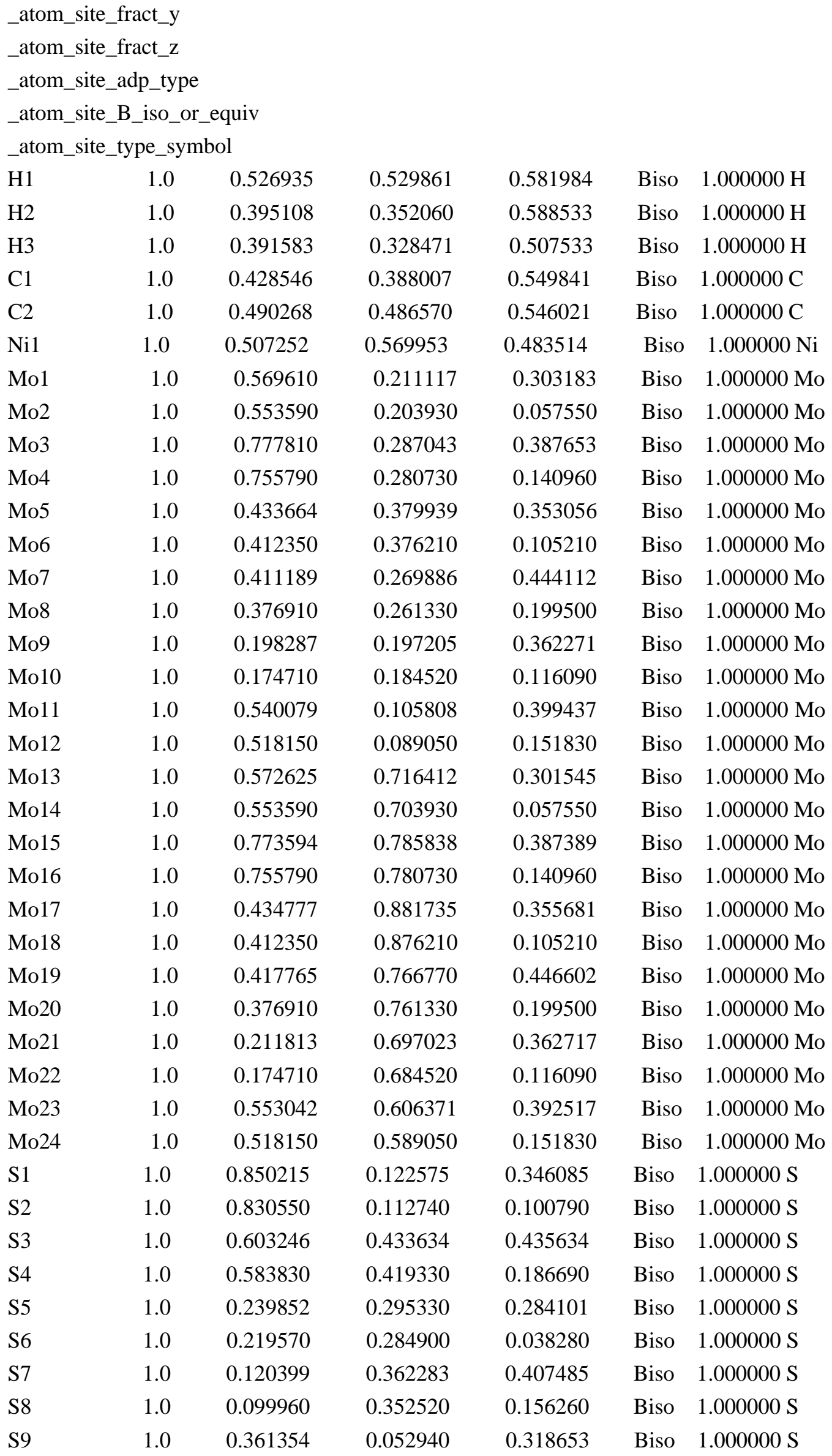




$\begin{array}{lllllll}\text { S10 } & 1.0 & 0.346680 & 0.045920 & 0.070350 & \text { Biso } & 1.000000 \mathrm{~S} \\ \text { S11 } & 1.0 & 0.734579 & 0.198435 & 0.469494 & \text { Biso } & 1.000000 \mathrm{~S} \\ \text { S12 } & 1.0 & 0.710930 & 0.180350 & 0.218760 & \text { Biso } & 1.000000 \mathrm{~S} \\ \text { S13 } & 1.0 & 0.763744 & 0.374366 & 0.305901 & \text { Biso } & 1.000000 \mathrm{~S} \\ \text { S14 } & 1.0 & 0.740810 & 0.370400 & 0.059330 & \text { Biso } & 1.000000 \mathrm{~S} \\ \text { S15 } & 1.0 & 0.203784 & 0.114498 & 0.445502 & \text { Biso } & 1.000000 \mathrm{~S} \\ \text { S16 } & 1.0 & 0.189690 & 0.094850 & 0.197710 & \text { Biso } & 1.000000 \mathrm{~S} \\ \text { S17 } & 1.0 & 0.858817 & 0.623270 & 0.340636 & \text { Biso } & 1.000000 \mathrm{~S} \\ \text { S18 } & 1.0 & 0.830550 & 0.612740 & 0.100790 & \text { Biso } & 1.000000 \mathrm{~S} \\ \text { S19 } & 1.0 & 0.610602 & 0.932577 & 0.435118 & \text { Biso } & 1.000000 \mathrm{~S} \\ \text { S20 } & 1.0 & 0.583830 & 0.919330 & 0.186690 & \text { Biso } & 1.000000 \mathrm{~S} \\ \text { S21 } & 1.0 & 0.241343 & 0.796027 & 0.284896 & \text { Biso } & 1.000000 \mathrm{~S} \\ \text { S22 } & 1.0 & 0.219570 & 0.784900 & 0.038280 & \text { Biso } & 1.000000 \mathrm{~S} \\ \text { S23 } & 1.0 & 0.123589 & 0.859752 & 0.410518 & \text { Biso } & 1.000000 \mathrm{~S} \\ \text { S24 } & 1.0 & 0.099960 & 0.852520 & 0.156260 & \text { Biso } & 1.000000 \mathrm{~S} \\ \text { S25 } & 1.0 & 0.368132 & 0.553648 & 0.315226 & \text { Biso } & 1.000000 \mathrm{~S} \\ \text { S26 } & 1.0 & 0.346680 & 0.545920 & 0.070350 & \text { Biso } & 1.000000 \mathrm{~S} \\ \text { S27 } & 1.0 & 0.741402 & 0.697748 & 0.472710 & \text { Biso } & 1.000000 \mathrm{~S} \\ \text { S28 } & 1.0 & 0.710930 & 0.680350 & 0.218760 & \text { Biso } & 1.000000 \mathrm{~S} \\ \text { S29 } & 1.0 & 0.763084 & 0.879493 & 0.307627 & \text { Biso } & 1.000000 \mathrm{~S} \\ \text { S30 } & 1.0 & 0.740810 & 0.870400 & 0.059330 & \text { Biso } & 1.000000 \mathrm{~S} \\ \text { S31 } & 1.0 & 0.203624 & 0.614066 & 0.448472 & \text { Biso } & 1.000000 \mathrm{~S} \\ \text { S32 } & 1.0 & 0.189690 & 0.594850 & 0.197710 & \text { Biso } & 1.000000 \mathrm{~S}\end{array}$

data_VESTA_phase_1

$\begin{array}{ll}\text { _chemical_name_common } & \mathbf{C}_{2} \mathbf{H}_{3}{ }^{*}-\mathbf{N i}_{1} \mathbf{M o S} \\ \text { _cell_length_a } & 6.410100 \\ \text { _cell_length_b } & 12.820300 \\ \text { _cell_length_c } & 26.121099 \\ \text { _cell_angle_alpha } & 90.000000 \\ \text { _cell_angle_beta } & 90.000000 \\ \text { _cell_angle_gamma } & 91.297401 \\ \text { _cell_volume } & 2146.066112 \\ \text { _space_group_name_H-M_alt } & \text { P 1' } \\ \text { _space_group_IT_number } & 1 \\ \text { loop_ } & \\ \text { _space_group_symop_operation_xyz } & \\ \quad \text { 'x, y, z' } & \\ \text { loop_ } & \\ \text { _atom_site_label } & \\ \text { _atom_site_occupancy } & \\ \quad \text { atom_site_fract_x } & \\ \quad \text { atom_site_fract_y } & \end{array}$




\begin{tabular}{|c|c|c|c|c|c|c|}
\hline \multicolumn{7}{|c|}{$\begin{array}{l}\text { _atom_site_adp_type } \\
\text { _atom_site_B_iso_or_equiv } \\
\text { _atom_site_type_symbol }\end{array}$} \\
\hline $\mathrm{H} 1$ & 1.0 & 0.588154 & 0.389441 & 0.538978 & Biso & $1.000000 \mathrm{H}$ \\
\hline $\mathrm{H} 2$ & 1.0 & 0.277654 & 0.280947 & 0.547117 & Biso & $1.000000 \mathrm{H}$ \\
\hline H3 & 1.0 & 0.120638 & 0.373081 & 0.509277 & Biso & $1.000000 \mathrm{H}$ \\
\hline $\mathrm{C} 1$ & 1.0 & 0.274317 & 0.350884 & 0.522918 & Biso & $1.000000 \mathrm{C}$ \\
\hline $\mathrm{C} 2$ & 1.0 & 0.445135 & 0.416916 & 0.520294 & Biso & $1.000000 \mathrm{C}$ \\
\hline Ni1 & 1.0 & 0.494256 & 0.543891 & 0.483276 & Biso & $1.000000 \mathrm{Ni}$ \\
\hline Mo1 & 1.0 & 0.574989 & 0.213244 & 0.304629 & Biso & $1.000000 \mathrm{Mo}$ \\
\hline Mo2 & 1.0 & 0.553590 & 0.203930 & 0.057550 & Biso & $1.000000 \mathrm{Mo}$ \\
\hline Mo3 & 1.0 & 0.784180 & 0.290674 & 0.386505 & Biso & $1.000000 \mathrm{Mo}$ \\
\hline Mo4 & 1.0 & 0.755790 & 0.280730 & 0.140960 & Biso & $1.000000 \mathrm{Mo}$ \\
\hline Mo5 & 1.0 & 0.437697 & 0.387141 & 0.347079 & Biso & $1.000000 \mathrm{Mo}$ \\
\hline Mo6 & 1.0 & 0.412350 & 0.376210 & 0.105210 & Biso & $1.000000 \mathrm{Mo}$ \\
\hline Mo7 & 1.0 & 0.422728 & 0.281174 & 0.438960 & Biso & $1.000000 \mathrm{Mo}$ \\
\hline Mo8 & 1.0 & 0.376910 & 0.261330 & 0.199500 & Biso & $1.000000 \mathrm{Mo}$ \\
\hline Mo9 & 1.0 & 0.201161 & 0.196849 & 0.362822 & Biso & $1.000000 \mathrm{Mo}$ \\
\hline Mo10 & 1.0 & 0.174710 & 0.184520 & 0.116090 & Biso & $1.000000 \mathrm{Mo}$ \\
\hline Mo11 & 1.0 & 0.544371 & 0.101562 & 0.402316 & Biso & $1.000000 \mathrm{Mo}$ \\
\hline Mo12 & 1.0 & 0.518150 & 0.089050 & 0.151830 & Biso & $1.000000 \mathrm{Mo}$ \\
\hline Mo13 & 1.0 & 0.569125 & 0.718368 & 0.301704 & Biso & $1.000000 \mathrm{Mo}$ \\
\hline Mo14 & 1.0 & 0.553590 & 0.703930 & 0.057550 & Biso & $1.000000 \mathrm{Mo}$ \\
\hline Mo15 & 1.0 & 0.764146 & 0.787875 & 0.387804 & Biso & $1.000000 \mathrm{Mo}$ \\
\hline Mo16 & 1.0 & 0.755790 & 0.780730 & 0.140960 & Biso & $1.000000 \mathrm{Mo}$ \\
\hline Mo17 & 1.0 & 0.428870 & 0.884288 & 0.356160 & Biso & $1.000000 \mathrm{Mo}$ \\
\hline Mo18 & 1.0 & 0.412350 & 0.876210 & 0.105210 & Biso & $1.000000 \mathrm{Mo}$ \\
\hline Mo19 & 1.0 & 0.397892 & 0.760694 & 0.445640 & Biso & $1.000000 \mathrm{Mo}$ \\
\hline Mo20 & 1.0 & 0.376910 & 0.761330 & 0.199500 & Biso & $1.000000 \mathrm{Mo}$ \\
\hline Mo21 & 1.0 & 0.202479 & 0.699185 & 0.359934 & Biso & $1.000000 \mathrm{Mo}$ \\
\hline Mo22 & 1.0 & 0.174710 & 0.684520 & 0.116090 & Biso & $1.000000 \mathrm{Mo}$ \\
\hline Mo23 & 1.0 & 0.541366 & 0.607015 & 0.390525 & Biso & $1.000000 \mathrm{Mo}$ \\
\hline Mo24 & 1.0 & 0.518150 & 0.589050 & 0.151830 & Biso & $1.000000 \mathrm{Mo}$ \\
\hline $\mathrm{S} 1$ & 1.0 & 0.854071 & 0.121151 & 0.349113 & Biso & $1.000000 \mathrm{~S}$ \\
\hline S2 & 1.0 & 0.830550 & 0.112740 & 0.100790 & Biso & $1.000000 \mathrm{~S}$ \\
\hline S3 & 1.0 & 0.630660 & 0.439837 & 0.424135 & Biso & $1.000000 \mathrm{~S}$ \\
\hline S4 & 1.0 & 0.583830 & 0.419330 & 0.186690 & Biso & $1.000000 \mathrm{~S}$ \\
\hline S5 & 1.0 & 0.236789 & 0.294249 & 0.283010 & Biso & $1.000000 \mathrm{~S}$ \\
\hline S6 & 1.0 & 0.219570 & 0.284900 & 0.038280 & Biso & $1.000000 \mathrm{~S}$ \\
\hline S7 & 1.0 & 0.129657 & 0.365322 & 0.399424 & Biso & $1.000000 \mathrm{~S}$ \\
\hline S8 & 1.0 & 0.099960 & 0.352520 & 0.156260 & Biso & $1.000000 \mathrm{~S}$ \\
\hline S9 & 1.0 & 0.372114 & 0.055116 & 0.319902 & Biso & $1.000000 \mathrm{~S}$ \\
\hline S10 & 1.0 & 0.346680 & 0.045920 & 0.070350 & Biso & $1.000000 \mathrm{~S}$ \\
\hline
\end{tabular}




$\begin{array}{lllllll}\text { S11 } & 1.0 & 0.743020 & 0.194899 & 0.466895 & \text { Biso } & 1.000000 \mathrm{~S} \\ \text { S12 } & 1.0 & 0.710930 & 0.180350 & 0.218760 & \text { Biso } & 1.000000 \mathrm{~S} \\ \text { S13 } & 1.0 & 0.769099 & 0.380843 & 0.303973 & \text { Biso } & 1.000000 \mathrm{~S} \\ \text { S14 } & 1.0 & 0.740810 & 0.370400 & 0.059330 & \text { Biso } & 1.000000 \mathrm{~S} \\ \text { S15 } & 1.0 & 0.219918 & 0.117142 & 0.446732 & \text { Biso } & 1.000000 \mathrm{~S} \\ \text { S16 } & 1.0 & 0.189690 & 0.094850 & 0.197710 & \text { Biso } & 1.000000 \mathrm{~S} \\ \text { S17 } & 1.0 & 0.852879 & 0.626434 & 0.340561 & \text { Biso } & 1.000000 \mathrm{~S} \\ \text { S18 } & 1.0 & 0.830550 & 0.612740 & 0.100790 & \text { Biso } & 1.000000 \mathrm{~S} \\ \text { S19 } & 1.0 & 0.592145 & 0.926756 & 0.438532 & \text { Biso } & 1.000000 \mathrm{~S} \\ \text { S20 } & 1.0 & 0.583830 & 0.919330 & 0.186690 & \text { Biso } & 1.000000 \mathrm{~S} \\ \text { S21 } & 1.0 & 0.238594 & 0.803040 & 0.284134 & \text { Biso } & 1.000000 \mathrm{~S} \\ \text { S22 } & 1.0 & 0.219570 & 0.784900 & 0.038280 & \text { Biso } & 1.000000 \mathrm{~S} \\ \text { S23 } & 1.0 & 0.114239 & 0.860682 & 0.409262 & \text { Biso } & 1.000000 \mathrm{~S} \\ \text { S24 } & 1.0 & 0.099960 & 0.852520 & 0.156260 & \text { Biso } & 1.000000 \mathrm{~S} \\ \text { S25 } & 1.0 & 0.358285 & 0.558915 & 0.311564 & \text { Biso } & 1.000000 \mathrm{~S} \\ \text { S26 } & 1.0 & 0.346680 & 0.545920 & 0.070350 & \text { Biso } & 1.000000 \mathrm{~S} \\ \text { S27 } & 1.0 & 0.721741 & 0.685330 & 0.469530 & \text { Biso } & 1.000000 \mathrm{~S} \\ \text { S28 } & 1.0 & 0.710930 & 0.680350 & 0.218760 & \text { Biso } & 1.000000 \mathrm{~S} \\ \text { S29 } & 1.0 & 0.755273 & 0.882630 & 0.308035 & \text { Biso } & 1.000000 \mathrm{~S} \\ \text { S30 } & 1.0 & 0.740810 & 0.870400 & 0.059330 & \text { Biso } & 1.000000 \mathrm{~S} \\ \text { S31 } & 1.0 & 0.200979 & 0.603523 & 0.445126 & \text { Biso } & 1.000000 \mathrm{~S} \\ \text { S32 } & 1.0 & 0.189690 & 0.594850 & 0.197710 & \text { Biso } & 1.000000 \mathrm{~S}\end{array}$

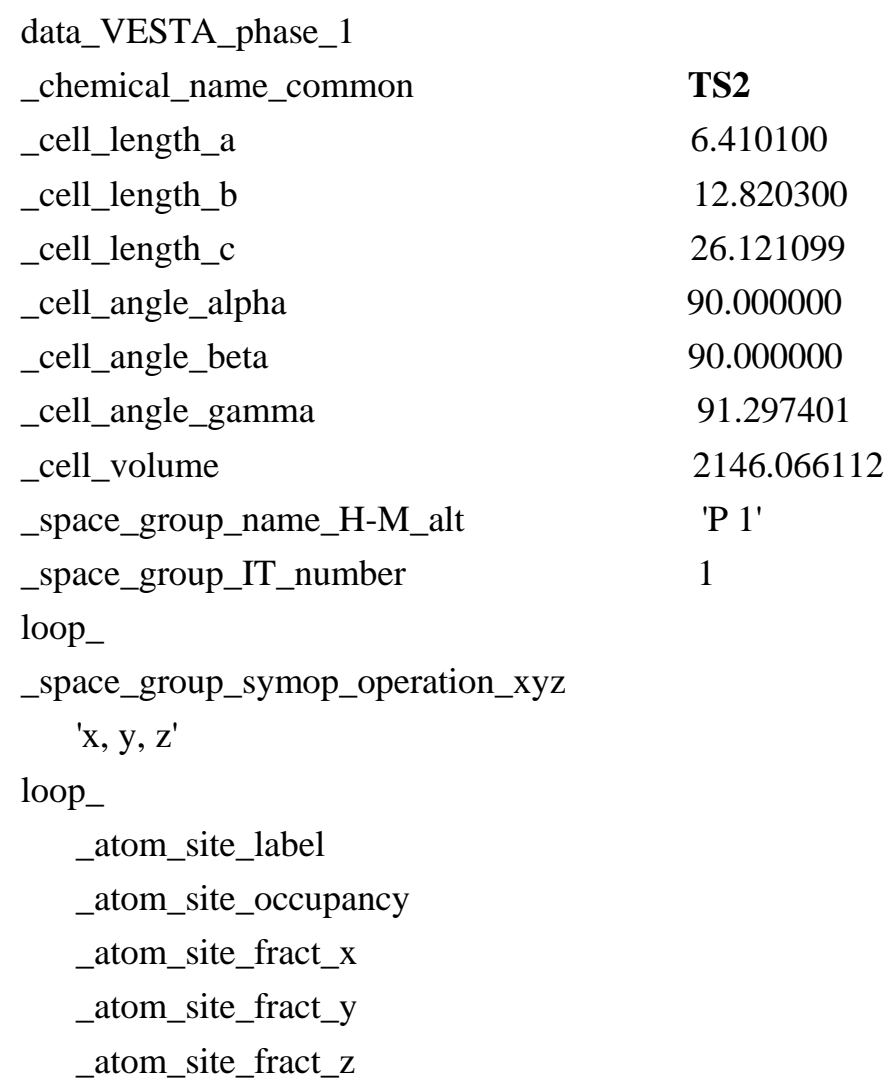

\section{TS2}

6.410100

12.820300

26.121099

90.000000

90.000000

91.297401

2146.066112

'P 1'

1 


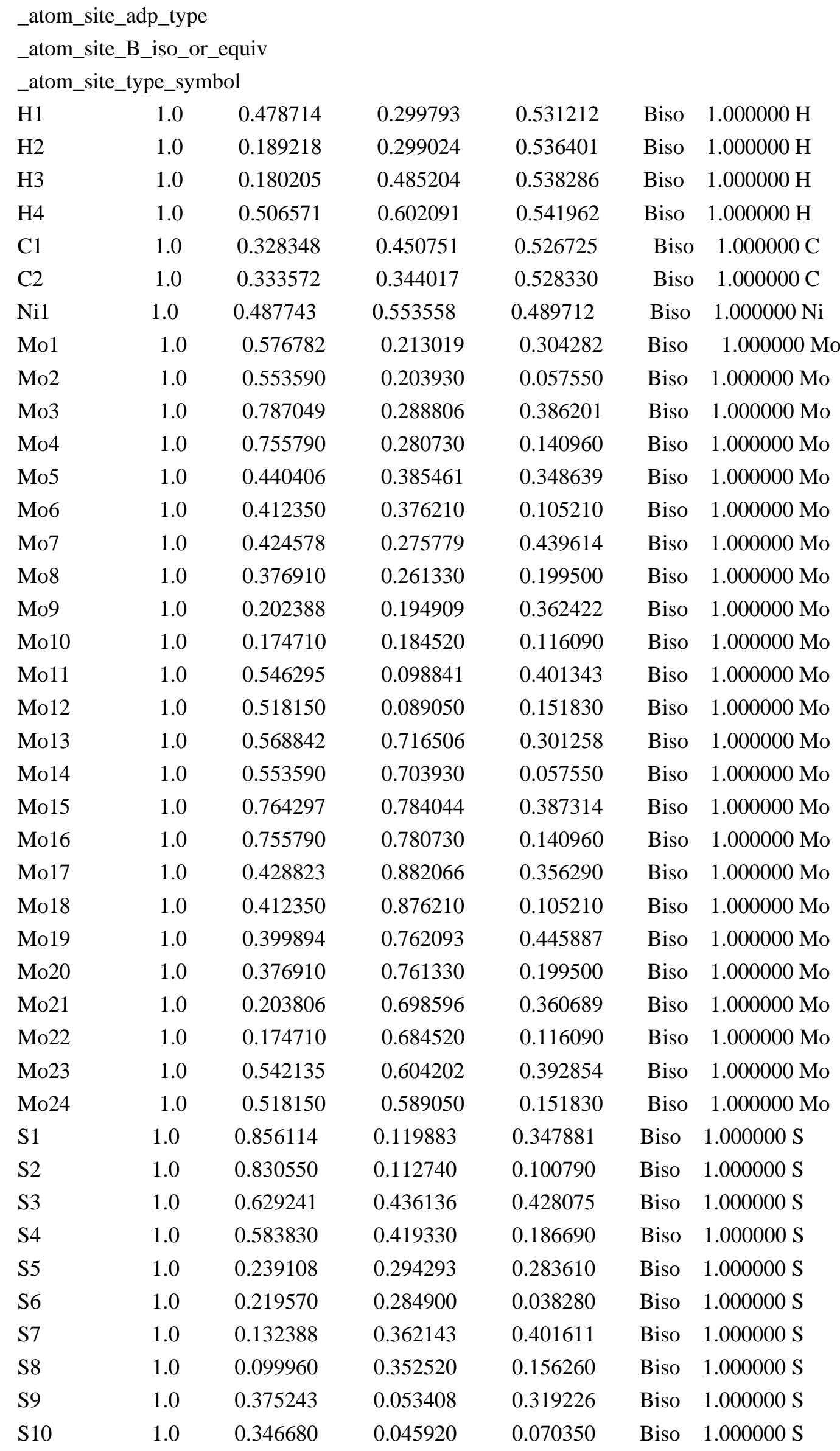




$\begin{array}{lllllll}\text { S11 } & 1.0 & 0.750709 & 0.191711 & 0.465727 & \text { Biso } 1.000000 \mathrm{~S} \\ \text { S12 } & 1.0 & 0.710930 & 0.180350 & 0.218760 & \text { Biso } & 1.000000 \mathrm{~S} \\ \text { S13 } & 1.0 & 0.770321 & 0.381188 & 0.304772 & \text { Biso } & 1.000000 \mathrm{~S} \\ \text { S14 } & 1.0 & 0.740810 & 0.370400 & 0.059330 & \text { Biso } & 1.000000 \mathrm{~S} \\ \text { S15 } & 1.0 & 0.221147 & 0.113099 & 0.445905 & \text { Biso } & 1.000000 \mathrm{~S} \\ \text { S16 } & 1.0 & 0.189690 & 0.094850 & 0.197710 & \text { Biso } & 1.000000 \mathrm{~S} \\ \text { S17 } & 1.0 & 0.851430 & 0.622999 & 0.340833 & \text { Biso } & 1.000000 \mathrm{~S} \\ \text { S18 } & 1.0 & 0.830550 & 0.612740 & 0.100790 & \text { Biso } & 1.000000 \mathrm{~S} \\ \text { S19 } & 1.0 & 0.597268 & 0.925789 & 0.437765 & \text { Biso } & 1.000000 \mathrm{~S} \\ \text { S20 } & 1.0 & 0.583830 & 0.919330 & 0.186690 & \text { Biso } & 1.000000 \mathrm{~S} \\ \text { S21 } & 1.0 & 0.239606 & 0.800738 & 0.284178 & \text { Biso } & 1.000000 \mathrm{~S} \\ \text { S22 } & 1.0 & 0.219570 & 0.784900 & 0.038280 & \text { Biso } & 1.000000 \mathrm{~S} \\ \text { S23 } & 1.0 & 0.113587 & 0.859651 & 0.410231 & \text { Biso } & 1.000000 \mathrm{~S} \\ \text { S24 } & 1.0 & 0.099960 & 0.852520 & 0.156260 & \text { Biso } & 1.000000 \mathrm{~S} \\ \text { S25 } & 1.0 & 0.359948 & 0.556298 & 0.312926 & \text { Biso } & 1.000000 \mathrm{~S} \\ \text { S26 } & 1.0 & 0.346680 & 0.545920 & 0.070350 & \text { Biso } & 1.000000 \mathrm{~S} \\ \text { S27 } & 1.0 & 0.723090 & 0.686593 & 0.470470 & \text { Biso } & 1.000000 \mathrm{~S} \\ \text { S28 } & 1.0 & 0.710930 & 0.680350 & 0.218760 & \text { Biso } & 1.000000 \mathrm{~S} \\ \text { S29 } & 1.0 & 0.757498 & 0.879275 & 0.308113 & \text { Biso } & 1.000000 \mathrm{~S} \\ \text { S30 } & 1.0 & 0.740810 & 0.870400 & 0.059330 & \text { Biso } & 1.000000 \mathrm{~S} \\ \text { S31 } & 1.0 & 0.196675 & 0.605495 & 0.442272 & \text { Biso } & 1.000000 \mathrm{~S} \\ \text { S32 } & 1.0 & 0.189690 & 0.594850 & 0.197710 & \text { Biso } 1.000000 \mathrm{~S}\end{array}$

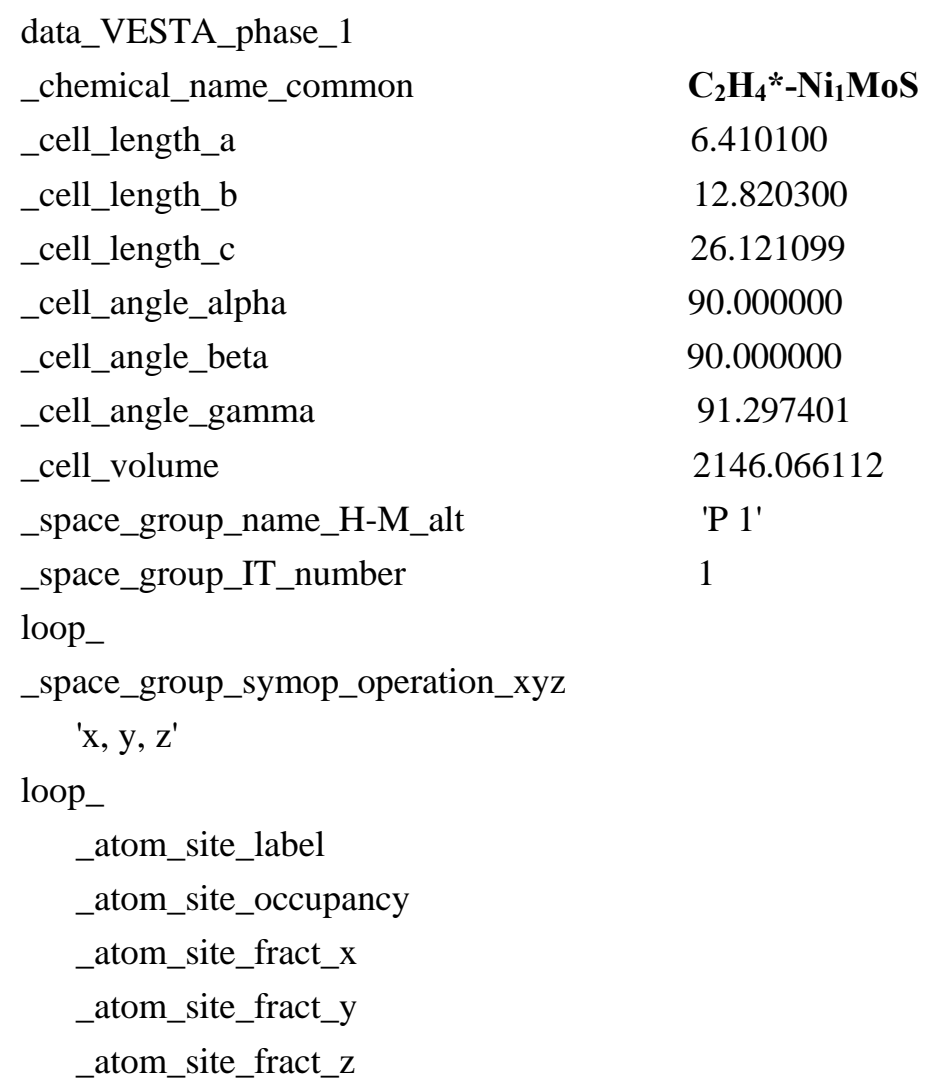

\section{$\mathrm{C}_{2} \mathrm{H}_{4} * \mathrm{Ni}_{1} \mathrm{MoS}$}

6.410100

12.820300

26.121099

90.000000

90.000000

91.297401

2146.066112

'P 1'

1 


\begin{tabular}{|c|c|c|c|c|c|c|}
\hline \multicolumn{7}{|c|}{ _atom_site_type_symbol } \\
\hline H1 & 1.0 & 0.690192 & 0.478292 & 0.564735 & Biso & $1.000000 \mathrm{H}$ \\
\hline $\mathrm{H} 2$ & 1.0 & 0.426909 & 0.420224 & 0.554043 & Biso & $1.000000 \mathrm{H}$ \\
\hline $\mathrm{H} 3$ & 1.0 & 0.267475 & 0.594362 & 0.567855 & Biso & $1.000000 \mathrm{H}$ \\
\hline $\mathrm{H} 4$ & 1.0 & 0.530338 & 0.653141 & 0.579410 & Biso & $1.000000 \mathrm{H}$ \\
\hline $\mathrm{C} 1$ & 1.0 & 0.436575 & 0.587035 & 0.565203 & Biso & $1.000000 \mathrm{C}$ \\
\hline $\mathrm{C} 2$ & 1.0 & 0.525007 & 0.490797 & 0.557222 & Biso & $1.000000 \mathrm{C}$ \\
\hline Ni1 & 1.0 & 0.512432 & 0.571444 & 0.488517 & Biso & $1.000000 \mathrm{Ni}$ \\
\hline Mo1 & 1.0 & 0.573351 & 0.214106 & 0.303204 & Biso & $1.000000 \mathrm{Mo}$ \\
\hline Mo2 & 1.0 & 0.553590 & 0.203930 & 0.057550 & Biso & $1.000000 \mathrm{Mo}$ \\
\hline Mo3 & 1.0 & 0.780172 & 0.292147 & 0.387708 & Biso & $1.000000 \mathrm{Mo}$ \\
\hline Mo4 & 1.0 & 0.755790 & 0.280730 & 0.140960 & Biso & $1.000000 \mathrm{Mo}$ \\
\hline Mo5 & 1.0 & 0.437619 & 0.383832 & 0.353169 & Biso & $1.000000 \mathrm{Mo}$ \\
\hline Mo6 & 1.0 & 0.412350 & 0.376210 & 0.105210 & Biso & $1.000000 \mathrm{Mo}$ \\
\hline Mo7 & 1.0 & 0.416080 & 0.274675 & 0.445335 & Biso & $1.000000 \mathrm{Mo}$ \\
\hline Mo8 & 1.0 & 0.376910 & 0.261330 & 0.199500 & Biso & $1.000000 \mathrm{Mo}$ \\
\hline Mo9 & 1.0 & 0.204653 & 0.201965 & 0.362605 & Biso & $1.000000 \mathrm{Mo}$ \\
\hline Mo10 & 1.0 & 0.174710 & 0.184520 & 0.116090 & Biso & $1.000000 \mathrm{Mo}$ \\
\hline Mo11 & 1.0 & 0.545432 & 0.112379 & 0.399173 & Biso & $1.000000 \mathrm{Mo}$ \\
\hline Mo12 & 1.0 & 0.518150 & 0.089050 & 0.151830 & Biso & $1.000000 \mathrm{Mo}$ \\
\hline Mo13 & 1.0 & 0.574392 & 0.718821 & 0.301155 & Biso & $1.000000 \mathrm{Mo}$ \\
\hline Mo14 & 1.0 & 0.553590 & 0.703930 & 0.057550 & Biso & $1.000000 \mathrm{Mo}$ \\
\hline Mo15 & 1.0 & 0.776825 & 0.789713 & 0.386896 & Biso & $1.000000 \mathrm{Mo}$ \\
\hline Mo16 & 1.0 & 0.755790 & 0.780730 & 0.140960 & Biso & $1.000000 \mathrm{Mo}$ \\
\hline Mo17 & 1.0 & 0.438762 & 0.884805 & 0.355202 & Biso & $1.000000 \mathrm{Mo}$ \\
\hline Mo18 & 1.0 & 0.412350 & 0.876210 & 0.105210 & Biso & $1.000000 \mathrm{Mo}$ \\
\hline Mo19 & 1.0 & 0.420401 & 0.772602 & 0.446259 & Biso & $1.000000 \mathrm{Mo}$ \\
\hline Mo20 & 1.0 & 0.376910 & 0.761330 & 0.199500 & Biso & $1.000000 \mathrm{Mo}$ \\
\hline Mo21 & 1.0 & 0.214757 & 0.701233 & 0.362878 & Biso & $1.000000 \mathrm{Mo}$ \\
\hline Mo22 & 1.0 & 0.174710 & 0.684520 & 0.116090 & Biso & $1.000000 \mathrm{Mo}$ \\
\hline Mo23 & 1.0 & 0.553762 & 0.610686 & 0.395074 & Biso & $1.000000 \mathrm{Mo}$ \\
\hline Mo24 & 1.0 & 0.518150 & 0.589050 & 0.151830 & Biso & $1.000000 \mathrm{Mo}$ \\
\hline $\mathrm{S} 1$ & 1.0 & 0.854973 & 0.127119 & 0.345693 & Biso & $1.000000 \mathrm{~S}$ \\
\hline $\mathrm{S} 2$ & 1.0 & 0.830550 & 0.112740 & 0.100790 & Biso & $1.000000 \mathrm{~S}$ \\
\hline S3 & 1.0 & 0.605610 & 0.438474 & 0.435642 & Biso & $1.000000 \mathrm{~S}$ \\
\hline S4 & 1.0 & 0.583830 & 0.419330 & 0.186690 & Biso & $1.000000 \mathrm{~S}$ \\
\hline S5 & 1.0 & 0.243541 & 0.298189 & 0.283949 & Biso & $1.000000 \mathrm{~S}$ \\
\hline S6 & 1.0 & 0.219570 & 0.284900 & 0.038280 & Biso & $1.000000 \mathrm{~S}$ \\
\hline S7 & 1.0 & 0.125289 & 0.366734 & 0.407842 & Biso & $1.000000 \mathrm{~S}$ \\
\hline S8 & 1.0 & 0.099960 & 0.352520 & 0.156260 & Biso & $1.000000 \mathrm{~S}$ \\
\hline S9 & 1.0 & 0.364732 & 0.056950 & 0.318491 & Biso & $1.000000 \mathrm{~S}$ \\
\hline $\mathrm{S} 10$ & 1.0 & 0.346680 & 0.045920 & 0.070350 & Biso & $1.000000 \mathrm{~S}$ \\
\hline
\end{tabular}




$\begin{array}{lllllll}\text { S11 } & 1.0 & 0.738556 & 0.204567 & 0.470098 & \text { Biso } & 1.000000 \mathrm{~S} \\ \text { S12 } & 1.0 & 0.710930 & 0.180350 & 0.218760 & \text { Biso } & 1.000000 \mathrm{~S} \\ \text { S13 } & 1.0 & 0.767537 & 0.377735 & 0.305557 & \text { Biso } & 1.000000 \mathrm{~S} \\ \text { S14 } & 1.0 & 0.740810 & 0.370400 & 0.059330 & \text { Biso } & 1.000000 \mathrm{~S} \\ \text { S15 } & 1.0 & 0.208878 & 0.120288 & 0.446015 & \text { Biso } & 1.000000 \mathrm{~S} \\ \text { S16 } & 1.0 & 0.189690 & 0.094850 & 0.197710 & \text { Biso } & 1.000000 \mathrm{~S} \\ \text { S17 } & 1.0 & 0.860108 & 0.626173 & 0.342075 & \text { Biso } & 1.000000 \mathrm{~S} \\ \text { S18 } & 1.0 & 0.830550 & 0.612740 & 0.100790 & \text { Biso } & 1.000000 \mathrm{~S} \\ \text { S19 } & 1.0 & 0.614226 & 0.937335 & 0.434393 & \text { Biso } & 1.000000 \mathrm{~S} \\ \text { S20 } & 1.0 & 0.583830 & 0.919330 & 0.186690 & \text { Biso } & 1.000000 \mathrm{~S} \\ \text { S21 } & 1.0 & 0.243925 & 0.799190 & 0.284501 & \text { Biso } & 1.000000 \mathrm{~S} \\ \text { S22 } & 1.0 & 0.219570 & 0.784900 & 0.038280 & \text { Biso } & 1.000000 \mathrm{~S} \\ \text { S23 } & 1.0 & 0.127361 & 0.865390 & 0.409756 & \text { Biso } & 1.000000 \mathrm{~S} \\ \text { S24 } & 1.0 & 0.099960 & 0.852520 & 0.156260 & \text { Biso } & 1.000000 \mathrm{~S} \\ \text { S25 } & 1.0 & 0.373150 & 0.557475 & 0.316626 & \text { Biso } & 1.000000 \mathrm{~S} \\ \text { S26 } & 1.0 & 0.346680 & 0.545920 & 0.070350 & \text { Biso } & 1.000000 \mathrm{~S} \\ \text { S27 } & 1.0 & 0.745609 & 0.703141 & 0.471051 & \text { Biso } & 1.000000 \mathrm{~S} \\ \text { S28 } & 1.0 & 0.710930 & 0.680350 & 0.218760 & \text { Biso } & 1.000000 \mathrm{~S} \\ \text { S29 } & 1.0 & 0.765619 & 0.881108 & 0.306188 & \text { Biso } & 1.000000 \mathrm{~S} \\ \text { S30 } & 1.0 & 0.740810 & 0.870400 & 0.059330 & \text { Biso } & 1.000000 \mathrm{~S} \\ \text { S31 } & 1.0 & 0.210117 & 0.618237 & 0.448042 & \text { Biso } & 1.000000 \mathrm{~S} \\ \text { S32 } & 1.0 & 0.189690 & 0.594850 & 0.197710 & \text { Biso } & 1.000000 \mathrm{~S}\end{array}$

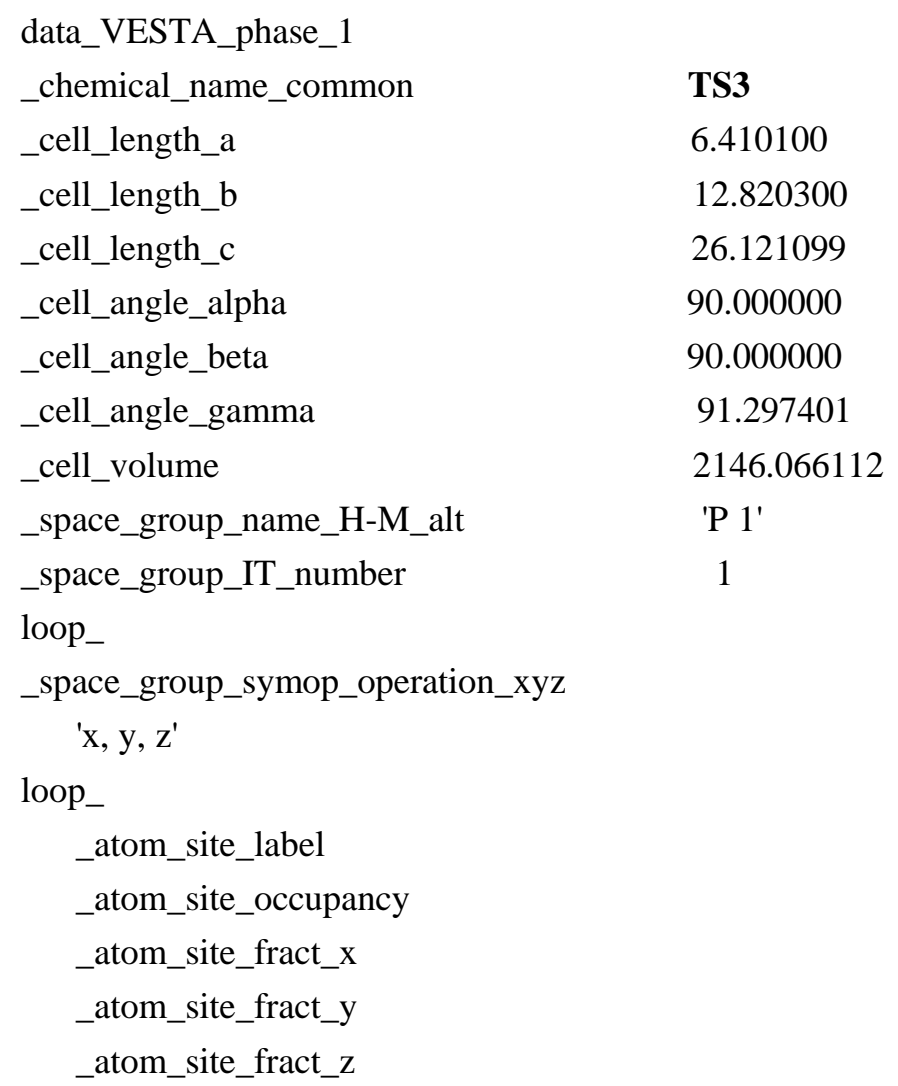

TS3

6.410100

12.820300

26.121099

90.000000

90.000000

91.297401

2146.066112

'P 1'

1 


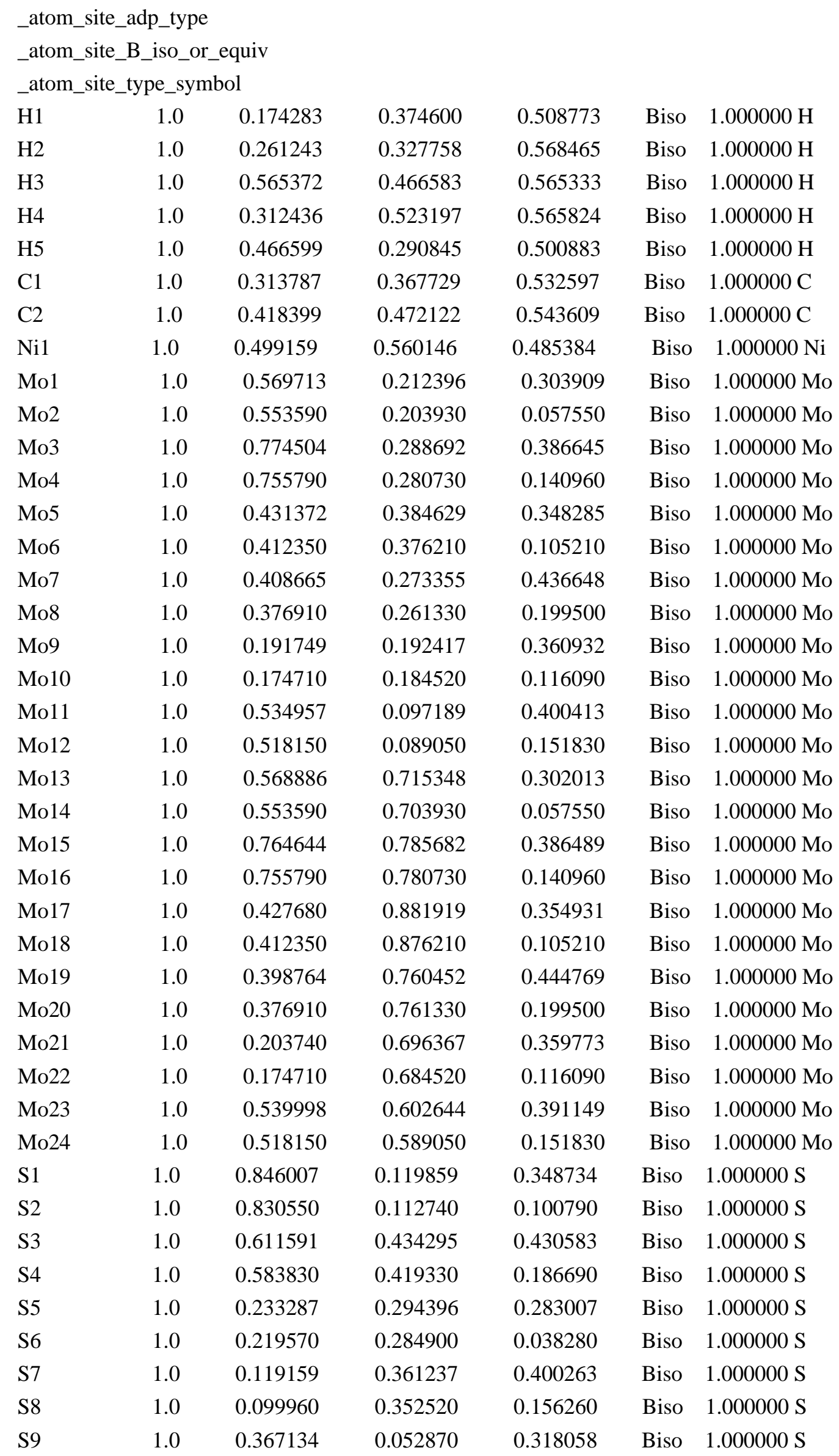




$\begin{array}{lllllll}\text { S10 } & 1.0 & 0.346680 & 0.045920 & 0.070350 & \text { Biso } & 1.000000 \mathrm{~S} \\ \text { S11 } & 1.0 & 0.732221 & 0.190577 & 0.466119 & \text { Biso } & 1.000000 \mathrm{~S} \\ \text { S12 } & 1.0 & 0.710930 & 0.180350 & 0.218760 & \text { Biso } & 1.000000 \mathrm{~S} \\ \text { S13 } & 1.0 & 0.761979 & 0.380719 & 0.305408 & \text { Biso } & 1.000000 \mathrm{~S} \\ \text { S14 } & 1.0 & 0.740810 & 0.370400 & 0.059330 & \text { Biso } & 1.000000 \mathrm{~S} \\ \text { S15 } & 1.0 & 0.208717 & 0.110685 & 0.444930 & \text { Biso } & 1.000000 \mathrm{~S} \\ \text { S16 } & 1.0 & 0.189690 & 0.094850 & 0.197710 & \text { Biso } & 1.000000 \mathrm{~S} \\ \text { S17 } & 1.0 & 0.852650 & 0.622039 & 0.341163 & \text { Biso } & 1.000000 \mathrm{~S} \\ \text { S18 } & 1.0 & 0.830550 & 0.612740 & 0.100790 & \text { Biso } & 1.000000 \mathrm{~S} \\ \text { S19 } & 1.0 & 0.594351 & 0.925656 & 0.436817 & \text { Biso } & 1.000000 \mathrm{~S} \\ \text { S20 } & 1.0 & 0.583830 & 0.919330 & 0.186690 & \text { Biso } & 1.000000 \mathrm{~S} \\ \text { S21 } & 1.0 & 0.237436 & 0.799680 & 0.283884 & \text { Biso } & 1.000000 \mathrm{~S} \\ \text { S22 } & 1.0 & 0.219570 & 0.784900 & 0.038280 & \text { Biso } & 1.000000 \mathrm{~S} \\ \text { S23 } & 1.0 & 0.114293 & 0.858859 & 0.408112 & \text { Biso } & 1.000000 \mathrm{~S} \\ \text { S24 } & 1.0 & 0.099960 & 0.852520 & 0.156260 & \text { Biso } & 1.000000 \mathrm{~S} \\ \text { S25 } & 1.0 & 0.359144 & 0.555461 & 0.312029 & \text { Biso } & 1.000000 \mathrm{~S} \\ \text { S26 } & 1.0 & 0.346680 & 0.545920 & 0.070350 & \text { Biso } & 1.000000 \mathrm{~S} \\ \text { S27 } & 1.0 & 0.728105 & 0.688925 & 0.468179 & \text { Biso } & 1.000000 \mathrm{~S} \\ \text { S28 } & 1.0 & 0.710930 & 0.680350 & 0.218760 & \text { Biso } & 1.000000 \mathrm{~S} \\ \text { S29 } & 1.0 & 0.753992 & 0.880747 & 0.306975 & \text { Biso } & 1.000000 \mathrm{~S} \\ \text { S30 } & 1.0 & 0.740810 & 0.870400 & 0.059330 & \text { Biso } & 1.000000 \mathrm{~S} \\ \text { S31 } & 1.0 & 0.202463 & 0.602554 & 0.445976 & \text { Biso } & 1.000000 \mathrm{~S} \\ \text { S32 } & 1.0 & 0.189690 & 0.594850 & 0.197710 & \text { Biso } & 1.000000 \mathrm{~S}\end{array}$

data_VESTA_phase_1

\begin{tabular}{|c|c|}
\hline _chemical_name_common & $\mathrm{C}_{2} \mathrm{H}_{5} *-\mathrm{Ni}_{1} \mathrm{MoS}$ \\
\hline _cell_length_a & 6.410100 \\
\hline _cell_length_b & 12.820300 \\
\hline _cell_length_c & 26.121099 \\
\hline _cell_angle_alpha & 90.000000 \\
\hline _cell_angle_beta & 90.000000 \\
\hline _cell_angle_gamma & 91.297401 \\
\hline _cell_volume & 2146.066112 \\
\hline _space_group_name_H-M_alt & 'P 1' \\
\hline _space_group_IT_number & 1 \\
\hline loop $_{-}$ & \\
\hline _space_group_symop_operation_xyz & \\
\hline 'x, y, z' & \\
\hline loop_ & \\
\hline _atom_site_label & \\
\hline _atom_site_occupancy & \\
\hline _atom_site_fract_x & \\
\hline _atom_site_fract_y & \\
\hline
\end{tabular}




\begin{tabular}{|c|c|c|c|c|c|c|}
\hline \multicolumn{7}{|c|}{$\begin{array}{l}\text { _atom_site_adp_type } \\
\text { _atom_site_B_iso_or_equiv } \\
\text { _atom_site_type_symbol }\end{array}$} \\
\hline $\mathrm{H} 1$ & 1.0 & 0.176170 & 0.373812 & 0.509116 & Biso & $1.000000 \mathrm{H}$ \\
\hline $\mathrm{H} 2$ & 1.0 & 0.250868 & 0.331907 & 0.570557 & Biso & $1.000000 \mathrm{H}$ \\
\hline $\mathrm{H} 3$ & 1.0 & 0.565026 & 0.467246 & 0.565276 & Biso & $1.000000 \mathrm{H}$ \\
\hline $\mathrm{H} 4$ & 1.0 & 0.312276 & 0.525542 & 0.566521 & Biso & $1.000000 \mathrm{H}$ \\
\hline H5 & 1.0 & 0.427263 & 0.311523 & 0.521102 & Biso & $1.000000 \mathrm{H}$ \\
\hline $\mathrm{C} 1$ & 1.0 & 0.310608 & 0.369056 & 0.535140 & Biso & $1.000000 \mathrm{C}$ \\
\hline $\mathrm{C} 2$ & 1.0 & 0.415655 & 0.474366 & 0.544694 & Biso & $1.000000 \mathrm{C}$ \\
\hline Ni1 & 1.0 & 0.498869 & 0.559724 & 0.485474 & Biso & $1.000000 \mathrm{Ni}$ \\
\hline Mo1 & 1.0 & 0.569981 & 0.212335 & 0.303918 & Biso & $1.000000 \mathrm{Mo}$ \\
\hline Mo2 & 1.0 & 0.553590 & 0.203930 & 0.057550 & Biso & $1.000000 \mathrm{Mo}$ \\
\hline Mo3 & 1.0 & 0.774516 & 0.288566 & 0.386928 & Biso & $1.000000 \mathrm{Mo}$ \\
\hline Mo4 & 1.0 & 0.755790 & 0.280730 & 0.140960 & Biso & $1.000000 \mathrm{Mo}$ \\
\hline Mo5 & 1.0 & 0.430944 & 0.383826 & 0.348826 & Biso & $1.000000 \mathrm{Mo}$ \\
\hline Mo6 & 1.0 & 0.412350 & 0.376210 & 0.105210 & Biso & $1.000000 \mathrm{Mo}$ \\
\hline Mo7 & 1.0 & 0.410427 & 0.272918 & 0.439307 & Biso & $1.000000 \mathrm{Mo}$ \\
\hline Mo8 & 1.0 & 0.376910 & 0.261330 & 0.199500 & Biso & $1.000000 \mathrm{Mo}$ \\
\hline Mo9 & 1.0 & 0.193569 & 0.193548 & 0.361587 & Biso & $1.000000 \mathrm{Mo}$ \\
\hline Mo10 & 1.0 & 0.174710 & 0.184520 & 0.116090 & Biso & $1.000000 \mathrm{Mo}$ \\
\hline Mo11 & 1.0 & 0.535441 & 0.098222 & 0.400855 & Biso & $1.000000 \mathrm{Mo}$ \\
\hline Mo12 & 1.0 & 0.518150 & 0.089050 & 0.151830 & Biso & $1.000000 \mathrm{Mo}$ \\
\hline Mo13 & 1.0 & 0.568727 & 0.715238 & 0.302018 & Biso & $1.000000 \mathrm{Mo}$ \\
\hline Mo14 & 1.0 & 0.553590 & 0.703930 & 0.057550 & Biso & $1.000000 \mathrm{Mo}$ \\
\hline Mo15 & 1.0 & 0.764693 & 0.785750 & 0.386487 & Biso & $1.000000 \mathrm{Mo}$ \\
\hline Mo16 & 1.0 & 0.755790 & 0.780730 & 0.140960 & Biso & $1.000000 \mathrm{Mo}$ \\
\hline Mo17 & 1.0 & 0.427711 & 0.882019 & 0.354921 & Biso & $1.000000 \mathrm{Mo}$ \\
\hline Mo18 & 1.0 & 0.412350 & 0.876210 & 0.105210 & Biso & $1.000000 \mathrm{Mo}$ \\
\hline Mo19 & 1.0 & 0.398845 & 0.760271 & 0.444849 & Biso & $1.000000 \mathrm{Mo}$ \\
\hline Mo20 & 1.0 & 0.376910 & 0.761330 & 0.199500 & Biso & $1.000000 \mathrm{Mo}$ \\
\hline Mo21 & 1.0 & 0.203570 & 0.696284 & 0.359779 & Biso & $1.000000 \mathrm{Mo}$ \\
\hline Mo22 & 1.0 & 0.174710 & 0.684520 & 0.116090 & Biso & $1.000000 \mathrm{Mo}$ \\
\hline Mo23 & 1.0 & 0.540059 & 0.602558 & 0.391188 & Biso & $1.000000 \mathrm{Mo}$ \\
\hline Mo24 & 1.0 & 0.518150 & 0.589050 & 0.151830 & Biso & $1.000000 \mathrm{Mo}$ \\
\hline $\mathrm{S} 1$ & 1.0 & 0.846472 & 0.120141 & 0.348808 & Biso & $1.000000 \mathrm{~S}$ \\
\hline $\mathrm{S} 2$ & 1.0 & 0.830550 & 0.112740 & 0.100790 & Biso & $1.000000 \mathrm{~S}$ \\
\hline S3 & 1.0 & 0.611305 & 0.434047 & 0.430880 & Biso & $1.000000 \mathrm{~S}$ \\
\hline S4 & 1.0 & 0.583830 & 0.419330 & 0.186690 & Biso & $1.000000 \mathrm{~S}$ \\
\hline S5 & 1.0 & 0.233719 & 0.294381 & 0.283165 & Biso & $1.000000 \mathrm{~S}$ \\
\hline S6 & 1.0 & 0.219570 & 0.284900 & 0.038280 & Biso & $1.000000 \mathrm{~S}$ \\
\hline S7 & 1.0 & 0.120203 & 0.360806 & 0.401113 & Biso & $1.000000 \mathrm{~S}$ \\
\hline S8 & 1.0 & 0.099960 & 0.352520 & 0.156260 & Biso & $1.000000 \mathrm{~S}$ \\
\hline
\end{tabular}




$\begin{array}{lllllll}\text { S9 } & 1.0 & 0.367235 & 0.053138 & 0.318298 & \text { Biso } & 1.000000 \mathrm{~S} \\ \text { S10 } & 1.0 & 0.346680 & 0.045920 & 0.070350 & \text { Biso } & 1.000000 \mathrm{~S} \\ \text { S11 } & 1.0 & 0.733524 & 0.190640 & 0.466777 & \text { Biso } & 1.000000 \mathrm{~S} \\ \text { S12 } & 1.0 & 0.710930 & 0.180350 & 0.218760 & \text { Biso } & 1.000000 \mathrm{~S} \\ \text { S13 } & 1.0 & 0.761781 & 0.380471 & 0.305576 & \text { Biso } & 1.000000 \mathrm{~S} \\ \text { S14 } & 1.0 & 0.740810 & 0.370400 & 0.059330 & \text { Biso } & 1.000000 \mathrm{~S} \\ \text { S15 } & 1.0 & 0.208799 & 0.110794 & 0.445171 & \text { Biso } & 1.000000 \mathrm{~S} \\ \text { S16 } & 1.0 & 0.189690 & 0.094850 & 0.197710 & \text { Biso } & 1.000000 \mathrm{~S} \\ \text { S17 } & 1.0 & 0.852502 & 0.622116 & 0.341155 & \text { Biso } & 1.000000 \mathrm{~S} \\ \text { S18 } & 1.0 & 0.830550 & 0.612740 & 0.100790 & \text { Biso } & 1.000000 \mathrm{~S} \\ \text { S19 } & 1.0 & 0.594350 & 0.925774 & 0.436656 & \text { Biso } & 1.000000 \mathrm{~S} \\ \text { S20 } & 1.0 & 0.583830 & 0.919330 & 0.186690 & \text { Biso } & 1.000000 \mathrm{~S} \\ \text { S21 } & 1.0 & 0.237469 & 0.799646 & 0.283872 & \text { Biso } & 1.000000 \mathrm{~S} \\ \text { S22 } & 1.0 & 0.219570 & 0.784900 & 0.038280 & \text { Biso } & 1.000000 \mathrm{~S} \\ \text { S23 } & 1.0 & 0.114486 & 0.858799 & 0.408106 & \text { Biso } & 1.000000 \mathrm{~S} \\ \text { S24 } & 1.0 & 0.099960 & 0.852520 & 0.156260 & \text { Biso } & 1.000000 \mathrm{~S} \\ \text { S25 } & 1.0 & 0.359298 & 0.555232 & 0.312141 & \text { Biso } & 1.000000 \mathrm{~S} \\ \text { S26 } & 1.0 & 0.346680 & 0.545920 & 0.070350 & \text { Biso } & 1.000000 \mathrm{~S} \\ \text { S27 } & 1.0 & 0.728047 & 0.688828 & 0.468195 & \text { Biso } & 1.000000 \mathrm{~S} \\ \text { S28 } & 1.0 & 0.710930 & 0.680350 & 0.218760 & \text { Biso } & 1.000000 \mathrm{~S} \\ \text { S29 } & 1.0 & 0.754093 & 0.880709 & 0.306975 & \text { Biso } & 1.000000 \mathrm{~S} \\ \text { S30 } & 1.0 & 0.740810 & 0.870400 & 0.059330 & \text { Biso } & 1.000000 \mathrm{~S} \\ \text { S31 } & 1.0 & 0.202419 & 0.602493 & 0.446000 & \text { Biso } & 1.000000 \mathrm{~S} \\ \text { S32 } & 1.0 & 0.189690 & 0.594850 & 0.197710 & \text { Biso } & 1.000000 \mathrm{~S}\end{array}$

data_VESTA_phase_1

_chemical_name_common

TS4

_cell_length_a

6.410100

12.820300

_cell_length_b

26.121099

_cell_length_c

90.000000

_cell_angle_beta

90.000000

91.297401

_cell_angle_gamma

2146.066112

_cell_volume

'P 1'

_space_group_name_H-M_alt

1

loop_

_space_group_symop_operation_xyz

'x, y, z'

loop_

_atom_site_label

_atom_site_occupancy

_atom_site_fract_x 


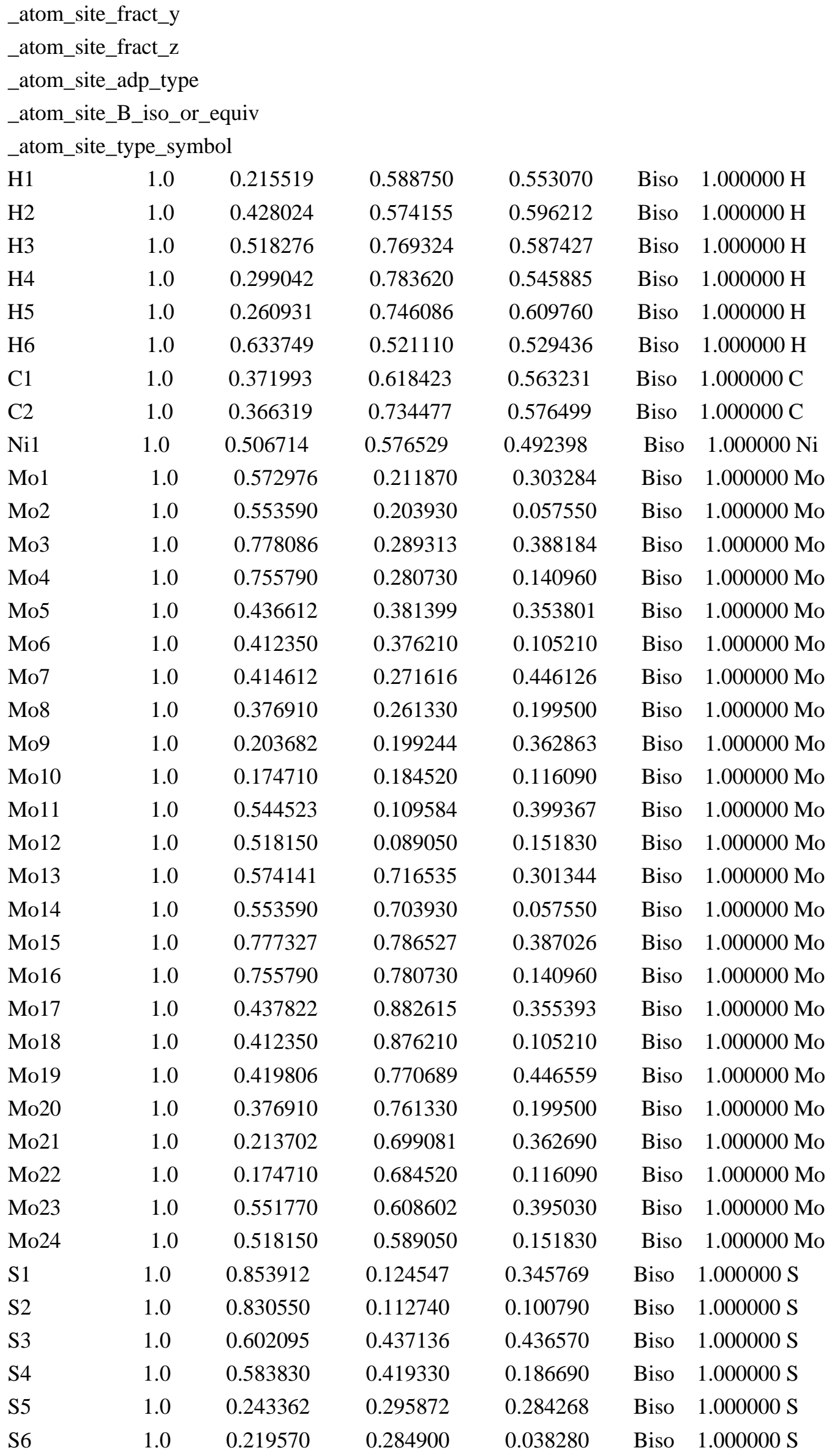




$\begin{array}{lllllll}\text { S7 } & 1.0 & 0.123739 & 0.363439 & 0.408413 & \text { Biso } 1.000000 \mathrm{~S} \\ \text { S8 } & 1.0 & 0.099960 & 0.352520 & 0.156260 & \text { Biso } & 1.000000 \mathrm{~S} \\ \text { S9 } & 1.0 & 0.364031 & 0.054524 & 0.318706 & \text { Biso } & 1.000000 \mathrm{~S} \\ \text { S10 } & 1.0 & 0.346680 & 0.045920 & 0.070350 & \text { Biso } & 1.000000 \mathrm{~S} \\ \text { S11 } & 1.0 & 0.737036 & 0.201395 & 0.470409 & \text { Biso } & 1.000000 \mathrm{~S} \\ \text { S12 } & 1.0 & 0.710930 & 0.180350 & 0.218760 & \text { Biso } & 1.000000 \mathrm{~S} \\ \text { S13 } & 1.0 & 0.766559 & 0.375197 & 0.306136 & \text { Biso } & 1.000000 \mathrm{~S} \\ \text { S14 } & 1.0 & 0.740810 & 0.370400 & 0.059330 & \text { Biso } & 1.000000 \mathrm{~S} \\ \text { S15 } & 1.0 & 0.207616 & 0.117275 & 0.446107 & \text { Biso } & 1.000000 \mathrm{~S} \\ \text { S16 } & 1.0 & 0.189690 & 0.094850 & 0.197710 & \text { Biso } 1.000000 \mathrm{~S} \\ \text { S17 } & 1.0 & 0.858901 & 0.623732 & 0.341655 & \text { Biso } & 1.000000 \mathrm{~S} \\ \text { S18 } & 1.0 & 0.830550 & 0.612740 & 0.100790 & \text { Biso } & 1.000000 \mathrm{~S} \\ \text { S19 } & 1.0 & 0.614283 & 0.934622 & 0.434370 & \text { Biso } & 1.000000 \mathrm{~S} \\ \text { S20 } & 1.0 & 0.583830 & 0.919330 & 0.186690 & \text { Biso } & 1.000000 \mathrm{~S} \\ \text { S21 } & 1.0 & 0.243948 & 0.797304 & 0.284557 & \text { Biso } & 1.000000 \mathrm{~S} \\ \text { S22 } & 1.0 & 0.219570 & 0.784900 & 0.038280 & \text { Biso } & 1.000000 \mathrm{~S} \\ \text { S23 } & 1.0 & 0.125888 & 0.862158 & 0.410074 & \text { Biso } & 1.000000 \mathrm{~S} \\ \text { S24 } & 1.0 & 0.099960 & 0.852520 & 0.156260 & \text { Biso } & 1.000000 \mathrm{~S} \\ \text { S25 } & 1.0 & 0.371805 & 0.554885 & 0.316478 & \text { Biso } & 1.000000 \mathrm{~S} \\ \text { S26 } & 1.0 & 0.346680 & 0.545920 & 0.070350 & \text { Biso } & 1.000000 \mathrm{~S} \\ \text { S27 } & 1.0 & 0.743571 & 0.702912 & 0.471499 & \text { Biso } 1.000000 \mathrm{~S} \\ \text { S28 } & 1.0 & 0.710930 & 0.680350 & 0.218760 & \text { Biso } 1.000000 \mathrm{~S} \\ \text { S29 } & 1.0 & 0.764928 & 0.879505 & 0.306726 & \text { Biso } & 1.000000 \mathrm{~S} \\ \text { S30 } & 1.0 & 0.740810 & 0.870400 & 0.059330 & \text { Biso } & 1.000000 \mathrm{~S} \\ \text { S31 } & 1.0 & 0.208584 & 0.615147 & 0.447129 & \text { Biso } & 1.000000 \mathrm{~S} \\ \text { S32 } & 1.0 & 0.189690 & 0.594850 & 0.197710 & \text { Biso } 1.000000 \mathrm{~S}\end{array}$

data_VESTA_phase_1

_chemical_name_common

$\mathrm{C}_{2} \mathrm{H}_{6} *{ }^{*}-\mathrm{Ni}_{1} \mathrm{MoS}$

6.410100

12.820300

26.121099

90.000000

90.000000

91.297401

2146.066112

'P 1'

1

_space_group_IT_number

loop_

_space_group_symop_operation_xyz

'x, y, z'

loop_

_atom_site_label 


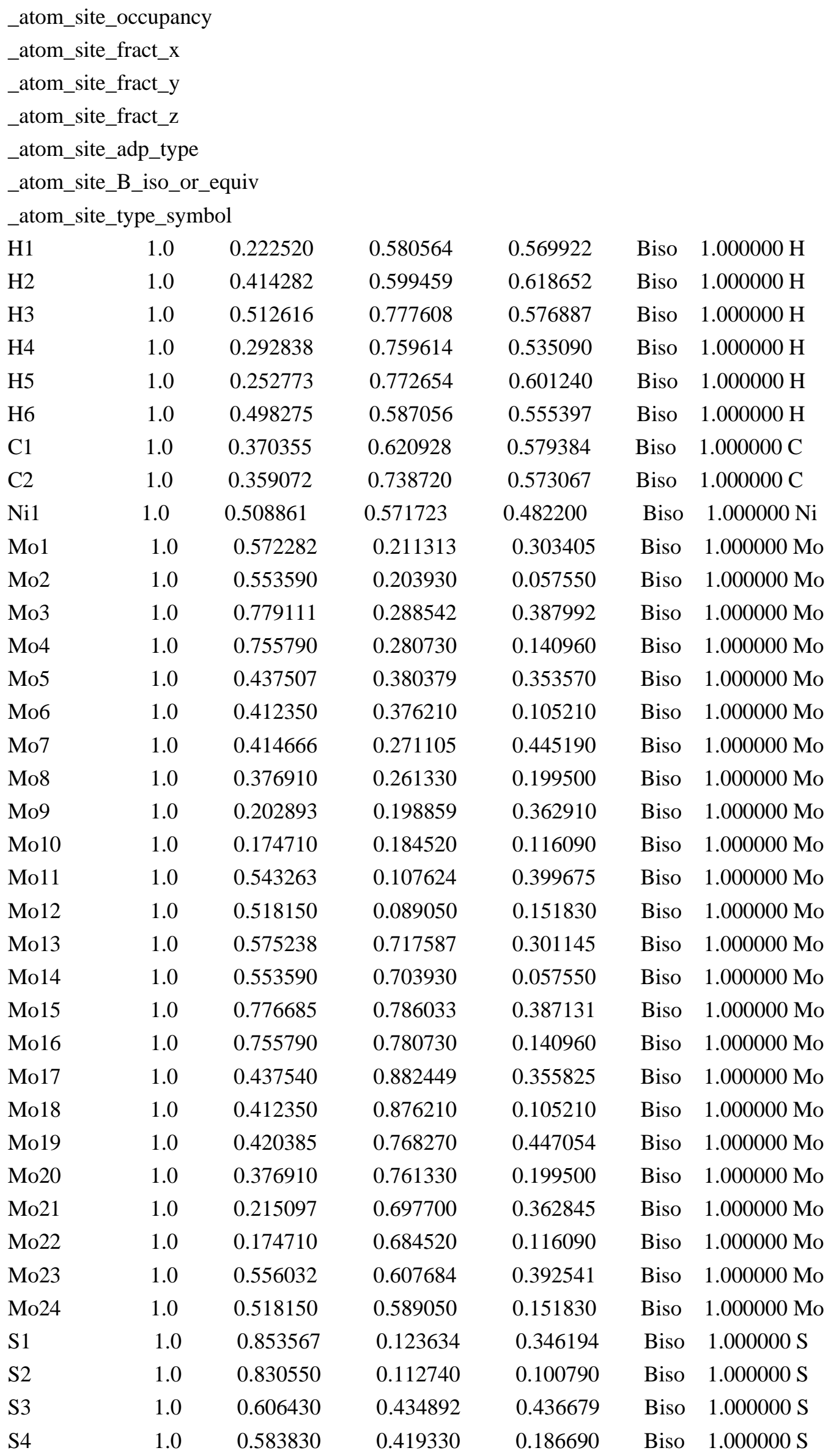




$\begin{array}{lllllll}\text { S5 } & 1.0 & 0.242405 & 0.295840 & 0.284333 & \text { Biso } & 1.000000 \mathrm{~S} \\ \text { S6 } & 1.0 & 0.219570 & 0.284900 & 0.038280 & \text { Biso } & 1.000000 \mathrm{~S} \\ \text { S7 } & 1.0 & 0.124114 & 0.363341 & 0.407938 & \text { Biso } & 1.000000 \mathrm{~S} \\ \text { S8 } & 1.0 & 0.099960 & 0.352520 & 0.156260 & \text { Biso } & 1.000000 \mathrm{~S} \\ \text { S9 } & 1.0 & 0.364286 & 0.053929 & 0.318879 & \text { Biso } & 1.000000 \mathrm{~S} \\ \text { S10 } & 1.0 & 0.346680 & 0.045920 & 0.070350 & \text { Biso } & 1.000000 \mathrm{~S} \\ \text { S11 } & 1.0 & 0.736602 & 0.199234 & 0.470140 & \text { Biso } & 1.000000 \mathrm{~S} \\ \text { S12 } & 1.0 & 0.710930 & 0.180350 & 0.218760 & \text { Biso } & 1.000000 \mathrm{~S} \\ \text { S13 } & 1.0 & 0.767368 & 0.374433 & 0.305970 & \text { Biso } & 1.000000 \mathrm{~S} \\ \text { S14 } & 1.0 & 0.740810 & 0.370400 & 0.059330 & \text { Biso } & 1.000000 \mathrm{~S} \\ \text { S15 } & 1.0 & 0.208610 & 0.115830 & 0.446295 & \text { Biso } & 1.000000 \mathrm{~S} \\ \text { S16 } & 1.0 & 0.189690 & 0.094850 & 0.197710 & \text { Biso } & 1.000000 \mathrm{~S} \\ \text { S17 } & 1.0 & 0.861069 & 0.623773 & 0.340655 & \text { Biso } & 1.000000 \mathrm{~S} \\ \text { S18 } & 1.0 & 0.830550 & 0.612740 & 0.100790 & \text { Biso } & 1.000000 \mathrm{~S} \\ \text { S19 } & 1.0 & 0.613837 & 0.933285 & 0.434951 & \text { Biso } & 1.000000 \mathrm{~S} \\ \text { S20 } & 1.0 & 0.583830 & 0.919330 & 0.186690 & \text { Biso } & 1.000000 \mathrm{~S} \\ \text { S21 } & 1.0 & 0.244107 & 0.796512 & 0.284872 & \text { Biso } & 1.000000 \mathrm{~S} \\ \text { S22 } & 1.0 & 0.219570 & 0.784900 & 0.038280 & \text { Biso } & 1.000000 \mathrm{~S} \\ \text { S23 } & 1.0 & 0.126666 & 0.860950 & 0.410389 & \text { Biso } & 1.000000 \mathrm{~S} \\ \text { S24 } & 1.0 & 0.099960 & 0.852520 & 0.156260 & \text { Biso } & 1.000000 \mathrm{~S} \\ \text { S25 } & 1.0 & 0.372405 & 0.554446 & 0.315554 & \text { Biso } & 1.000000 \mathrm{~S} \\ \text { S26 } & 1.0 & 0.346680 & 0.545920 & 0.070350 & \text { Biso } & 1.000000 \mathrm{~S} \\ \text { S27 } & 1.0 & 0.746696 & 0.699155 & 0.471878 & \text { Biso } & 1.000000 \mathrm{~S} \\ \text { S28 } & 1.0 & 0.710930 & 0.680350 & 0.218760 & \text { Biso } & 1.000000 \mathrm{~S} \\ \text { S29 } & 1.0 & 0.765106 & 0.879987 & 0.307085 & \text { Biso } & 1.000000 \mathrm{~S} \\ \text { S30 } & 1.0 & 0.740810 & 0.870400 & 0.059330 & \text { Biso } & 1.000000 \mathrm{~S} \\ \text { S31 } & 1.0 & 0.205356 & 0.615086 & 0.447766 & \text { Biso } & 1.000000 \mathrm{~S} \\ \text { S32 } & 1.0 & 0.189690 & 0.594850 & 0.197710 & \text { Biso } & 1.000000 \mathrm{~S}\end{array}$


To confirm the constructed computational model in well agreement with the experimental finding, Bader charge analysis was firstly carried out to give the electronic state of $\mathrm{Ni}$ species in $\mathrm{Ni}{ }_{1} \mathrm{MoS} / \mathrm{Al}_{2} \mathrm{O}_{3}$. As expected, the number of valence charge for $\mathrm{Ni}$ species in $\mathrm{Ni}_{1} \mathrm{MoS} / \mathrm{Al}_{2} \mathrm{O}_{3}$ (Fig. S22) is 9.6, which is smaller than that for neutral $\mathrm{Ni}$ atom (10) but higher than for $\mathrm{Ni}^{2+}(8)$, illustrating that the oxidation state of $\mathrm{Ni}$ species in $\mathrm{Ni}_{1} \mathrm{MoS} / \mathrm{Al}_{2} \mathrm{O}_{3}$ is positive but below 2 (namely $\mathrm{Ni}^{\delta+}$ species where $\delta$ lies in the range $0-2)$.

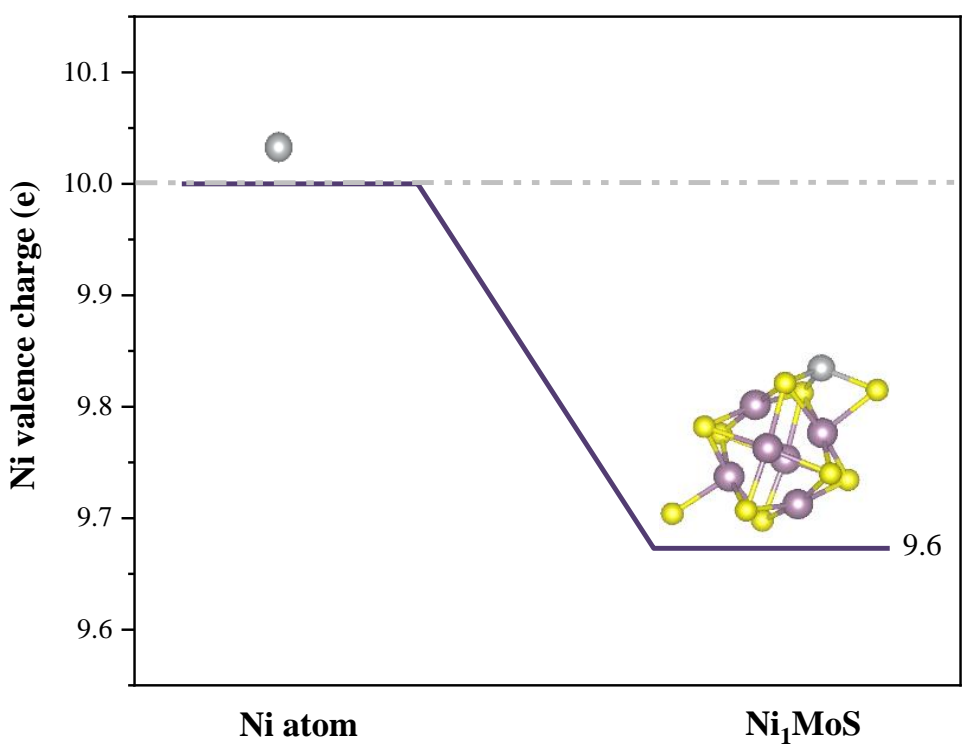

Fig. S22 Ni valence charge of $\mathrm{Ni}_{1} \mathrm{MoS} / \mathrm{Al}_{2} \mathrm{O}_{3}$ and neutral $\mathrm{Ni}$ atom. The insets show the structures of the corresponding model.

To achieve a better understanding on the effects of single $\mathrm{Ni}$ atoms in $\mathrm{Ni}{ }_{1} \mathrm{MoS} / \mathrm{Al}_{2} \mathrm{O}_{3}$ catalyst on the ethylene selectivity, $\mathrm{C}_{2} \mathrm{H}_{4}$-TPD measurement was conducted to investigate the desorption characteristics of the ethylene product on $\mathrm{Ni}_{1} \mathrm{MoS} / \mathrm{Al}_{2} \mathrm{O}_{3}$ and $\mathrm{Ni} / \mathrm{Al}_{2} \mathrm{O}_{3}$ catalysts. As shown in Fig. S23, three main desorption peaks are observed over $\mathrm{Ni} / \mathrm{Al}_{2} \mathrm{O}_{3}$ catalyst. The first two peaks (at $220-350{ }^{\circ} \mathrm{C}$ ) are ascribed to bridge- and multi-coordinated ethylene species, which are easier to generate the ethane. ${ }^{25}$ Peak at ca. $400{ }^{\circ} \mathrm{C}$ originates from desorption of ethylene from support. ${ }^{26}$ Notably, different from monometallic $\mathrm{Ni}$ sample, $\mathrm{Ni}{ }_{1} \mathrm{MoS} / \mathrm{Al}_{2} \mathrm{O}_{3}$ catalyst exhibits the dominant desorption peak at lower temperature $\left(158.1^{\circ} \mathrm{C}\right)$, ascribed to the relatively weak ethylene adsorption on top sites, ${ }^{27}$ suggesting that ethylene is more easily desorbed from the 
surface of $\mathrm{Ni}_{1} \mathrm{MoS} / \mathrm{Al}_{2} \mathrm{O}_{3}$. These results demonstrates why exceptional ethylene selectivity is observed.

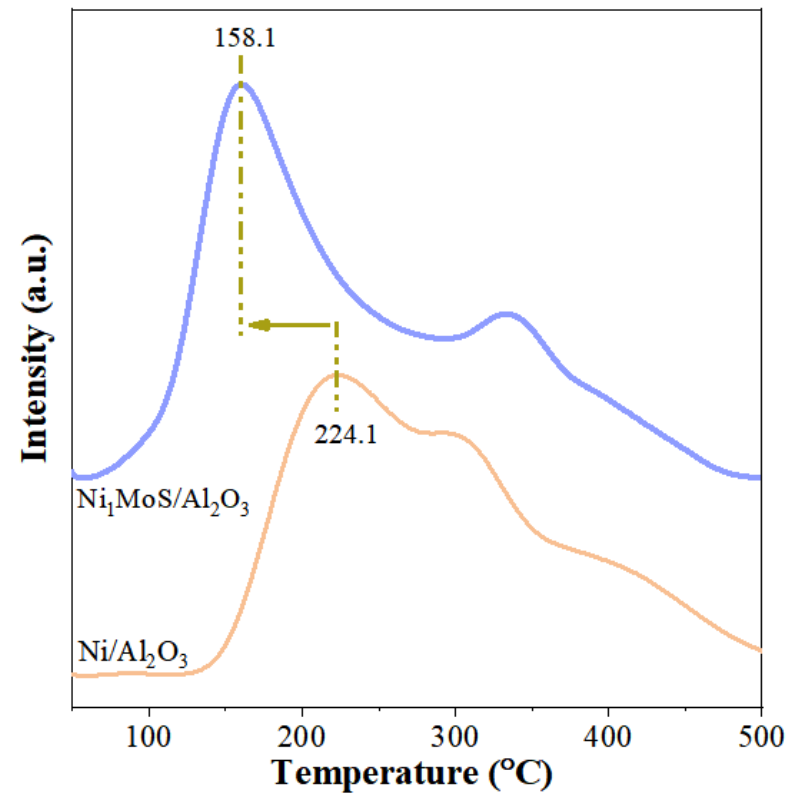

Fig. S23 $\mathrm{C}_{2} \mathrm{H}_{4}-\mathrm{TPD}$ profiles of $\mathrm{Ni}_{1} \mathrm{MoS} / \mathrm{Al}_{2} \mathrm{O}_{3}$ and $\mathrm{Ni} / \mathrm{Al}_{2} \mathrm{O}_{3}$ catalysts

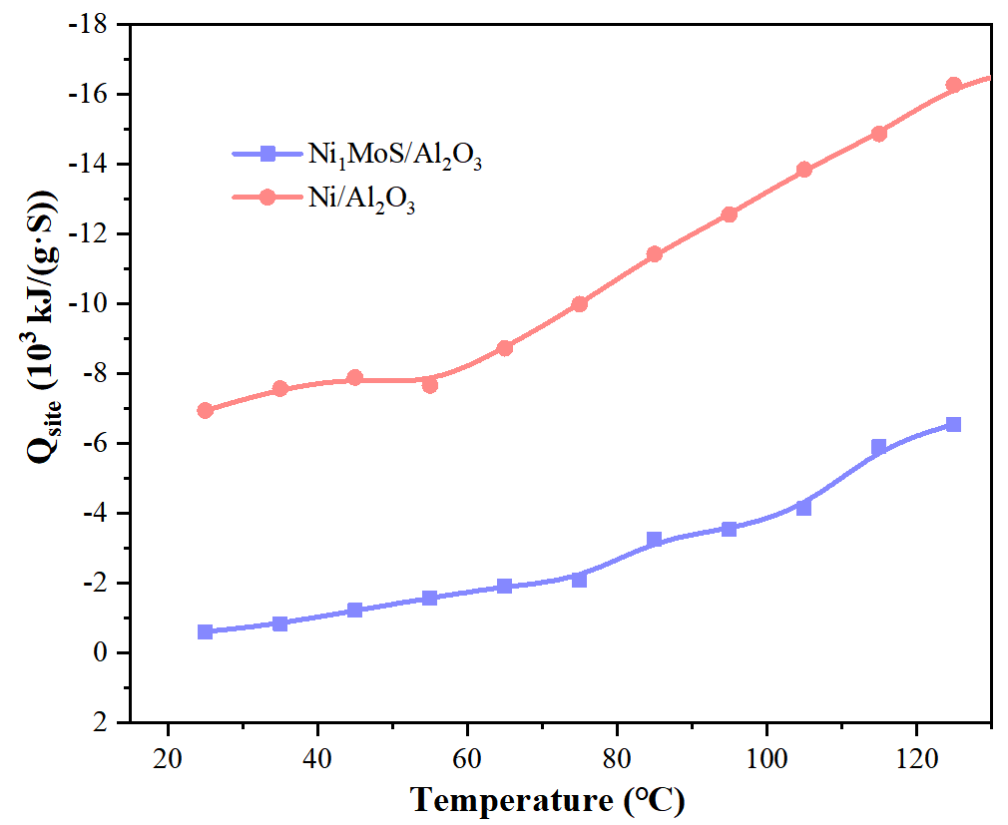

Fig. S24 The exothermic rate of $\mathrm{Ni}_{1} \mathrm{MoS} / \mathrm{Al}_{2} \mathrm{O}_{3}$ and $\mathrm{Ni} / \mathrm{Al}_{2} \mathrm{O}_{3}$ catalysts at different reaction temperatures 


\section{References}

1. Kresse, G.; Furthmüller, J. Efficiency of Ab-Initio Total Energy Calculations for Metals and Semiconductors Using a Plane-Wave Basis Set. Comput. Mater. Sci. 1996, 6, 15-50.

2. Kresse, G.; Furthmüller, J. Efficient Iterative Schemes for Ab Initio Total-Energy Calculations Using a Plane-Wave Basis Set. Phys. Rev. B. 1996, 54, 11169-11186.

3. Perdew, J. P.; Burke, K.; Ernzerhof, M. Generalized Gradient Approximation Made Simple. Phys. Rev. Lett. 1996, 77, 3865-3868.

4. Kresse, G.; Joubert, D. From Ultrasoft Pseudopotentials to the Projector Augmented-Wave Method. Phys. Rev. B. 1999, 59, 1758-1775.

5. Blöchl, P. E. Projector Augmented-Wave Method. Phys. Rev. B. 1994, 50, 17953-17979.

6. Grimme, S.; Antony, J.; Ehrlich, S.; Krieg, H. A Consistent and Accurate ab Initio Parametrization of Density Functional Dispersion Correction (DFT-D) for The 94 Elements HPu. J. Chem. Phys. 2010, 132, 154104.

7. Henkelman, G.; Uberuaga, B. P.; Jonsson, H. A Climbing Image Nudged Elastic Band Method for Finding Saddle Points and Minimum Energy Paths. J. Chem. Phys. 2000, 113, 9901.

8. Hernandez-Molina R, S. A. G. Reactions of The Heterometallic Cuboidal Clusters $\mathrm{Mo}_{3} \mathrm{MS}_{4}$ $(\mathrm{M}=\mathrm{Co}, \mathrm{Ni}, \mathrm{Pd}, \mathrm{Cu})$ and $\mathrm{Mo}_{3} \mathrm{NiSe}_{4}$ with $\mathrm{CO}$ : Electron Counts and Kinetic/Thermodynamic Studies with M = Ni, Pd. Coord Chem Rev. 1999, 187, 291-302.

9. Liu, B.; Liu, L.; Chai, Y. M.; Zhao, J. C.; Li, Y. P.; Liu, Y. Q.; Liu, C. G. Highly Active $\mathrm{CoMoS} / \mathrm{Al}_{2} \mathrm{O}_{3}$ Catalysts ex Situ Presulfided with Ammonium Sulfide for Selective Hydrodesulfurization of Fluid Catalytic Cracking Gasoline. Ind. Eng. Chem. Res. 2018, 57, 2041-2049.

10. Ohki, Y.; Hara, R.; Munakata, K.; Tada, M.; Takayama, T.; Sakai, Y.; and Cramer, R. E. Synthesis of $\left[\mathrm{Mo}_{3} \mathrm{~S}_{4}\right]$ Clusters from Half-Sandwich Molybdenum(V) Chlorides and Their Application as Platforms for [ $\left.\mathrm{Mo}_{3} \mathrm{~S}_{4} \mathrm{Fe}\right]$ Cubes. Inorg. Chem. 2019, 58, 5230-5240.

11. Hernandez-Molina, R.; Gonzalez-Platas, J.; Kovalenko, K. A.; Sokolov, M. N.; Virovets, A. V.; Llusar, R.; Vicent, C. Cuboidal $\mathrm{Mo}_{3} \mathrm{~S}_{4}$ and $\mathrm{Mo}_{3} \mathrm{NiS}_{4}$ Complexes Bearing Dithiophosphates and Chiral Carboxylate Ligands: Synthesis, Crystal Structure and Fluxionality. Eur. J. Inorg. Chem. 2011, 5, 683-693.

12. Boudjahem, A. G.; Chettibi, M.; Monteverdi, S.; Bettahar, M. M. Acetylene Hydrogenation Over Ni-Cu Nanoparticles Supported on Silica Prepared by Aqueous Hydrazine Reduction. $J$. Nanosci. Nanotechnol. 2009, 9, 3546-3554.

13. Zhou, S.; Kang, L.; Xu, Z.; et al. Catalytic Performance and Deactivation of Ni/MCM-41 Catalyst in The Hydrogenation of Pure Acetylene to Ethylene. RSC Adv. 2020, 10, 1937-1945.

14. Pei, G. X.; Liu, X, Y.; Wang, A.; Su, Y.; Li, L.; Zhang, T. Selective Hydrogenation of Acetylene in an Ethylene-Rich Stream over Silica Supported Ag-Ni Bimetallic Catalysts. Appl. Catal. A. 2017, 545, 90-96. 
15. Wu, Y.; Dai, X.; Chen, Z.; et al. Single Ni Sites Distributed on N-doped Carbon for Selective Hydrogenation of Acetylene. Chem. Commun. 2017, 53, 11568-11571.

16. Zhou, G. L.; Wang, P.G.; Jiang, Z. X.; Ying, P. L.; Li, C. Selective Hydrogenation of Acetylene over a MoP Catalyst. Chinese J. Catal. 2011, 32, 27-30.

17. Simanullang, W. F.; Ma, J.; Shimizu, K.; Furukawa, S. Silica-Decorated Ni-Zn Alloy as a Highly Active and Selective Catalyst for Acetylene Semihydrogenation. Catal. Sci. Technol. 2021, 11, 4016-4020.

18. Cao, Y. Q.; Zhang, H.; Ji, S.; Sui, Z. J.; Jiang, Z.; Wang, D. S.; Zaera, F.; Zhou, X.G.; Duan, X. Z.; Li, Y. D. Adsorption Site Regulation to Guide Atomic Design of Ni-Ga Catalysts for Acetylene Semi-hydrogenation. Angew. Chem. 2020, 132, 11744 - 11749.

19. Liu, H.; Chai, M. Q.; Pei, G. X.; Liu, X.; Li, L.; Kang, L.; Wang, A.; Zhang, T. Effect of IBMetal on $\mathrm{Ni} / \mathrm{SiO}_{2}$ Catalyst for Selective Hydrogenation of Acetylene. Chinese J. Catal. 2020, 41, 1099-1108.

20. Chen, Y. J.; Chen, J. X. Selective Hydrogenation of Acetylene on $\mathrm{SiO}_{2}$ Supported Ni-In Bimetallic Catalysts: Promotional Effect of In. Appl. Surf. Sci. 2016, 387, 16-27.

21. Riley, C.; Riva, A. D. L.; Zhou, S.; et al. Synthesis of Nickel-Doped Ceria Catalysts for Selective Acetylene Hydrogenation. ChemCatChem. 2019, 11, 1526-1533.

22. Tsukuda, R.; Yamagishi, R.; Kameoka, S.; et al. Ability of Hydrogen Storage $\mathrm{CeNi}_{5-\mathrm{x}} \mathrm{Ga}_{\mathrm{x}}$ and $\mathrm{Mg}_{2} \mathrm{Ni}$ Alloys to Hydrogenate Acetylene. Sci. Technol. Adv. Mater. 2019, 20, 774-758.

23. Liu, Y. N.; Zhao, J. Y.; Feng, J. T.; He, Y. F.; Du, Y. Y.; Li, D. Q. Layered Double HydroxideDerived Ni-Cu Nanoalloy Catalysts for Semi-Hydrogenation of Alkynes: Improvement of Selectivity and Anti-Coking Ability via Alloying of Ni and Cu. J. Catal. 2018, 359, 251-260.

24. Zhang, Y.; Sun, X.; Zhao, Y.; et al. C, N Co-Decorated Alumina-Supported Au Nanoparticles: Enhanced Catalytic Performance for Selective Hydrogenation of Acetylene. Top Catal. 2021, 64, 197-205.

25. Liu, Y. N.; Feng, J. T.; He, Y. F.; Sun, J. H.; Li, D. Q. Partial Hydrogenation of Acetylene over a NiTi-Layered Double Hydroxide Supported PdAg Catalyst. Catal. Sci. Technol. 2015, 5, 1231-1240.

26. Liu, Y. N.; He, Y. F.; Zhou, D. R.; Feng, J. T.; Li, D. Q. Catalytic Performance of Pd-Promoted $\mathrm{Cu}$ Hydrotalcite-Derived Catalysts in Partial Hydrogenation of Acetylene: Effect of $\mathrm{Pd}-\mathrm{Cu}$ Alloy Formation. Catal. Sci. Technol. 2016, 6, 3027-3037.

27. Cao, Y. Q.; Ge, X. H.; Li, Y. R.; Si, R.; Sui, Z. J.; Zhou, J. H.; Duan, X. Z.; Zhou, X. G. Structural and Kinetics Understanding of Support Effects in Pd-Catalyzed SemiHydrogenation of Acetylene. Engineering. 2021, 7, 103-110. 